\title{
REVIEWS
}

\section{Optically Active Polysilanes. Ten Years of Progress and New Polymer Twist for Nanoscience and Nanotechnology}

\author{
Michiya FuJiKI, ${ }^{*, * *}$ Julian Robert KoE, ${ }^{* * * *}$ Ken TeraO, ${ }^{* * * *}$ Takahiro SAto, ${ }^{* * * * * *}$ \\ Akio Teramoto, ${ }^{* * * * * * *}$ and Junji WATANABE ${ }^{* * * * * * * *}$ \\ ${ }^{*}$ CREST-JST, 4-1-8 Hon-cho, Kawaguchi, Saitama 332-0012, Japan \\ ${ }^{* *}$ Advanced Polymer Science Laboratory, Graduate School of Materials Science, Nara Institute of Science and \\ Technology (NAIST), 8916-5 Takayama, Ikoma, Nara 630-0101, Japan \\ ${ }^{* * *}$ Department of Chemistry, International Christian University, 3-10-2 Osawa, Mitaka, Tokyo 181-8585, Japan \\ ${ }^{* * * *}$ Department of Biological and Chemical Engineering, Gunma University, Kiryu, Gunma 376-8515, Japan \\ ${ }^{* * * * *}$ Department of Macromolecular Science, Osaka University, 1-1 Machikaneyama, Toyonaka, \\ Osaka 560-0043, Japan \\ ${ }^{* * * * * *}$ Research Organization of Science and Engineering, Ritsumeikan University, 1-1-1 Nojihigashi, \\ Kusatsu, Shiga 525-8577, Japan \\ ${ }^{* * * * * * *}$ Department of Organic and Polymeric Materials, Tokyo Institute of Technology, O-okayama, Meguro, \\ Tokyo 152-8552, Japan
}

(Received October 31, 2002; Accepted January 17, 2003)

\begin{abstract}
In the family of optically active synthetic polymers, optically active polysilanes, which comprise a helical main chain of silicon-silicon single bonds and chiral and/or achiral side groups, exhibit unique absorption, circular dichroism, and fluorescence spectra around $300-400 \mathrm{~nm}$ due to $\sigma$-conjugation. Since the first brief report of optically active polysilane synthesis in 1992, the field has now widened to include various homo- and copolymers of optically active poly(dialkylsilane)s, poly(dialkoxysilane)s, poly[alkyl(aryl)silane]s, and poly(diarylsilane)s. This review comprehensively covers work on (i) the relationship between side chain structure, (ii) local structure-global shape relationship, (iii) (chir)optical properties, (iv) (semi)quantitative population analysis of right- and left-handed helices based on Kuhn's dissymmetry ratio, (v) several helical cooperativity effects, (vi) molecular imaging, (vii) inversion of screw-sense, (viii) chiroptical switch and memory, (ix) transfer and amplification of molecular chirality to aggregates, (x) cholesteric liquid crystallinity, (xi) helical supramolecular structures, and (xii) latent helicity, as consequences of side group internal interactions and other external stimuli. Such knowledge and understanding may stimulate optically active polymer research in the realm of nanomaterial science and nanotechnology at the sub-nm level as well as traditional polymer science, and may advance these polymers to new functional nanomaterials and thence to the realization of nanodevices in the future.

KEY WORDS Polysilane / Optically Active / Helix / Chiral / Circular Dichroism / Fluorescence / Conformation /
\end{abstract}

The most important molecular building blocks of our life-amino acids and sugars-lead to left-handed and right-handed helical motifs of natural biopolymers such as DNA, polysaccharides, and proteins. Since the beginning of life on the earth, living organisms preferentially exploit $l$-amino acids and $d$-sugars. Optically active substances, therefore, are ubiquitous in our daily lives and become the vital constituents of drug, vitamins, flavors, fragrances, herbicides, and pesticides. Although the origin and scenario of this biomolecular handedness remain a puzzle for chemists, physicists, biologists, and astronomers, ${ }^{1-5}$ it is now widely accepted that our life is the consequence of chemistry and/or physics of homochiral biomolecular substances.

Outside the realm of these biopolymers, the synthesis, characterization, recognition of unique properties, and application of optically active synthetic polymers with single screw sense stiff helical structures ${ }^{6-12}$ have long been of interest in the area of modern polymer science. This is because these synthetic helical polymers may be promising materials in such areas as enantioselective separation, molecular chirality recognition, chiral nematic liquid crystals, chiroptical switch and memory, nonlinear optics, circularly polarized photoand electroluminescence, and so forth. Knowledge and understanding of the structure-property-functionality relationship using chromophoric optically active polymers, hence, might be vital for further advancement in these and other new areas, as well as in main stream polymer science.

In the pioneering works of synthetic helical polymers two or three decades ago, a few polymers were known to predominantly adopt a single screw sense helical structure in solution at room temperature. For example, Goodman synthesized the first optically active polyisocyanate with chiral side chains in $1970 .^{13}$ 


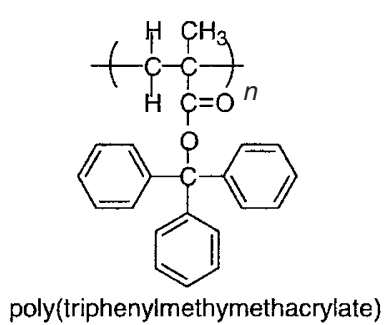

Inherently stable helix with achiral side groups

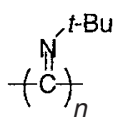

poly(t-butyl isocyanide)

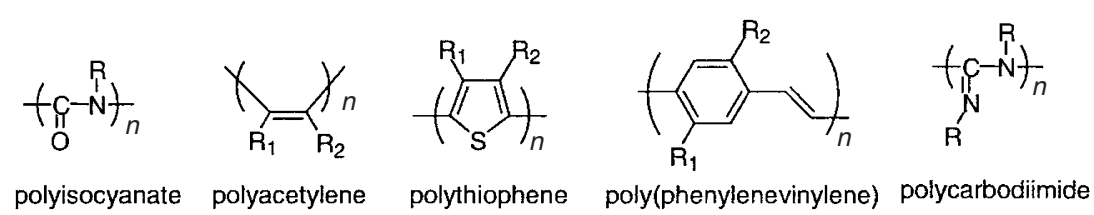

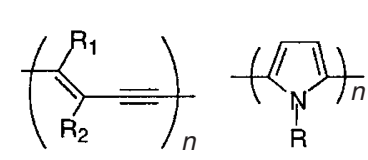

poly(diacetylene)

polypyrrole

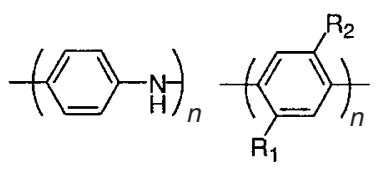

polyaniline

poly ( $p$-phenylene)

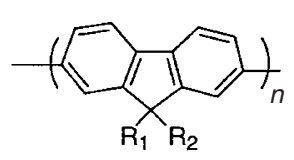

polyfluorene

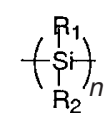

polysilane

Preferential screw sense helix induced by chiral side groups and/or chiral chemical influence

Chart 1. Optically active organic and inorganic polymers with chromophoric main chain and side groups.

Drenth and Nolte in 1974 proved the $4_{1}$ helical structure of poly(t-butyl isocyanide)s which was originally suggested by Millich in $1969 .{ }^{14}$ In 1960, Pino prepared an optically active, isotactic poly- $\alpha$-olefin bearing a chiral side chain, poly(3-methylpentene), followed by optically active poly- $\alpha$-olefin copolymers. ${ }^{15}$ Okamoto and Yuki successfully obtained optically active, isotactic poly(trityl methacrylate) which was the first optically active vinyl polymer to be obtained from a prochiral monomer in $1979 .{ }^{16}$ In those cases, specialized monomers and/or catalysts under controlled polymerization conditions were necessary to produce and retain the helical structures. Since these pioneering studies, many synthetic optically active polymers with $\pi$-conjugating chromophores, including polyisocyanides $,{ }^{8}, 17$ polyisocyanates, ${ }^{9,}, 18$ polyacetylenes, ${ }^{10,19}$ polythiophenes, ${ }^{20}$ poly ( $p$-phenylenevinylene $) \mathrm{s},{ }^{21}$ polycarbodiimides ${ }^{22}$ polydiacetylenes, ${ }^{23}$ polypyrroles, ${ }^{24}$ polyanilines, ${ }^{25}$ poly( $p$-phenylene $) \mathrm{s}^{26}$ polyfluorenes, ${ }^{27}$ and other $\pi$-conjugated polymers and oligomers ${ }^{28}$ have evolved and the research is on-going. Historical aspects and topics of these and other synthetic helical polymers are presented in a recent review article. ${ }^{29}$

Optically active polysilanes, ${ }^{30-35}$ comprising a silicon main chain featuring chiral and/or achiral side groups, are a new class of synthetic chiral polymers. Unique features of these inorganic polymers are the exhibition of intense and sharp UV absorption, circular dichroism (CD), and fluorescence (FL) spectra around $300-400 \mathrm{~nm}$, which are characteristic of the $\sigma$-conjugation in the helical siliconcatenated main chain. ${ }^{35}$ Since the first brief report of the chiroptical properties of poly(dialkylsilane) copolymers with chiral side groups by Matyjaszewski et al. in 1992, ${ }^{30 a}$ various optically active polysilane homo- and copolymers have been studied. ${ }^{30-35}$ Optically active polysilanes constitute a unique nano-structural and multidisciplinary class of polymer. For example, rod-like helical polysilanes may be regarded as ideal model polymers for one-dimensional silicon-based semiconductors or quantum wires with $0.2 \mathrm{~nm}$ width. ${ }^{30 \mathrm{~d}, 30 \mathrm{f}, 36,37}$

This review highlights several significant results characteristic of optically active polysilanes with different degrees of chain stiffness demonstrated by us and other workers, describing the mutual relationship between side chain structure, global conformation and local main chain structures, (chir)optical properties, helical cooperativity, molecular imaging, inversion of screw-sense, chiroptical switch and memory, molecular recognition ability, liquid crystallinity, supramolecular helix, and latent helicity, as consequences of the side group interactions and other external stimuli.

\section{PREPARATION OF OPTICALLY ACTIVE POLYSILANES AND CHARACTERIZATION BY CIRCULAR DICHROISM/FLUORESCENCE/UV ABSORPTION SPECTROSCOPIES}

The synthesis of polysilanes with a high degree of stereogenic control is currently of particular 
<smiles></smiles>
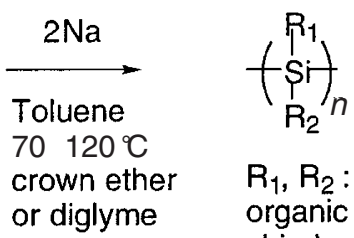

$\mathrm{R}_{1}, \mathrm{R}_{2}$ :

organic group containing chiral groups

Chart 2. Synthesis of optically active polysilanes bearing chiral and/or achiral side groups by Wurtz-type reduction.

interest $\mathrm{s}^{38,39 d-39 f}$ and of significant importance in a wide variety of high performance functions. ${ }^{40}$ Although most optically active polysilanes including poly(dialkylsilane)s, poly(alkylarylsilane)s, and poly(diarylsilane)s are generally prepared by the Wurtz-type condensation of the corresponding dichlorodiorganosilane with sodium in inert solvent, ${ }^{35,39}$ this reaction is essentially identical to the procedure of Kipping used in the 1920s as shown in Chart 2.41 An advanced modification of the reaction is to utilize a very small amount of crown ether or diglyme as an additive to activate sodium surface. The additives effectively improve polymerization yields, shorten polymerization times, and modify molecular weight distributions. ${ }^{35,42}$

Even for an optically active polymer prepared from an enantiopure monomer, it is difficult to identify whether the main chain in solution at a given temperature has an enantiopure helical conformation with either $P$ (plus, right-handed) or $M$ (minus, left-handed) screw sense, or is composed of a collection of diastereomerically and/or enantiomerically mixed $P$ - and $M$-helical motifs.

Optically active polysilanes, whose helical conformation is induced by chiral side chain interactions, are particularly suitable chiroptical polymers for elucidating the intrinsic nature of a helical polymer, since they embody a fluorophore and chromophore in the same main chain, showing intense UV, CD, and FL bands due to the silicon $\sigma \leftrightarrow$ silicon $\sigma^{*}$ transition around 300-400 nm. A combination of FL study with CD, UV, and/or NMR spectra of the main chain may be helpful in identifying screw sense, uniformity, rigidity, and proportion of $P$ - and $M$-screw sense of optically active polymers. The utility of the electronic spectroscopies lies in the fact that the photoexcited energy above the optical band gap relaxes to the lowest energy domain incorporated in a mobile main chain, from which emission then occur. ${ }^{30 d, 30 e}$ Moreover, samples of any desired molecular weight can be obtained by careful fractional precipitation, since the molecular weights of the polysilanes are widely distributed between $10^{3}$ and $10^{7}$. These unique characteristics permit a more straight- forward elucidation of the helix-property-functionality relationship. ${ }^{35}$

In the case of synthetic optically active polymers, the intuitive meaning of CD signal intensity is analogous to that of UV spectroscopy, with the additional dimension of the subtracted absorption between left- and right-circularly polarized light (L- and R-CPL). ${ }^{35,43}$ Absorption of light obeys the Beer-Lambert law and thus CD intensity is defined as $\Delta \varepsilon=\varepsilon_{\mathrm{L}}-\varepsilon_{\mathrm{R}}=\left(\mathrm{Abs}_{\mathrm{L}}\right.$ $\left.-\mathrm{Abs}_{\mathrm{R}}\right) / \mathrm{cl}$, where $\Delta \varepsilon$ is the molar circular dichroism intensity, $\varepsilon_{\mathrm{L}}$ and $\varepsilon_{\mathrm{R}}$ are the molar absorptivities for Land R-CPL, respectively, $\mathrm{Abs}_{\mathrm{L}}$ and $\mathrm{Abs}_{\mathrm{R}}$ are the absorbances of L- and R-CPL, $c$ is the molar concentration per repeat unit and $l$ is the path length. CD bands are commonly referred to as either positive or negative Cotton effects and the peaks as extrema. It is now useful to express a ratio of the CD intensity to that of the UV-visible absorption intensity. This is formalized in the dimensionless parameter, Kuhn's dissymmetry ratio, $g_{\text {abs }}=2 \Delta \varepsilon /\left(\varepsilon_{\mathrm{L}}+\varepsilon_{\mathrm{R}}\right)=\Delta \varepsilon / \varepsilon$, where $\varepsilon$ is the molar absorptivity per repeat unit. The dissymmetry ratio is a function of the magnetic dipole $(m)$ and electric dipole moments $(\mu)$ and the angle $\theta$ between them, such that $g_{\text {abs }}=4 \times R / D=4|m||\mu| \cos \theta\left(m^{2}+\mu^{2}\right)^{-1}$, where $R$ and $D$ are the rotational and dipole strengths, respectively. For chromophoric helical polymers, this $g_{a b s}$ ratio provides quantitative information on the helical characteristics such as screw pitch and sense, and diastereomeric and/or enantiomeric purities. Typical absolute magnitudes of $g_{\text {abs }}$ values for allowed transitions of chiral polymers range from $10^{-4}$ and $10^{-3}$. However, particular attention should be paid in the interpretation of $g_{\text {abs }}$ due to the possible presence of segments of opposite screw sense and helix reversals. This is because, if enantiomeric $P$ - and $M$-motifs absorb at the same wavelength, the magnitude of $g_{\text {abs }}$ would result in a smaller, or possibly zero value. In some case, apparent optical inactivity may arise from equal proportions of static and/or dynamic helical motifs under the measurement conditions.

Apparent split-type Cotton CD signals may arise from two possibilities. Firstly, in a polymer containing both diastereomeric $P$ - and $M$-screw senses with different absorption wavelengths, the positive and negative Cotton effects will be slightly offset with respect to each other. ${ }^{30 \mathrm{~d}}$ Secondly, for two adjacent chromophores, coupling between the electronic dipole transition moments will give bisignate $\mathrm{CD}$ signals which is the so-called exciton couplet signal. ${ }^{30 \mathrm{k}, 30 \mathrm{n}, 44}$ This couplet could be further classified as either an intramolecular interaction in the same molecule at a kink or upon chain folding, and/or an intermolecular interaction in aggregate phases. The sign of an exciton cou- 
plet signal affords a simple and convenient method for determining the chirality between the two interacting chromophores. ${ }^{30 \mathrm{k}, 30 \mathrm{n}, 44}$

\section{PREFERENTIAL SCREW SENSE HELICAL ORDERING OF POLYSILANES}

Although the main chain conformations of polysilanes may be classified as random coil, 73- (deviant) or $15_{7^{-}}$(transoid) helix, and all anti (achiral trans-planar zigzag) structures on the basis of recent works, ${ }^{45}$ it is now generally accepted that most polysilanes tend to adopt helical main chain structures, regardless of side groups, temperature, and solvents. However, most polysilanes do not show any Cotton effects in their CD spectra, due to the adoption of equal numbers of $P$ and $M$-segments, resulting in an average racemic helical conformation and optical inactivity. If, however, the helical main chain is induced by a chiral chemical influence to adopt a preferential screw sense with an enantiomeric excess of either $P$ - or $M$-screw sense, a Cotton effect will be seen in the CD spectrum. Thus the interaction between side groups, solvents, temperature, and helical main chain structures of polysilanes in solution may be probed by means of CD spectroscopy, combined with UV, FL, and ${ }^{29} \mathrm{Si} /{ }^{13} \mathrm{C}$ NMR spectroscopies and global properties measurements.

The helicity of polypeptides is inherent since the chiral stereogenic centers are incorporated into the main chain. ${ }^{29}$ The helical structures of poly(triphenylmethyl methacrylate $)^{7,16,29}$ and polyisocyanide ${ }^{8}$ produced by screw-sense selective polymerization are preserved through the stereochemical locking effect of bulky achiral side groups, while poly(trityl methacrylate) contains stereogenic centers in the main chain and has a highly isotactic structure. However, in the case of optically active polyisocyanates, polyacetylenes, polythiophenes, polyphenylenevinylenes, polycarbodiimides, and polysilanes, their helical structures are effectively induced by chiral side chains. In general, the latter group of optically active helical polymers can be obtained by (a) polymerization of achiral and/or prochiral monomers with enantiopure chiral catalyst or initiator, (b) chiral doping of optically inactive polymers with enantiopure chiral moieties, (c) separation of a racemic mixture of enantiomeric helices using chiral stationary phase chromatography for non-dynamic helical systems, (d) complexation of optically inactive polymers with non racemic chiral ligands, (e) postpolymerization functionalization with chiral moieties, (f) incorporation of enantiopure chiral end groups, (g) polymerization of non racemic chiral monomers, (h) copolymerization of non racemic chiral monomers with achiral monomers or with non racemic monomers, and (i) circularly polarized irradiation of polymers bearing racemic sensitizers. Synthetic techniques from (d) to (h) have been applied to prepare optically active polysilanes. ${ }^{30-34}$

\section{CORRELATION BETWEEN GLOBAL CONFORMATION AND OPTICAL CHARACTERISTICS}

The main chain mobility of $\pi$ - and $\sigma$-conjugating polymers is often connected to their electronic structures. Therefore, changes in the UV-visible absorption and chiroptical spectra are spectroscopically discernible as thermo-, solvato-, piezo-, or and electrochromisms. Although several polysilanes exhibit these remarkable phenomena, ${ }^{39}$ their structural origins were controversial until recently, due to the limited information available on the correlation between the conformational structures of the main chain, electronic state, and (chir)optical characteristics. In 1996, it was reported that in various polysilanes in $\mathrm{THF}$ at $30^{\circ} \mathrm{C}$, the main chain peak intensity per silicon repeat unit, $\varepsilon$ (Si repeat unit) ${ }^{-1} \mathrm{dm}^{3} \mathrm{~cm}^{-1}$, is closely connected to the viscosity index, $\alpha .^{46,47}$

Figure 1 shows the UV absorption spectra of four optically active poly(dialkylsilane)s featuring different chiral side groups in THF at $30^{\circ} \mathrm{C} .{ }^{30 \mathrm{k}, 35 \mathrm{a}} \mathrm{It}$ is evident that as the value of $\alpha$ increases from 0.59 to 1.25 in THF at $30^{\circ} \mathrm{C}$, the UV absorption intensity increases, whereas the full width at half maximum ( $f w h m)$ decreases. Thus, the degree of $\sigma$-conjugation, global conformation, and UV absorption characteristics in polysilanes are controllable by the appropriate choice of side groups.

Indeed poly[n-hexyl-( $S$ )-4-methylpentylsilane] $\mathbf{2}$ with $\alpha=0.75$ is nearly randomly coiled, while poly[n-hexyl-( $S$ )-2-methylbutylsilane] 4 with $\alpha=1.25$ adopts almost a rod-like conformation; poly $[n$-hexyl(S)-3-methylpentylsilane] 3 with $\alpha=0.92$ shows an intermediate behavior between 2 and 4 . Recently quantitative estimates of the persistence length $(q)$ have been given: $85 \mathrm{~nm}$ for 4 in isooctane at $20^{\circ} \mathrm{C}$, but $6.2 \mathrm{~nm}$ for 3 in isooctane at $25^{\circ} \mathrm{C} .{ }^{28 \mathrm{~m}}$ The high $q$ value of $\mathbf{4}$ is comparable to that in stiff polyisocyanate with $(R)$-2,6-dimethylheptyl groups $(q=76 \mathrm{~nm})$. Comparative $q$ values are reported for other polymers: poly $(n$-hexyl isocyanate) $(20-43 \mathrm{~nm})$, aromatic polyamides $(20-50 \mathrm{~nm})$, and the double helix DNA $(\sim 60 \mathrm{~nm}) .{ }^{12 \mathrm{~b}, 12 \mathrm{e}}$ It is noted that poly[methyl- $(S)-2-$ methylbutylsilane] $\mathbf{1}$ has a shrunken shape for the reason discussed in later.

Thus, it is established that global structures of opti- 

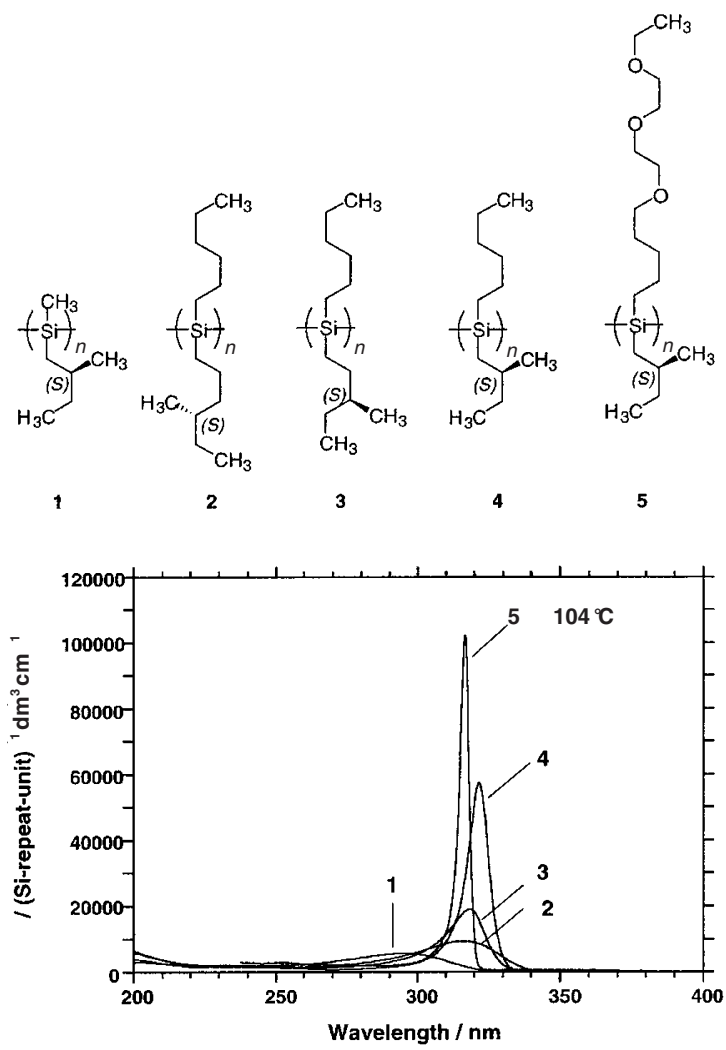

Figure 1. UV absorption spectra of four optically active polysilanes in THF at $30^{\circ} \mathrm{C}$ : Poly[methyl- $(S)-2$-methylbutylsilane] (1, $\alpha=0.59)$, poly $[n$-hexyl-( $S)$-4-methylpentylsilane $](2, \alpha=0.75)$, $\operatorname{poly}[n$-hexyl- $(S)$-3-methylpentylsilane $](3, \alpha=0.92)$, poly $[n$ hexyl-(S)-2-methylbutylsilane] $(\mathbf{4}, \alpha=1.25)$. For comparison, UV absorption spectrum of poly[6,9,12-trioxatetradecyl-( $S)$-2-methylbutylsilane] (5) in ethanol at $-104^{\circ} \mathrm{C}$ is also displayed.

cally active polysilanes are significantly influenced by the position of the branching methyl group ( $\beta$ - or $\gamma-)$ at the chiral center in the side chain, and also solution temperature. These results led to a semi-empirical relationship between the main chain absorption characteristics and the global conformation of various polysilanes in solution.

Figure 2 shows a correlation between the values of $\varepsilon$, fwhm, and $\alpha$ of various poly(dialkylsilane)s and poly(alkylarylsilane)s in THF at $30^{\circ} \mathrm{C} .{ }^{46}$ The polymers include seven optically active poly(dialkylsilane)s with four different types of chiral $\beta$-, $\gamma$-, or $\delta$ branched alkyl substituents, twelve optically inactive poly(dialkylsilane)s with six different types of linear, $\beta$-, $\gamma$-, or $\delta$-branched substituents, and two optically inactive poly(alkylphenylsilane)s. For the polysilanes exhibiting $\lambda_{\max }$ ranging from 290 to $352 \mathrm{~nm}$, it is evident that with increasing $\alpha, \varepsilon$ increases exponentially, but fwhm decreases exponentially. From this semiempirical $\varepsilon-\alpha$-fwhm relationship, either value of $\varepsilon$ or fwhm renders information on the degree of chain coiling in solution at a given condition. This may be very useful for discussing the global shape of a polysilane

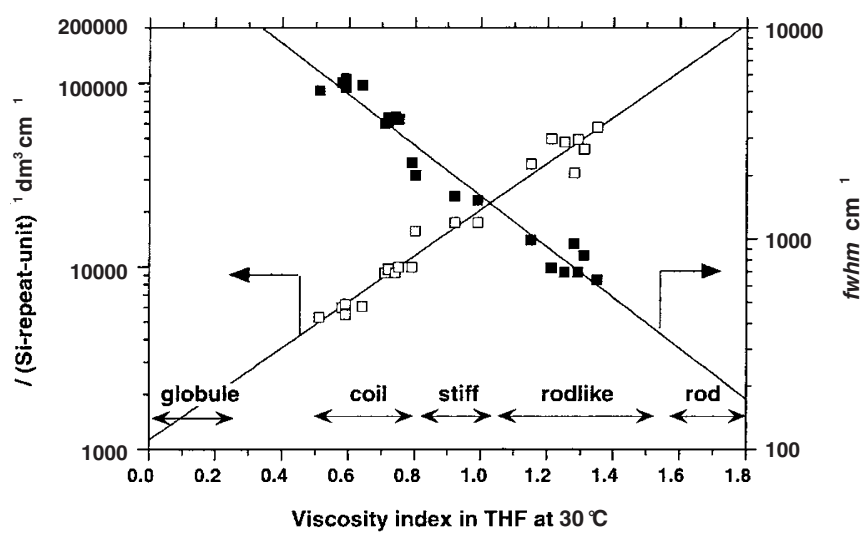

Figure 2. Correlation between UV peak intensity $(\varepsilon)$, full width at half maximum ( $f w h m$ ), and the viscosity index $(\alpha)$ of various poly(dialkylsilane)s and poly[alkyl(aryl)silane]s in THF at $30^{\circ} \mathrm{C}$.

in solution. We can obtain a value 150000 (Si repeat unit $)^{-1} \mathrm{dm}^{3} \mathrm{~cm}^{-1}$ for $\varepsilon$ of an ideal rod polysilane by extrapolating $\varepsilon$ using the above $\varepsilon$ - $\alpha$ relationship to the limiting $\alpha$ for an ideal rigid rod polymer of $1.7-1.8^{46,47}$ Indeed, as demonstrated in Figure 1, the $\varepsilon$ value for rodlike optically active poly[6,9,12-trioxatetradecyl- $(S)$-2methylpropyl)silane] (5) in ethanol progressively increases from 42000 with a fwhm of $800 \mathrm{~cm}^{-1}(8 \mathrm{~nm})$ at $323 \mathrm{~nm}$ to 102000 with a fwhm of $400 \mathrm{~cm}^{-1}(4 \mathrm{~nm})$ at $318 \mathrm{~nm}$ when the temperature is cooled from 25 to $-104{ }^{\circ} \mathrm{C} .{ }^{30 \mathrm{k}}$ This is the highest $\varepsilon$ value among all polysilanes should correspond to an almost perfect rod structure of 5 at such low temperatures.

\section{OPTICALLY ACTIVE DIALKYLPOLYSILANES}

\section{Preferential Screw Sense Homopolymers with Enan-} tiopure Chiral Side chains

The first CD spectrum and optical rotation of optically active poly(dialkylsilane) copolymer featuring di- $(S)$-2-methylbutylsilane and di- $n$-pentylsilane repeat units in the solid film and in solution were briefly reported by Matyjaszewski in 1992. ${ }^{30 a}$ This was followed by detailed reports on the synthesis and chiroptical properties of the copolymers in solution and as solid films by Möller, Matyjaszewski, and coworkers in 1994 and $1995 .^{30 b, 30 c}$ In 1994, it was clarified that the most fundamental chiroptical features and conformational properties in a series of optically active poly(dialkylsilane) homopolymers comprising alkyl$(S)$-2-methylbutylsilane repeat units in solution. ${ }^{30 \mathrm{~d}-30 \mathrm{f}}$

Poly[n-decyl-( $S$ )-2-methylbutylsilane] (6) among those polysilanes showed ideal UV, CD, and FL spectral characteristics of a rod-like helical chromophore and fluorophore due to the preferential single-screw sense structure induced by side group interactions in 


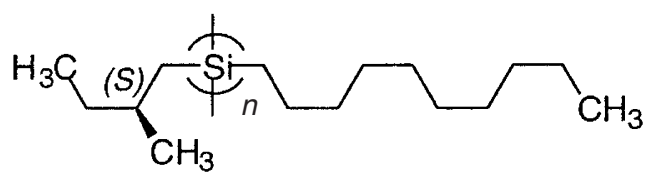

6
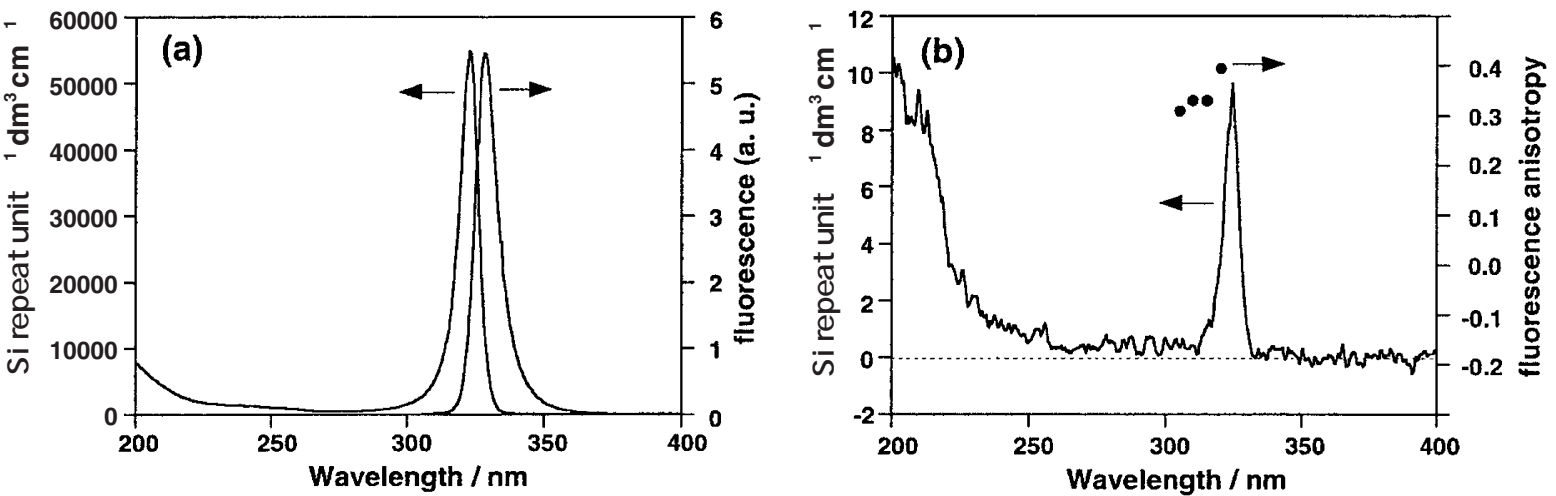

Figure 3. (a) UV and CD spectra and (b) FL spectrum and FL anisotropy (FLA) of poly $[n$-decyl-( $S)$-2-methylbutylsilane $]\left(6, M_{\mathrm{w}}=\right.$ $5.3 \times 10^{6}, M_{\mathrm{n}}=4.1 \times 10^{6}, \alpha=1.35$ in THF at $30^{\circ} \mathrm{C}$ ) in isooctane at $20^{\circ} \mathrm{C}$.
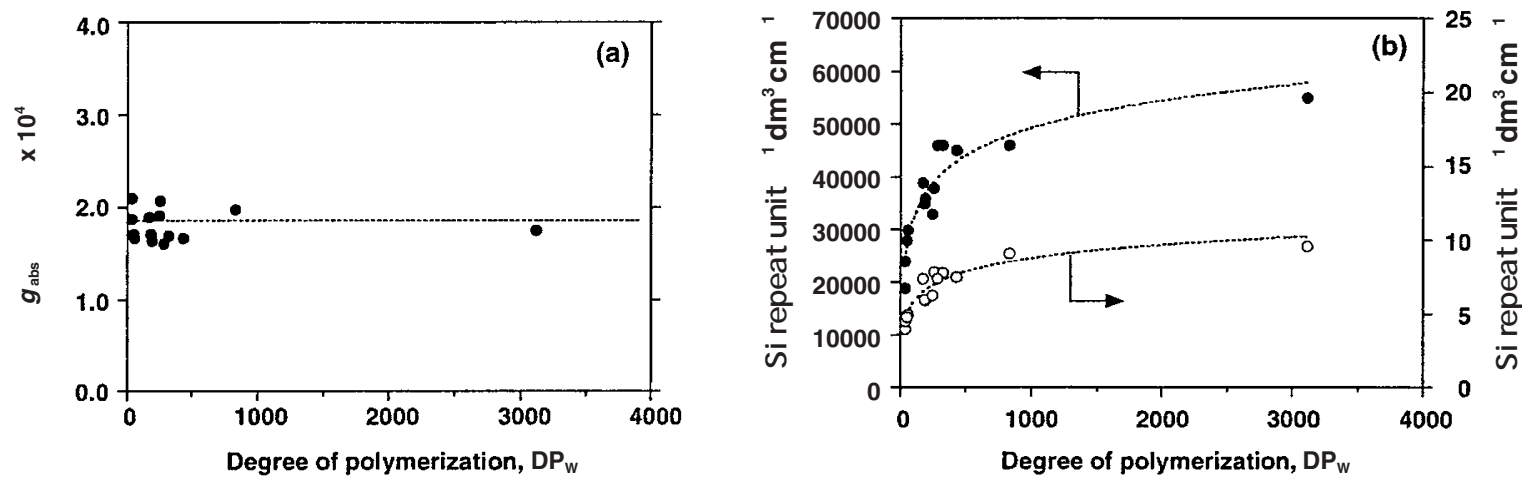

Figure 4. $\mathrm{UV}$ and $\mathrm{CD}$ bands characteristics at around $323 \mathrm{~nm}$ of fourteen samples isolated from nine poly $[n$-alkyl-( $S)-2-$ methylbutylsilane] derivatives as a function of the degree of polymerization $\left(\mathrm{DP}_{\mathrm{w}}\right)$ in isooctane at $20^{\circ} \mathrm{C}$ : (a) the dissymmetry ratio $\left(g_{\mathrm{abs}}\right)$ and (b) $\varepsilon$ and $\Delta \varepsilon$.

isooctane at $20^{\circ} \mathrm{C}$. As evident in Figure $3 \mathrm{a}$ and $3 \mathrm{~b}$, (i) 6 exhibits a very intense, narrow UV absorption with no discernible phonon side bands at $323 \mathrm{~nm}$, with $\varepsilon=55000$ (Si repeat unit) ${ }^{-1} \mathrm{dm}^{3} \mathrm{~cm}^{-1}$ and an $f w h m$ value of $8 \mathrm{~nm}(\varepsilon$ is about six times greater and the fwhm narrower by one sixth than typical random coil poly(dialkylsilane)s), (ii) the FL spectral profile at $328 \mathrm{~nm}$ is the mirror image of the UV and CD band profiles, (iii) the $\mathrm{CD}$ band profile fits fully within the UV spectrum, and (iv) the FL anisotropy (FLA) around the $323 \mathrm{~nm}$ UV-CD bands approaches the theoretical limit of 0.4 expected for the random distribution of a rod chromophore being collinear with the fluorophore in a rigid medium. It should be noted that these helical exciton transitions (due to a photoexcited electronhole pair) with negligibly weak electron-phonon coupling are unique, since these features are not seen in $\pi$ conjugating polymers or small molecules. These novel
UV-CD-FL-FLA characteristics of $\mathbf{6}$ could be the first demonstration of uniqueness among other optically active polysilanes and optically active organic polymers.

Furthermore, the UV, CD, FL, and FL excitation (FLE) spectral characteristics in isooctane at $20^{\circ} \mathrm{C}$ of poly[ $n$-alkyl-( $S)$-2-methylbutylsilane] derivatives were measured to elucidate the effects of the $n$-alkyl side group and main chain length. ${ }^{30 \mathrm{f}}$ It was concluded that the rod-like helical structure was retained, even when the $n$-alkyl side group length is increased from $n$-propyl to $n$-dodecyl and the main chain length is increased from 20 to 3000 silicon units. This was supported by the unique UV-CD-FL-FLE spectroscopic features of $\mathbf{6}$ and the fact that the $g_{\text {abs }}$ value of $\sim 1.9 \times 10^{-4}$ was almost independent of both $n$-alkyl side group length and silicon main chain repeat length, as is evident in Figure 4a. On the other hand, the peak intensities of UV and $\mathrm{CD}$ bands at $323 \mathrm{~nm}$ increase nonlinearly as the main 
chain repeat number increases and tend to reach constant values, as shown in Figure $4 \mathrm{~b}$, while the respective values of the UV peak wavelength and fwhm of the band converged to limiting values of $322 \mathrm{~nm}$ and $8 \mathrm{~nm}$ in isooctane at $20^{\circ} \mathrm{C}$.

It is noted that the dipole strength of UV absorption band is independent of the $n$-alkyl side group length and main chain repeat numbers. Actually, integration of the $323 \mathrm{~nm}$ UV absorption band of the poly[ $n$-alkyl- $(S)-2-$ methylbutylsilane] derivatives depends very weakly on the repeat numbers.

These results led to the important idea that, in the case of optically active polysilanes, the value of $g_{\text {abs }}$ ( $\left.=\int \Delta \varepsilon d \lambda / \int \varepsilon d \lambda\right)$ should be used to characterize helical structures such as the proportion of $P$ - and $M$-motifs and their regularity, rather than the values of $\Delta \varepsilon$ or optical rotation.

\section{AFM Single Molecular Imaging of Rod-Like Polysilane Structure}

Chain dimensional parameters of polymers such as the value of $q$ in dilute solution can be determined by well-established light scattering, sedimentation equilibrium, and viscometric techniques. ${ }^{12}$ Concerning solid state analysis, imaging of the detailed molecular structure of individual polymer chains on a solid surface is of growing interest in the design and control of the optoelectronic properties of functional polymers and nanomaterials. Recent advances in atomic force microscopy (AFM) and scanning tunneling microscopy (STM) have now enabled direct imaging of polymer molecules. For example, AFM images of a few insulating polymers such as cyclic DNA and random coiled polystyreneblock-poly(methyl methacrylate) on mica, poly(paraphenylene) on a gold surface, and twinned chiral polyacetylene on a highly oriented pyrolytic graphite substrate have been reported. ${ }^{48}$

In 1997, the first molecular AFM images of a high molecular weight sample of $6\left(M_{\mathrm{W}}=5.3 \times 10^{6}, M_{\mathrm{n}}=\right.$ $4.1 \times 10^{6}$; total molecular contour length $\sim 2000 \mathrm{~nm}$ ) on sapphire was reported by Ebihara et al. ${ }^{49 \mathrm{a}}$ The individual molecular image appears to essentially adopt rodlike structures accompanied by slight structural fluctuations. The rod-like objects consisted of several segments separated by kinks, in which the segment lengths are ranged from 150 and $800 \mathrm{~nm}$. These long segments may be responsible for the unique UV-CD-FL spectroscopic features of $\mathbf{6}$ and its derivatives. Although there may exist a certain gap in stereochemistry between polymers and small molecules, such AFM observation of polysilanes with different degree of stiffness may permit to narrow this gap. AFM/STM studies of various polymers might be the challenging issue in the forthcoming polymer science and nanomaterial science. ${ }^{48,49}$ Recently, Furukawa, Ebata, and coworkers successfully obtained a similar AFM molecular image of 6 , one end terminus of which had been chemically anchored on a silicon substrate, as well as of other flexible polysilanes bonded on solid substrates. ${ }^{49 \mathrm{~b}-49 \mathrm{e}}$

\section{Importance of Rod-Like Structures for Nanoscience and Nanotechnology}

It is expected that polysilanes with various stiffnesses can serve as polymer models of quantumwire semiconductors with a $0.2 \mathrm{~nm}$ silicon atomic size width and different degree of wire structure fluctuation in nanoscience and nanotechnology research areas. ${ }^{30 f, 36,37}$ The advanced one-dimensional exciton theory for a semiconducting polymer infinite chain with different degrees of disorder in the electronic state demonstrated that the exciton absorption significantly weakens and broadens as the degree of structural disorder increases. ${ }^{37 f}$ The disorders should involve structural deviations in $\mathrm{Si}-\mathrm{Si}$ bond length, $\mathrm{Si}-\mathrm{Si}-\mathrm{Si}$ bond angle, and $\mathrm{Si}-\mathrm{Si}-\mathrm{Si}-\mathrm{Si}$ dihedral angle. Such deviations are considered to determine the persistence length, mean free path, the effective $\sigma$-conjugation length, and degree of delocarization of hole and electron carriers in the main chain. ${ }^{301}$ As exemplified in Figures 2-4, individual rod-like polysilanes in solution indeed afford ultimately intense, narrow exciton absorption and emission, which nicely fit to the theoretical simulation for a one-dimensional exciton in idealized semiconducting polymers, while coiled flexible polysilanes with an inhomogeneous electronic state afford a weak, broad exciton absorption along with sharp emission.

Further developments of AFM/STM instruments and measurement techniques should permit the direct characterization of the helical pitch, screw sense, helix reversal, and superstructures of individual molecules of polysilanes and polymers in the near future.

Evidence for Diastereomeric Helices and Recombination for Single-Handed Helix

Amongst poly[ $n$-alkyl- $(S)$-2-methylbutylsilane] homopolymers, only 1 exhibited a very different $C D$ spectrum around the UV band at $300 \mathrm{~nm} .{ }^{30 \mathrm{~d}, 30 \mathrm{e}}$ An apparent bisignate $\mathrm{CD}$ band with positive and negative Cotton effects was observed, as shown in Figure 5a. From a deconvolutional analysis of the CD and UV spectra, both tight $P$ - and loose $M$-helical segments assumed to coexist in the same main chain in different screw pitch and opposite screw sense helical motifs, since the $\alpha$ value of 0.59 for 1 in THF at $30^{\circ} \mathrm{C}$ is typical of a coiled conformation. ${ }^{48}$ To test this idea, a screw-sense-selective "cut-and-paste" experiment was 

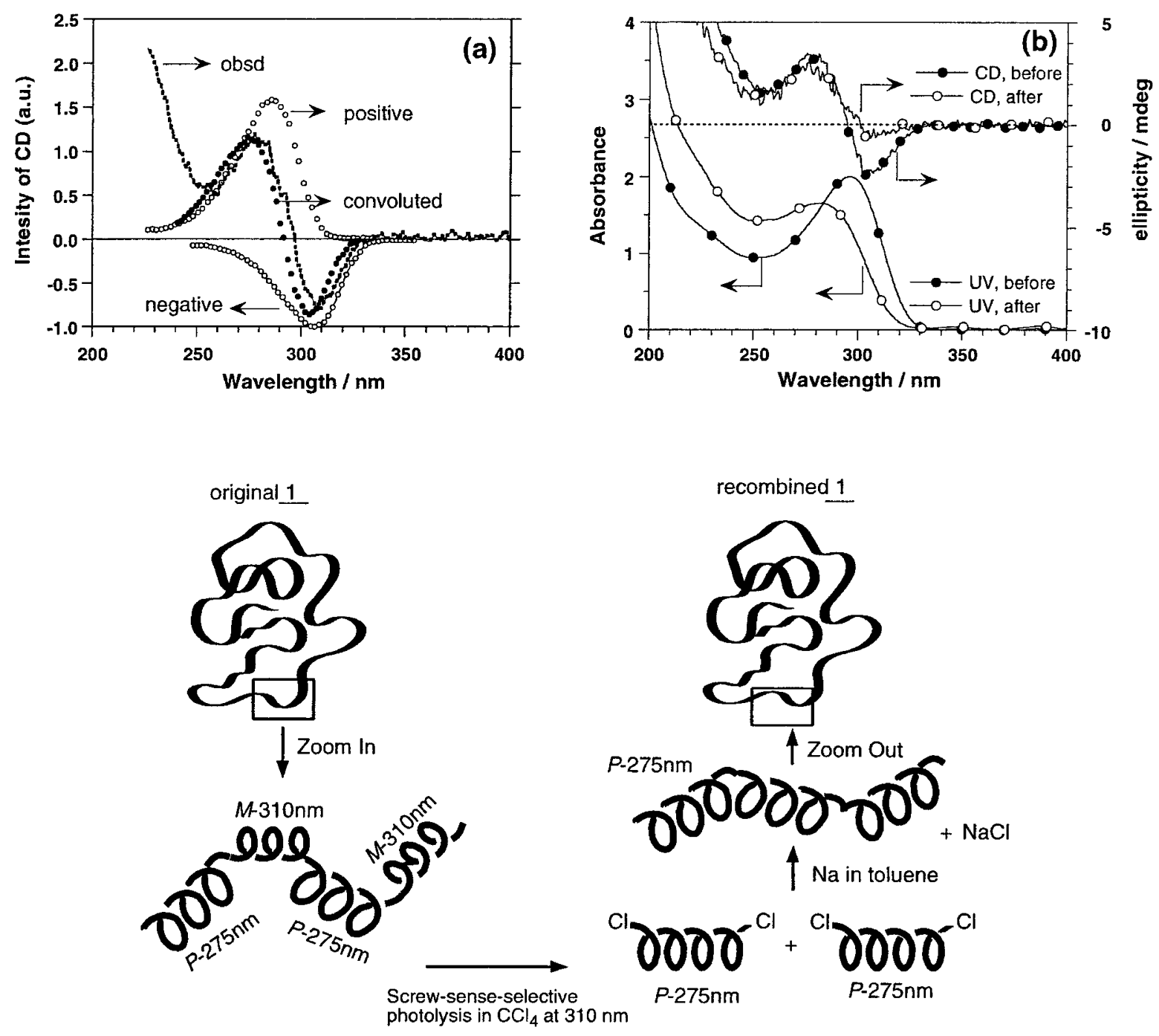

+ low-Mw species

(c)

Figure 5. (a) Original and deconvoluted CD spectra of original 1, (b) change in UV and CD spectra of original and recombined 1 (7) in isooctane at $25^{\circ} \mathrm{C}$ using the "cut-and-paste" technique. Key ideas for recombination of $\mathbf{1}$ with single screw sense from original 1 with diastereomeric helices with opposite screw-sense by the "cut-and-paste" technique, (c) reconstruction of $P$-screw-sense helical polymer 7 from 1 by a "cut-and-paste" procedure: (i) $M$-screw-sense photolysis of $\mathbf{1}$ in $\mathrm{CCl}_{4}$, (ii) formation of $P$-screw-sense telomers $\left(P_{\mathrm{w}} \sim 9\right)$ with $\mathrm{Si}-\mathrm{Cl}$ terminals, and (iii) Na-mediated re-condensation of the telomers. ${ }^{30 \mathrm{~d}, 30 \mathrm{e}}$

devised to prepare a modified 7 containing an almost single $P$-screw-sense. ${ }^{30 \mathrm{e}}$

As illustrated in Figure $5 \mathrm{c}$, this technique requires two steps: (i) $M$ - (or $P$-) screw-sense-selective photolysis at the longer $\lambda_{\max }$ region in $\mathrm{CCl}_{4}$ to form $P$ (or $M$-) screw-sense telomers with $\mathrm{Si}-\mathrm{Cl}$ termini, and (ii) Na-mediated re-condensation of the telomers in hot toluene. Indeed, formation of a modified 7 with an almost single screw sense was evidenced by significant changes in the CD and UV spectra after the "cut-andpaste" treatment, as shown in Figure $5 \mathrm{~b}$.

This knowledge and understanding may be helpful to characterize local conformations of other optically inactive polysilanes in solution. Indeed, all features of the UV and FL spectra, and FL anisotropy in $\mathbf{1}$ are very similar to those in most optically inactive, floppy polysilanes, such as poly(methyl- $n$-propylsilane) ( $\alpha=$ 0.62 in THF at $\left.30^{\circ} \mathrm{C}\right) .{ }^{30 \mathrm{e}}$ This latent chirality ${ }^{50}$ is assumed to result from the collective mixture of $P$ - and $M$-screw-sense coexisting in the same main chain of polymer, as a result of thermodynamic equilibrium, if $\mathbf{1}$ is thought to be a "snap shot" model for flexible polysilanes. ${ }^{30 \mathrm{e}}$

\section{Nonlinear Single-Screw-Sense Ordering in Copolymers} by the "Sergeants and Soldiers" Principle

The most outstanding feature concerning preferential screw-sense helical ordering in optically active copolymers is the "sergeants and soldiers" phenomenon named by Green et al. ${ }^{18 \mathrm{~b}}$ In this effect, a 


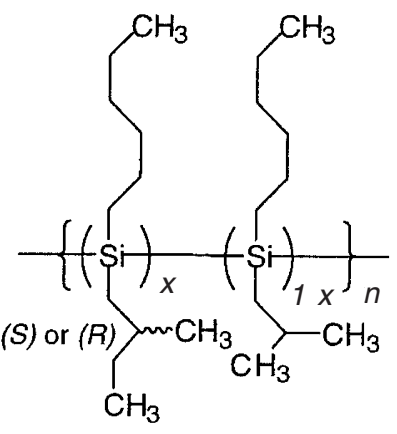

Copolymers 10 for $\mathrm{S}$ Copolymers 11 for $R$

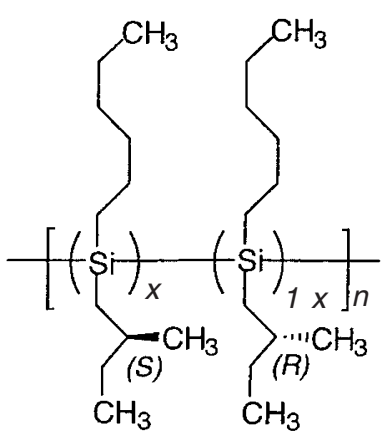

Copolymer 12

Chart 3. (left) Poly(dialkylsilane) copolymers (10 and 11) featuring enantiopure 2-methylbutyl and 2-methylpropyl side chains. (right) Poly(dialkylsilane) copolymers (12) featuring enantiomerically impure 2-methylbutyl side chains.

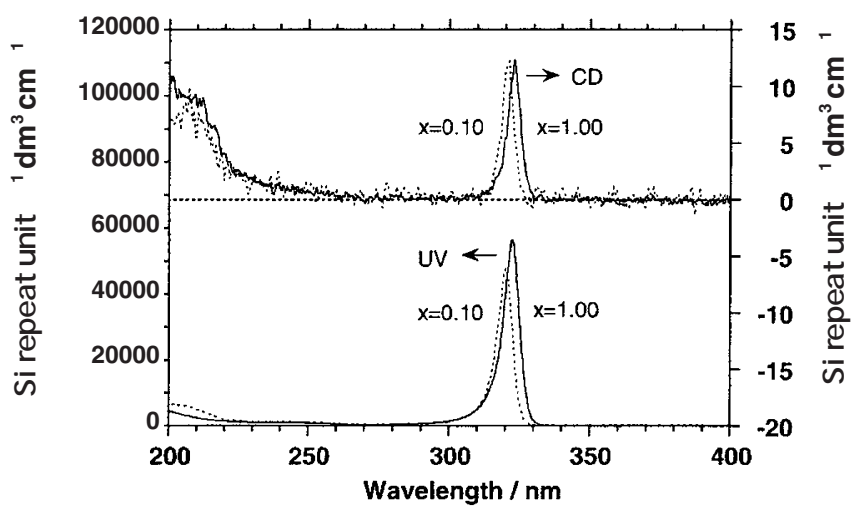

Figure 6. UV and CD spectra between homopolymer 4 (solid lines) and copolymer 10 (dotted lines) containing 10 mol\% of $(S)-2-$ methylbutyl groups in isooctane at $-5^{\circ} \mathrm{C}$.

minority of enantiopure chiral side groups cooperatively determines the screw sense of a helical main chain with the cooperation of a majority of achiral side groups. In other words, this phenomenon may be considered as "main chain chirality amplification" occurring in the polymer. Although this phenomenon was first reported by Pino et al.$^{9 \mathrm{a}, 9 \mathrm{~b}, 15 \mathrm{c}}$ in poly- $\alpha$-olefin copolymers, it has now also been established in copolymers of poly(alkylisocyanate)s, $9,18 \mathrm{~b}, 18 \mathrm{q}$ poly(arylisocyanate) ${ }^{18 \mathrm{n}}$ and polyacetylenes bearing chiral alkyl esters. ${ }^{19 \mathrm{k}}$

In the case of polysilane copolymers, although Möller, Matyjaszewski, and coworkers first reported that poly $\{($ bis $[(S)$-2-methylbutyl $]$ silane)-co-(di- $n$-pentylsilane $)\}$ (8) and poly\{(bis[(S)-2-methylbutyl]silane)-co-[(S)-2-methylbutyl- $n$-pentylsilane $]\} \quad$ (9) revealed no significant "sergeants and soldiers" phenomenon due to limited chiral side chain interactions in cyclohexane, ${ }^{30 \mathrm{~b}, 30 \mathrm{c}}$ which was evidenced by the fact that the CD signal intensity around $320 \mathrm{~nm}$ increased almost linearly with the mole fraction of the silicon repeat unit with $(S)$-2-methylbutyl group.

On the other hand, noticeable cooperative induc- tion of preferential screw sense was found in rod-like polysilane copolymers featuring $(S)$-2-methylbutyl and 2-methylpropyl groups (10) and (R)-2-methylbutyl and 2-methylpropyl groups (11) in isooctane (Chart 3). It is significant that these chiral and achiral groups are classified as $\beta$-branching alkyl groups and the resulting copolymers should adopt rod-like structures in solutions. Since the UV-CD spectral features of the copolymers are almost identical with those of $\mathbf{6}$ and the $\lambda_{\max }$ varies sensitively with changes in the mole fraction of chiral substituents and temperature, ${ }^{30 \mathrm{~g}, 35 \mathrm{a}, 35 \mathrm{~b}}$ the copolymer systems may be suitable for testing the "sergeants and soldiers" phenomenon by their CD-UV spectra.

Figure 6 compares the UV and CD spectra of 4 and 10 with $10 \mathrm{~mol} \%$ of $(S)$-2-methylbutyl groups in isooctane at $-5^{\circ} \mathrm{C}$. The UV-CD spectra of $\mathbf{1 0}$ are almost identical to those of $\mathbf{4}$, with the $\mathrm{CD}$ signal profile matching the corresponding UV absorption, except for their $\lambda_{\max }$ values. As expected, the UV and CD spectra of 11 with $(R)$-2-methylbutyl groups reveal almost identical features to those in 10, except for the sign of the CD band. 

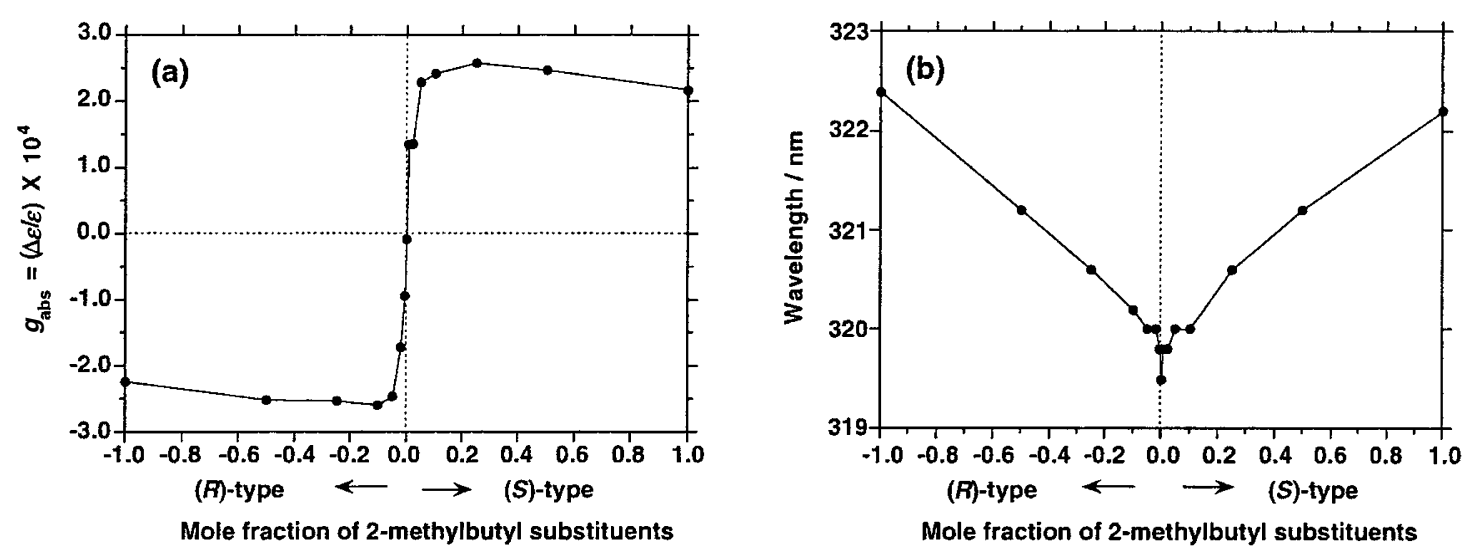

Figure 7. Values of (a) dissymmetry ratio $\left(g_{\mathrm{abs}}\right)$ and (b) $\lambda_{\max }$ of $\mathbf{1 0}$ and $\mathbf{1 1}$ as a function of mole fraction of $n$-hexyl- $(S)-2-$ methylbutylsilane and $n$-hexyl- $(R)$-2-methylbutylsilane in isooctane at $-5^{\circ} \mathrm{C}$.

The variation of the $g_{\text {abs }}$ values in copolymers $\mathbf{1 0}$ and 11 as a function of the mole fraction of $n$-hexyl- $(S)-2-$ methylbutylsilane or $n$-hexyl-( $R)$-2-methylbutylsilane is plotted in Figure 7a. It is obvious that only 510 mol\% chiral moieties in $\mathbf{1 0}$ and $\mathbf{1 1}$ almost definitively determines the screw sense. Moreover, even as little as $0.6 \mathrm{~mol} \%$ of chiral side groups induces a marked preferential screw sense helical conformation in the copolymers at temperatures below $0^{\circ} \mathrm{C}$. Presumably, the reason for no marked "sergeants and soldiers" effects in $\mathbf{8}$ and $\mathbf{9}$ is due to the greater flexibility of the di- $n$-pentylsilane moieties, since the $\alpha$ value of poly(di- $n$-pentylsilane) (13) may be similar to that of random coil poly(di-n-butylsilane) $(\mathbf{1 4}, \alpha \sim 0.71)$ and poly(di- $n$-hexylsilane) $(\mathbf{1 5}, \alpha \sim 0.74) .{ }^{46}$ Structural similarity between chiral and branched achiral side groups in copolymer systems seems to be vital in the promotion of effective "sergeants and soldiers" cooperativity.

The change in the $\lambda_{\max }$ values of $\mathbf{1 0}$ and $\mathbf{1 1}$ as a function of the mole fraction of $n$-hexyl- $(S)$-2methylbutylsilane or $n$-hexyl- $(R)$-2-methylbutylsilane is shown in Figure 7b. Although the value of $\lambda_{\max }$ redshifts progressively with increase in the fraction of the silane with chiral side chains, these features may be connected to a slight decrease in screw-pitch of the main chain. ${ }^{30 \mathrm{k}}$ Indeed, $a b$ initio calculations of parent polysilane $-\left(\mathrm{SiH}_{2}\right)_{n}$ - suggest that the main chain absorption wavelength (optical band gap) of polysilanes is progressively blueshifted with change in the main chain dihedral angle from $180^{\circ}$ (planar all-anti) to $60^{\circ}\left(4_{1}\right.$ helix), due to a decrease in $\sigma$-conjugation. ${ }^{51}$

Nonlinear Single-Screw-Sense Ordering by the "Majority Rule" Principle

Another significant cooperativity in preferential helical screw-sense optically active copolymers is the "majority rule" phenomenon. ${ }^{18 \mathrm{~h}, 18 \mathrm{q}}$ In this case, the screw sense of a helical main chain with enantiomer-

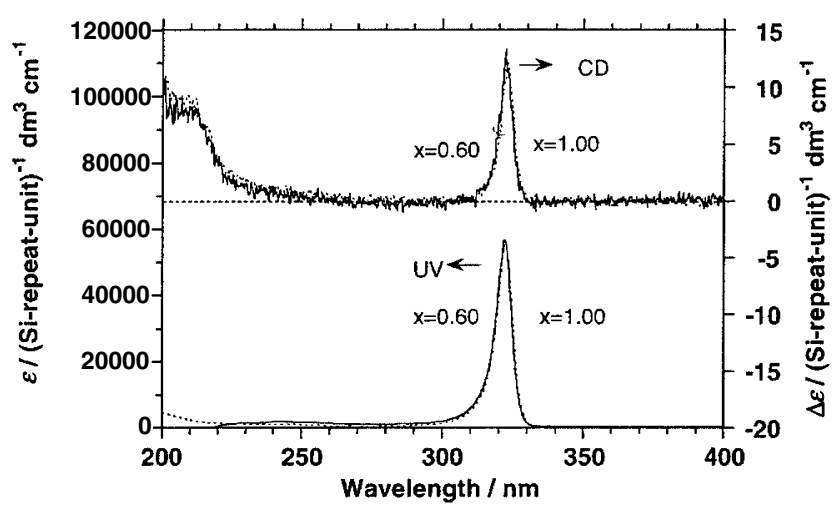

Figure 8. Comparison of UV and CD spectra between homopolymer 4 (solid lines) and copolymers $12(60 \mathrm{~mol} \%$ of $n$ hexyl- $(S)$-2-methyl-butylsilane and $40 \mathrm{~mol} \%$ of $n$-hexyl- $(R)-2-$ methylbutylsilane) (dotted lines) in isooctane at $-5^{\circ} \mathrm{C}$.

ically impure chiral side groups is controlled by the enantiomeric excess only. Since this phenomenon was first reported from poly- $\alpha$-olefins made of vinyl comonomers featuring enantio-impure chiral side groups by Pino et al, ${ }^{15 \mathrm{~b}}$ this "majority rule" has been already established in stiff polyisocyanates bearing enantioimpure chiral side chain., ${ }^{9}, 18 \mathrm{~h}, 18 \mathrm{q}$ This phenomenon is regarded as a certain "main chain chirality correction" characteristic of stiff polymer. Very similarly, noticeable "majority rule" effects were found in rod-like polysilane copolymer 12 containing $(S)$ - and $(R)$-2methylbutyl side groups in different proportions (Chart 3). ${ }^{28 g, 32 a, 32 b}$

A comparison of the UV and CD spectra between homopolymer 4 and copolymer $12(60 \mathrm{~mol} \%$ of $(S)$ - and $40 \mathrm{~mol} \%$ of $(R)$-2-methylbutyl side groups, $20 \%(S)$-ee of chiral side groups) in isooctane at $-5^{\circ} \mathrm{C}$ is shown in Figure 8. The UV and CD spectra of $\mathbf{1 2}$ are almost identical to those of 4 , the $\mathrm{CD}$ peak profile almost matching the corresponding UV absorption, including their $\lambda_{\max }$ values. As expected, the UV and CD spectra of the corresponding $(R)$-rich 12 copolymer containing $40 \mathrm{~mol} \%$ 

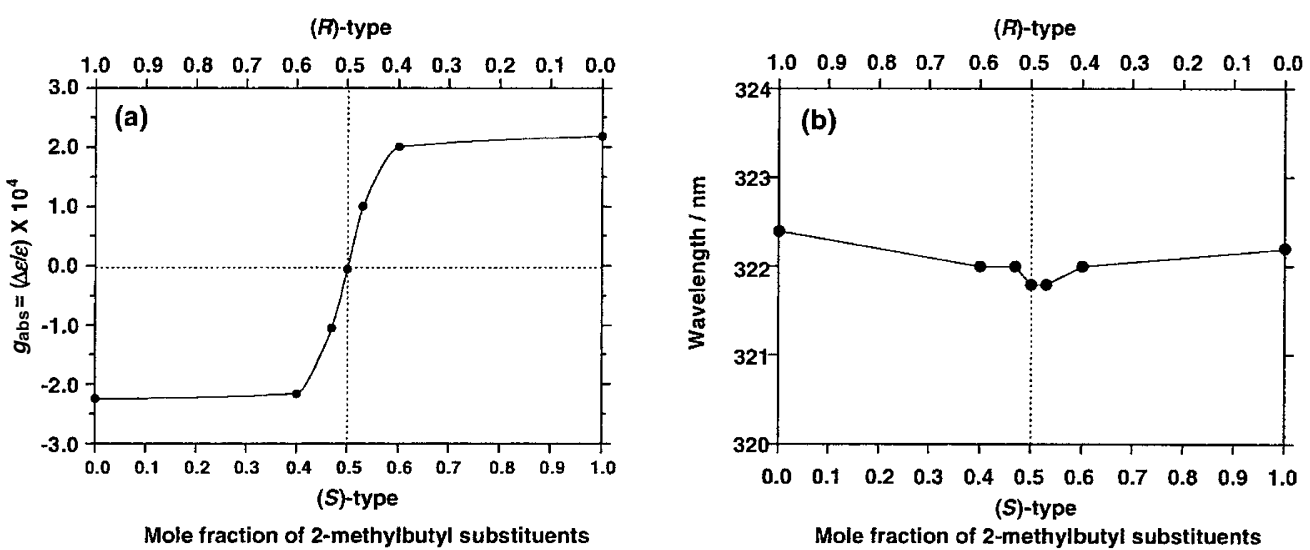

Figure 9. Values of (a) dissymmetry ratio and (b) $\lambda_{\max }$ in a series of copolymers $\mathbf{1 2}$ as a function of mole fraction of $n$-hexyl-( $\left.S\right)$-2methylbutylsilane and $n$-hexyl- $(R)$-2-methylbutylsilane in isooctane at $-5^{\circ} \mathrm{C}$.

of $(S)$ - and $60 \mathrm{~mol} \%$ of $(R)$-2-methylbutyl side groups $(20 \%(R)$-ee of chiral side groups) exhibit almost identical features to those in the $(S)$-rich 12 copolymer, except for the sign of the CD band.

As seen in Figure 9a, it is evident that only $20 \%$ ee of $(R)$ or $(S)$-2-methylbutyl group in $\mathbf{1 2}$ completely determines the overall screw sense and even only $6 \%$ $e e$ of $(R)$ or $(S)$-2-methylbutyl group induces a significant screw sense ordering in the copolymers. The structural identity between the $(S)$ - and mirror image $(R)$-chiral side groups and stiffer main chain in copolymer systems appears to be essential to induce an effective "majority rule" phenomenon. Also, as shown in Figure 9b, it is seen that, as the enantiopurity of $n$ hexyl-2-methylbutylsilane changes from $100 \%$ to $0 \%$ $e e$ in the copolymers, the value of $\lambda_{\max }$ is very weakly blueshifted, indicating minimal change in the screwpitch of the main chain, as suggested from the ab initio calculation. ${ }^{51}$

\section{Chiral Termini Effects by "Sergeants and Soldiers" Co- operativity}

The first single crystal X-ray structure, and CD and UV spectra of di- $n$-propylsilane decamer with enantiopure ( $R$ )-2-phenylpropyl (and $(S)$-2-phenylpropyl) termini (16) with preferential screw sense induced by cooperativity between achiral side groups and chiral end termini were reported by Kira and coworkers. ${ }^{30 \mathrm{~h}}$ This was followed by extension to high molecular weight poly(di- $n$-hexylsilane) with the same chiral end termini (17). ${ }^{30 \mathrm{j}}$ These works give important experimental evidence for the adoption of a 157 helical conformation in solution at lower temperature for $\mathbf{1 7}$, which was long believed to adopt an all-anti conformation of $\mathbf{1 5}$ in solution. ${ }^{38}$ Bisignate Cotton effects observed for $\mathbf{1 6}$ below $153 \mathrm{~K}$ and 17 below $233 \mathrm{~K}$ are considered to originate from an exciton couplet for two spatially interacting polysilane chromophores ${ }^{30 \mathrm{~h}, 30 \mathrm{j}, 44}$ which is com- posed of loose helical segments divided by one-handed approximately $90^{\circ}$ preferential screw sense kinks.

An intriguing end-termini effect in poly $(n$-hexyl2-methylpropylsilane) with ( $S$ )-2-methylbutyl termini (18) was found, though the value of $g_{a b s}$ is only about $-1 \times 10^{-5}$ which indicates a very weak induction power of preferential screw sense, ${ }^{30 \mathrm{~g}, 35 \mathrm{a}}$ it was apparent that the preferential screw sense of $\mathbf{1 8}$ was opposite to that of 4-6 bearing the same $(S)$-2-methylbutyl side groups. ${ }^{30 \mathrm{~d}, 30 \mathrm{f}, 30 \mathrm{k}}$

Another interesting chiral chain end effect may be the helical polymer block effect manifested in a synthetic block copolymer, poly(1,1-dimethyl-2,2-di$n$-hexylsilane)- $b$-poly(triphenylmethyl methacrylate) (19), reported by Sanji and Sakurai. ${ }^{30 s}$ The preferential screw-sense of the poly(di- $n$-propylsilane) block in 19 is induced by a $P$-helical poly(triphenylmethyl methacrylate) block in 19 in THF below $-20^{\circ} \mathrm{C}$, while the polysilane block at $20^{\circ} \mathrm{C}$ completely looses its optical activity. These results may be a new approach to design a thermally reversible, helix induction and chiroptical "on-off" switching function to the polysilane-based block copolymer through a helical programming protocol. Recently, it was first demonstrated that dissolving inherently optically inactive poly(methylphenylsilane) and poly(hexylmethylsilane) in optically active solvents, such as $(S)$-2-methyl-1-propoxybutane and $(S)$-(2methylbutoxymethyl)benzene, induces the polymer chains to adopt preferred helical screw senses through chiral solvation. ${ }^{32 \mathrm{~h}}$

\section{Cooperative Preferential Helix-Helix Inversion and Molecular Design}

A current challenging issue in the area of helical polymer and oligomer research is to design and realize dynamic chiroptical properties in response to external chemical and physical biases. ${ }^{8-10,12,28,34}$ The most 


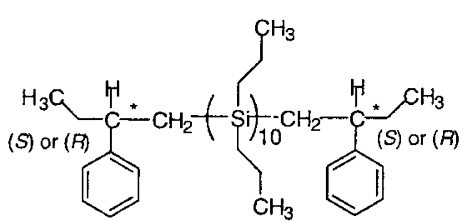

16

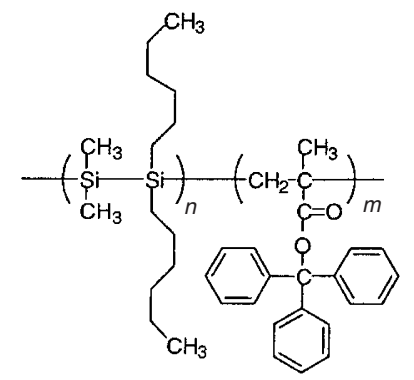

19
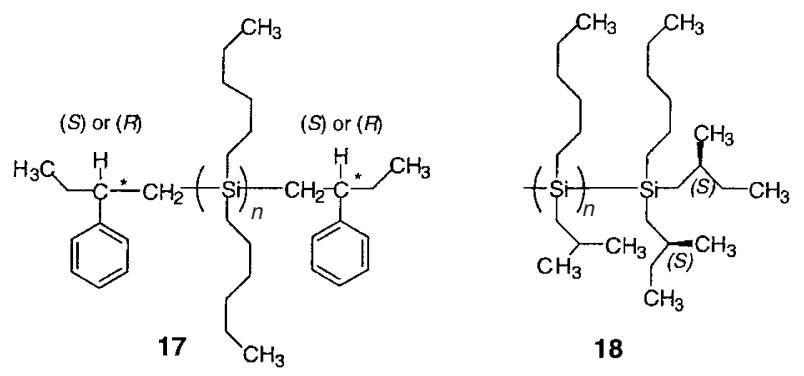

18

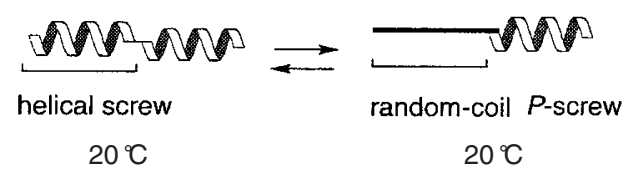

Chart 4. Poly(dialkylsilane) copolymers and oligomers (16-19) featuring chiral and helical end groups.<smiles>CC(C)CCC[C@H](C)CCC[Si](C)(C)CCCC(C)C</smiles><smiles>CC(C)CCC[C@H](C)CC[Si](C)(C)CC(C)C</smiles><smiles>CC(C)CCC[C@H](C)[C@H](C)CC[Si](C)(C)CC(C)C</smiles><smiles>CC(C)CCC[C@H](C)CCC[Si](C)(C)CCC[C@H](C)CCCC(C)C</smiles><smiles>CC[C@H](C)CCC[Si](C)(C)CCC[C@H](C)CCCC(C)C</smiles>

$20\left(T_{c}:-20^{\circ} \mathrm{C}\right)$<smiles>CCC(CC)C[Si](C)(C)CC[C@@H](C)CCCC(C)C</smiles><smiles>CC(C)CCC[C@H](C)CCCS(C)(C)CCC1CCCC1</smiles><smiles>CC[C@H](C)CC[Si](C)(C)CC[C@H](C)CCCC(C)C</smiles>

27<smiles>CCC(CC)CC[Sn](C)(C)CC[C@H](C)CCCC(C)C</smiles>

28<smiles>CC(C)CCC[C@H](C)CC[SiH2]CC1CCCC1</smiles>

29

Chart 5. Helix-helix transition (whose temperature is indicated in parenthesis) and non-helix-helix transition poly(dialkylsilane) homopolymers (20-29) featuring enantiopure $(S)$ - or $(R)$-3,7-dimethyloctyl group.

striking dynamic property for a helical polymer may be its chiroptical inversion associated with a helix-helix $(P M)$ transition in response to external physical and/or chemical stimuli. Although biochemists originally discovered this phenomenon almost three decades ago in synthetic DNA (driven by a change in $\mathrm{NaCl}$ concentration $)^{51}$ and poly $(l$-aspartic acid ester)s (biased by changes in temperature and organic acid), ${ }^{52}$ a few 

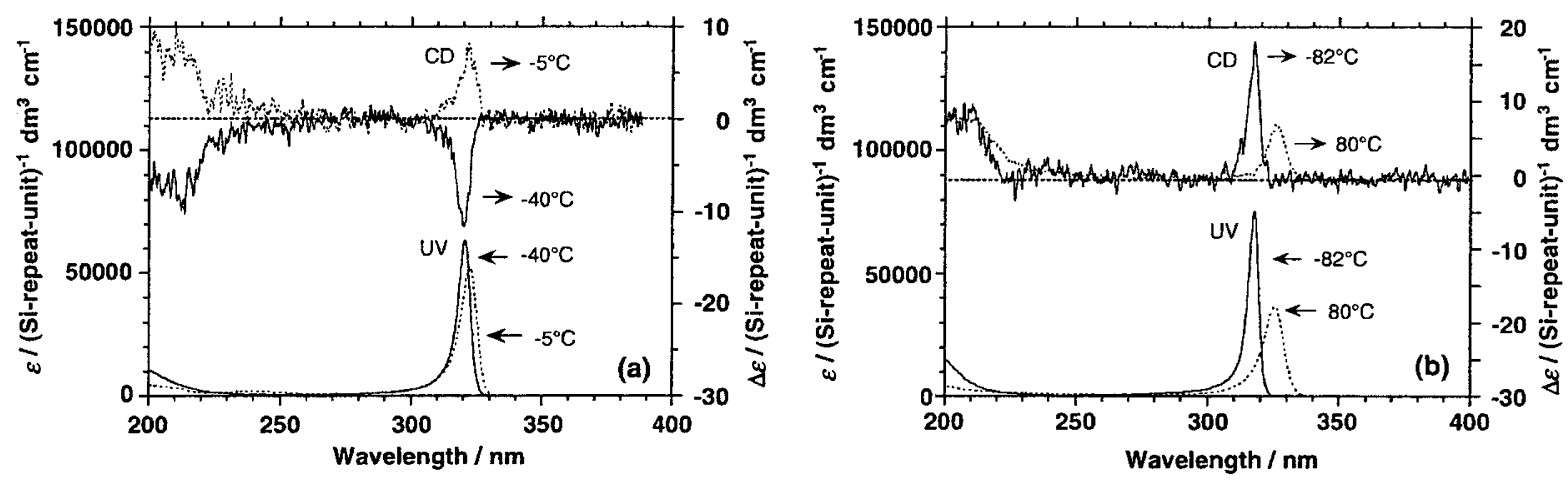

Figure 10. (a) $\mathrm{CD}$ and $\mathrm{UV}$ absorption spectra of poly[(S)-3,7-dimethyloctyl-3-methylbutylsilane $]\left(\mathbf{2 0}, M_{\mathrm{w}}=1.6 \times 10^{6}\right.$ and $M_{\mathrm{w}} / M_{\mathrm{n}}=1.53$ ) at $-40^{\circ} \mathrm{C}$ (solid line) and $-5^{\circ} \mathrm{C}$ (dotted line) in isooctane, (b) CD and UV absorption spectra of poly[ $(S)$ )-3,7-dimethyloctyl2-methylpropylsilane] $\left(\mathbf{2 1}, M_{\mathrm{w}}=4.2 \times 10^{4}\right.$ and $\left.M_{\mathrm{w}} / M_{\mathrm{n}}=1.51\right)$ at $-82^{\circ} \mathrm{C}$ (solid line) and $+80^{\circ} \mathrm{C}$ (dotted line) in isooctane.

synthetic organic polymers are reported to undergo a $P M$ transition in solution, or as solid films. For example, there are polyisocyanates, ${ }^{18 \mathrm{e}, 18 \mathrm{f}, 18 \mathrm{~m}, 18 \mathrm{p}, 18 \mathrm{t}}$

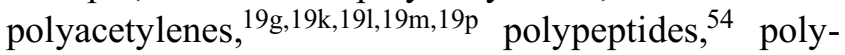
thiophenes, ${ }^{20 \mathrm{c}, 20 \mathrm{~d}, 20 \mathrm{j}}$ poly(triarylmethyl methacrylate), ${ }^{55}$ and calf thymus DNA. ${ }^{56}$ Recently, we found that certain poly(dialkylsilane) homopolymers and copolymers, ${ }^{31}$ poly(alkyl(alkoxylphenyl)silane) homopolymer, ${ }^{32 \mathrm{e}}$ and a poly(diarylsilane) copolymer $^{33 \mathrm{~b}, 33 \mathrm{c}}$ undergo a thermo-driven $P M$ transition in solution by controlling side chains and composition of copolymer.

Poly[ $(S)$-3,7-dimethyloctyl-3-methylbutylsilane] (20) may be classified in the family of rod-like helical homopolymers bearing enantiopure alkyl side groups, since 20 affords an intense, narrow UV absorption band, completely matching the corresponding $\mathrm{CD}$ and FL band mirror image profiles, similar to the rigid rod-like helical polysilane homo- and copolymers described above. However, 20 undergoes a thermodriven $P M$ transition in isooctane at $-20^{\circ} \mathrm{C}$, which is spectroscopically detectable as an inversion of the $\mathrm{CD}$ band.

Figure 10a and $10 \mathrm{~b}$ compare the $\mathrm{CD}$ and UV absorption spectra of 20 at -40 and $-5^{\circ} \mathrm{C}$ and of poly[ $(S)-3,7-$ dimethyloctyl-2-methylpropylsilane] (21) at -82 and $+80^{\circ} \mathrm{C}$ in isooctane. The positive-signed $\mathrm{CD}$ spectrum of 20 with $\lambda_{\text {ext }}$ of $320 \mathrm{~nm}$ at $-40^{\circ} \mathrm{C}$ is almost the inverse of the negative-signed CD spectrum with $\lambda_{\mathrm{ext}}$ of $322 \mathrm{~nm}$ at $-5^{\circ} \mathrm{C}$. It is evident that 20 undergoes a $P M$ transition between the two temperatures, though the helical motifs at -40 and $-5^{\circ} \mathrm{C}$ are energetically and spectroscopically non-equivalent. On the other hand, neither 21 nor its mirror-image poly[( $R)-3,7-$ dimethyloctyl-2methylpropylsilane] (22) undergo any such inversion of the CD spectra in isooctane between -90 and $+80^{\circ} \mathrm{C}$.

Polymers 6, 20, and $\mathbf{2 1}$ are thought to adopt rod-like 73 -helical conformations (dihedral angle of about $150^{\circ}$ or $210^{\circ}$ ) in solution, ${ }^{30 \mathrm{~d}, 30 \mathrm{~m}-30 \mathrm{w}}$ since these $\lambda_{\max }$ near $323 \mathrm{~nm}$ are almost identical to that of $\mathbf{1 3}$ and $\mathbf{1 4}$ adopt-

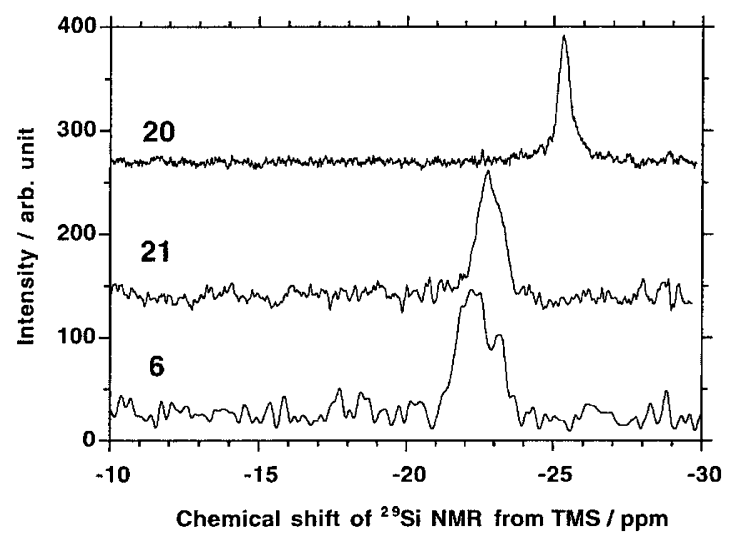

Figure 11. ${ }^{29} \mathrm{Si} \mathrm{NMR}$ spectra between (top) poly[(S)-3,7dimethyloctyl-3-methylbutylsilane] (20), (middle) poly[(S)-3,7dimethyloctyl-2-methylpropylsilane $]$ (21), and (bottom) poly $[n-$ decyl-(S)-2-methylbutylsilane] (6) in $\mathrm{CDCl}_{3}$ at $30^{\circ} \mathrm{C}$.

ing a 73 -helical form in the solid state. ${ }^{39 b}$ Indeed, it was concluded that 6 adopts $7_{3}$-helix in the solid state from $\mathrm{X}$-ray analysis. ${ }^{30 \mathrm{n}}$ Rod-like behavior of these polymers is expected by the high values of $\alpha$, the $\alpha$ values for 20 and 21 in $\mathrm{CHCl}_{3}$ at $30^{\circ} \mathrm{C}$ are 1.11 and 1.29 , respectively (for 6 in THF at $30^{\circ} \mathrm{C}$, the $\alpha=1.35$ ). ${ }^{31 \mathrm{a}}$ However, $\mathbf{2 0}$ is more flexible than $\mathbf{6}$ and $\mathbf{2 1}$, according to the following ${ }^{29} \mathrm{Si}$ NMR results.

Figure 11 displays the ${ }^{29} \mathrm{Si}$ NMR spectra of 20, 21, and 6 in $\mathrm{CDCl}_{3}$ at $30^{\circ} \mathrm{C}$. Since these polysilanes have two different types of side groups attached to the main chain, they may essentially comprise isotactic (it-), syndiotactic (st-), and heterotactic ( $h t-)$ sequences. Indeed, 6 exhibits a major signal at $-22.2 \mathrm{ppm}$ along with a weak signal at $-23.1 \mathrm{ppm}$, suggesting an almost single configurational sequence with a minor fraction of another sequence in the same main chain. Although $\mathbf{2 0}$ and $\mathbf{2 1}$ exhibit single ${ }^{29} \mathrm{Si}$ NMR signals near -23 to $-25 \mathrm{ppm}$, respectively, implying mainly it- or st- sequences, a remarkable difference in ${ }^{29} \mathrm{Si} \mathrm{NMR}$ linewidth $\left(\Delta v_{1 / 2}\right)$ between 20, 21, and $\mathbf{6}$ can be seen. The greater flexibility of $\mathbf{2 0}$ compared to $\mathbf{2 1}$ and $\mathbf{6}$ is 

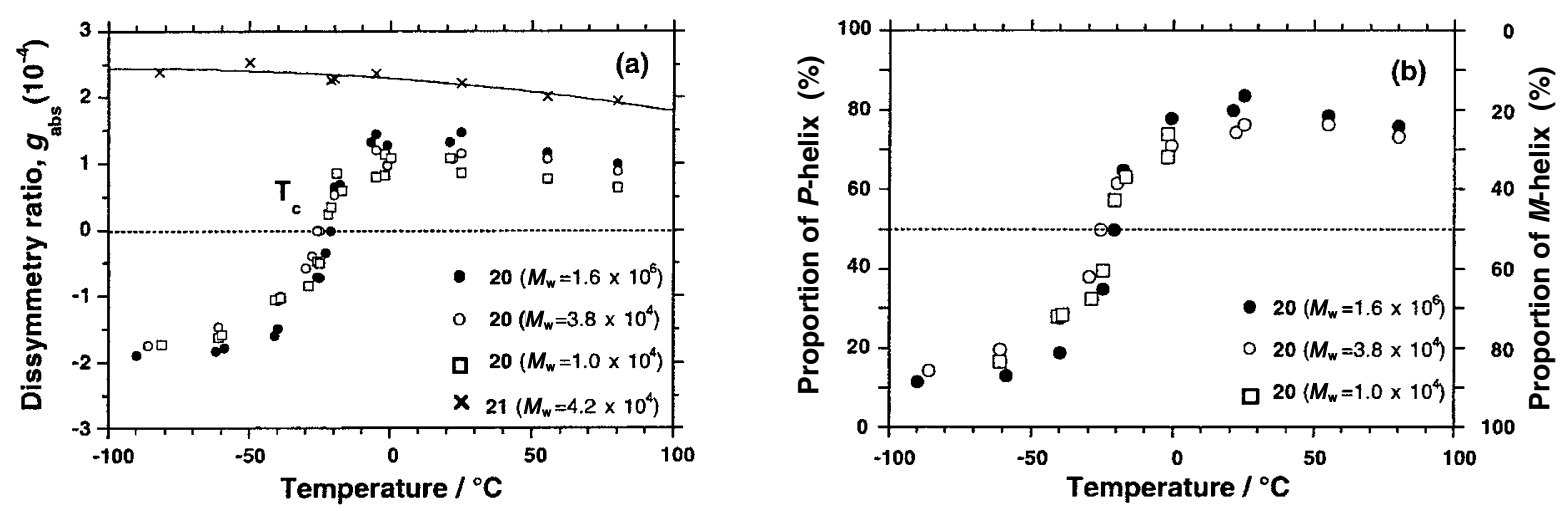

Figure 12. (a) Temperature dependence of the dissymmetry ratios of poly[(S)-3,7-dimethyloctyl-3-methylbutylsilane] (20) with three different $M_{\mathrm{w}}$ samples and poly[(S)-3,7-dimethyloctyl-2-methylpropylsilane] (21) which is assumed to be a purely $P$-helix in isooctane. The values of $M_{\mathrm{W}}$ were (i) $1.6 \times 10^{6}$ (closed circles), (ii) $3.8 \times 10^{4}$ (open circles), and (iii) $1.0 \times 10^{4}$ (open squares) for 20, and $4.2 \times 10^{4}$ (crosses and solid curve) for 21. (b) Temperature dependence of $P$ - and $M$-populations of $\mathbf{2 0}$ in isooctane by reference to the regression curve of $g_{\mathrm{abs}}$ values in $\mathbf{2 1}$.

suggested by the narrower $\Delta v_{1 / 2}$ : for $\mathbf{2 0}, \Delta v_{1 / 2}=29 \mathrm{~Hz}$ at $-25.3 \mathrm{ppm}$, while for $21, \Delta v_{1 / 2}=65 \mathrm{~Hz}$ at $-22.7 \mathrm{ppm}$ and for $\mathbf{6}, \Delta v_{1 / 2}=90 \mathrm{~Hz}$ at $-22.3 \mathrm{ppm}$.

Recently, we reported four more examples of $P M$ transition polysilanes in isooctane upon application of a thermal energy bias: poly $\{$ bis $[(S)-3,7-$ dimethyloctyl]silane $\}$ (23), ${ }^{31 \mathrm{~b}}$ poly $\{(R)-3,7$-dimethyloctyl-[(S)-3-methylpentyl]silane $\} \quad(\mathbf{2 4}),{ }^{31 \mathrm{c}, 31 \mathrm{~d}}$ poly$[(S)$-3,7-dimethyloctyl-(2-ethylbutyl)silane $] \quad(25),{ }^{31 \mathrm{e}}$ and poly[(S)-3,7-dimethyloctyl-(2-cyclopentylethyl)silane] $(\mathbf{2 6})^{31 \mathrm{ff}}$ have $T_{\mathrm{c}}$ of $2,-65,-7$, and $-33^{\circ} \mathrm{C}$, respectively. However, poly $\{(S)$-3,7-dimethyloctyl$[(S)$-3-methylpentyl $]$ silane $\}$ (27) $)^{31 \mathrm{c}}$ poly[ $(S)$-3,7-dimethyloctyl-(3-ethylpentyl)silane] (28), ${ }^{31 \mathrm{e}}$ and poly[(S)-3,7-dimethyloctyl-(1-cyclopentylmethyl)silane] (29) ${ }^{31 f}$ do not undergo a $P M$ transition. Recently, it was reported that the value of $q$ for $\mathbf{2 4}$ is as large as $103 \mathrm{~nm}$ in isooctane at $25^{\circ} \mathrm{C}$.

Although homopolymers bearing two $\gamma$-branched side groups per repeat unit may have a great possibility of undergoing a $P M$ transition, polymers with a combination of $\beta$ - and non-branched side groups or $\beta$ and $\gamma$-branched groups are unlikely to undergo a $P M$ transition. However, exceptionally 25 featuring $\beta$ - and $\gamma$-branched groups does undergo a $P M$ transition, ${ }^{31 \mathrm{e}}$ while 28 featuring two $\gamma$-branched groups does not. ${ }^{57}$ Additionally, although $\mathbf{2 3}$ and $\mathbf{2 7}$ have similar $\gamma$-chiral side groups, only $\mathbf{2 3}$ undergoes a $P M$ transition. In this case, the choice of handedness in the two $\gamma$-chiral side groups definitively determines the capability of a $P M$ transition. Thus, very minute modification and the choice of handedness in the side groups of polysilanes definitively determines the capability of the $P M$ transition and the value of $T_{\mathrm{c}}$. Beyond the factors discussed above, it is difficult to find general guidelines for designing polysilanes with a $P M$ transition capability.

\section{The Origin of Helix-Helix Transition}

Considering the variable temperature dependence of $\lambda_{\text {ext }}, \lambda_{\max }$, and intensities of $\Delta \varepsilon$ and $\varepsilon$ of $\mathbf{2 0}$ and $\mathbf{2 1}$ in isooctane, the $\lambda_{\text {ext }}$ and $\lambda_{\max }$ values of $\mathbf{2 0}$ and 21 linearly blue-shift from 325 to $318 \mathrm{~nm}$ as the temperature decreases from 80 to $-82{ }^{\circ} \mathrm{C}$. The $\varepsilon$ values of $\mathbf{2 0}$ and $\mathbf{2 1}$ increase monotonically from 40000 to 80000 over the same range. The former may be ascribed to a slight decrease in screw-pitch, deviating from an ideal $7_{3}$ helical structure, and the latter arises from a progressive increase in dimension of the main chain. However, the $\Delta \varepsilon$ value of $\mathbf{2 0}$ monotonically increases from ca. 3 at $+80^{\circ} \mathrm{C}$ to $c a .8$ at $-5^{\circ} \mathrm{C}$, goes to zero abruptly at $-20^{\circ} \mathrm{C}$, and then decreases to -15 monotonically at $-82^{\circ} \mathrm{C}$, while that of 21 monotonically increases from 7 to 18 . Mutual cancellation of positive and negative CD signals is indeed occurring at $T_{\mathrm{c}}$, resulting from an equal population of enantiomeric $P$ - and $M$-motifs with the same $\lambda_{\text {ext }}$ and $\lambda_{\max }$ values.

To quantitatively characterize the $P M$-populations using chiroptical characteristics, it is appropriate to use the $g_{\text {abs }}$ values of $\mathbf{2 0}$ at each temperature with reference to the regression curve of these $g_{\text {abs }}$ values in $\mathbf{2 1}$ which is assumed to adopt a purely $P-7_{3}$ helical structure, instead of the $\Delta \varepsilon$ value. The population analysis is based on the assumption that the weak temperature dependence of the $g_{\max }$ for $\mathbf{2 1}$ is due to a minute modification in the screw-pitch of the $P$-helix, rather than any formation of the $M$-helical motif.

Figure 12 plots the temperature dependence of the dissymmetry ratios and the $P M$-populations in three different $M_{\mathrm{w}}$ samples of $\mathbf{2 0}$ in isooctane based on the above analysis. It is evident that although the $P M$ populations in the three samples depend slightly on $M_{\mathrm{w}}$, a steep $P M$ transition clearly occurs at $-20^{\circ} \mathrm{C}$. The highest $M_{\mathrm{w}}$ polymer (closed circles) contains $12 \% P$ - 

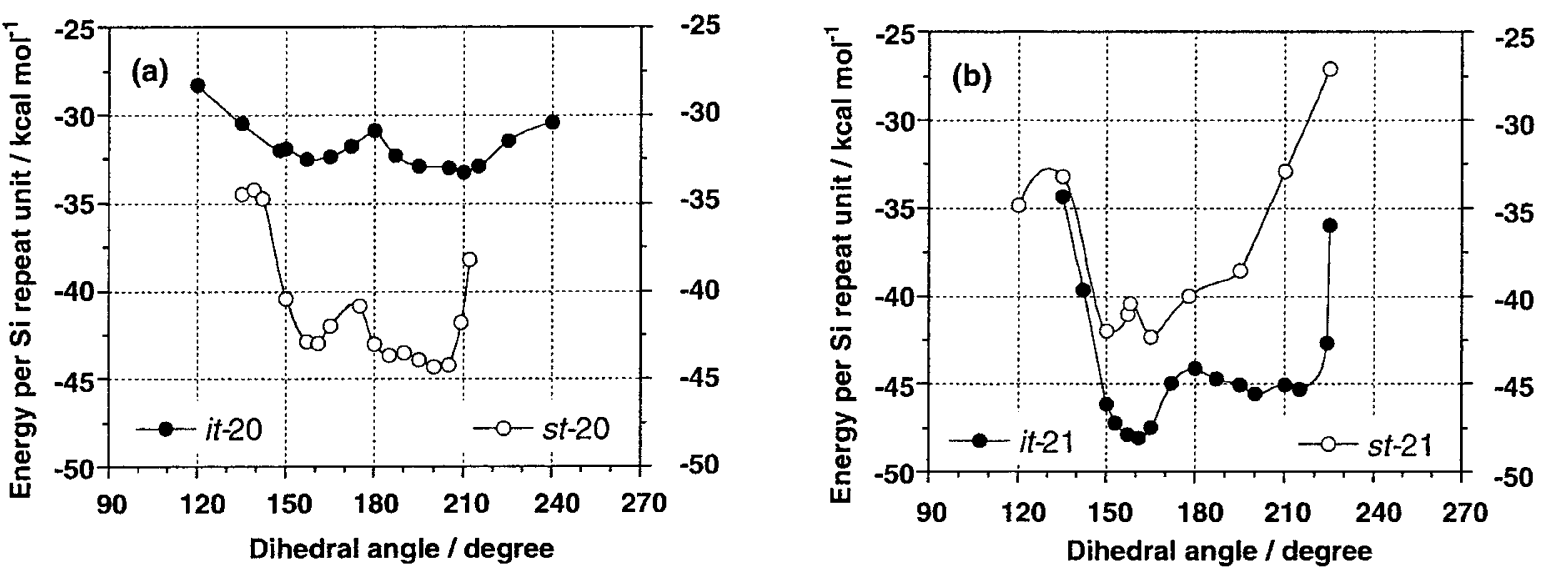

Figure 13. Molecular mechanics calculation. Main chain dihedral angle dependence on the potential energy (per repeat unit) of (a) it- and $s t$-(S)-3,7-dimethyloctyl-3-methylbutylsilane thirty-one repeat units with hydrogen termini (oligo-20), and (b) it- and st-(S)-3,7dimethyloctyl-2-methylpropylsilane thirty-one repeat units with hydrogen termini (oligo-21). Discover 3 (ver. 4.00, simple minimization, MSI) was used for this calculation.

and $88 \% M$-motifs at $-90^{\circ} \mathrm{C}$, while at $25^{\circ} \mathrm{C}$ these values invert to $84 \% P$ - and $16 \% M$-motifs. The medium and lower $M_{\mathrm{w}}$ samples contain $15 \% P$ - and $85 \% M$ motifs at $-90{ }^{\circ} \mathrm{C}$, while at $25^{\circ} \mathrm{C}$ these values are $76 \%$ $P$ - and $24 \% M$-motifs. However, the transition temperature width $\left(\Delta T_{\mathrm{c}}\right)$ tends to slightly broaden as $M_{\mathrm{w}}$ decreases. It is concluded that the $P M$ transition characteristics including $T_{\mathrm{c}}, \Delta T_{\mathrm{c}}$, and $P M$-populations weakly depend on the molecular weight, and the $P M$-transition characteristics may vary with segment length, for segments shorter than fifty silicon repeat units and/or the nature of two chain termini.

Although the origin of the $P M$ transition remains obscure, the marked differences in potential energy curves and main chain stiffness between $\mathbf{2 0}$ and $\mathbf{2 1}$ may be critical. Figure 13 shows the dihedral angle dependence on the potential energy of (S)-3,7-dimethyloctyl3-methylbutylsilane (thirty-one repeat units with hydrogen termini) (oligo-20) and (S)-3,7-dimethyloctyl(2-methylpropyl)silane (thirty-one repeat units with hydrogen termini) (oligo-21) for their it- and $s t$ sequences, respectively. An $i t$-oligo-20 clearly shows a double-well potential curve, i.e., two local energy minima with almost enantiomeric helices at dihedral angles of $P-157^{\circ}$ and $M-210^{\circ}$. The global minimum $M$ is slightly more stable than that of $P$ by about $0.67 \mathrm{kcal}$ per repeat unit and the barrier heights of the $M$ - and $P$-screw-senses are 2.3 and $1.7 \mathrm{kcal}$ per repeat unit, respectively. Also, st-oligo-20 has a similar doublewell potential curve at about $P-160^{\circ}$ and $M-200^{\circ}$ and the global minimum $M$ is slightly more stable than the corresponding $P$ by about $1.3 \mathrm{kcal}$ per repeat unit. The barrier heights for the respective $M$ - and $P$-screwsenses are about 4.6 and $3.4 \mathrm{kcal}$ per repeat unit. Thus, the calculation of oligo-20 suggests that both pseudo- enantiomeric $P$ - and $M$-motifs are likely to coexist in the same main chain at any temperature, regardless of microtacticity.

On the other hand, it-oligo-21 shows an unclear double well potential minima at dihedral angles of $P-160^{\circ}$ and $M-200^{\circ}$. The $P$-helix is much more stable compared to the corresponding $M$ by about $2.3 \mathrm{kcal}$ per repeat unit. The barrier heights of the respective $M$ and $P$ are about 3.9 and $1.4 \mathrm{kcal}$ per repeat unit, leading to the idea that $P$-helix might be more stable. A st-oligo-21 has an almost single well potential with dihedral angles of $P-160^{\circ}$. These calculations assume that the $P$-motifs of $\mathbf{2 0}$ and $\mathbf{2 1}$ are more stable at all temperatures, regardless of tacticity.

In order to discuss the origin of the $P M$ transition, some simple energy parameters of the $P$ - and $M$-states for oligo-20 and oligo-21 may be useful. Here, $\Delta G$ is the difference in free energy between the $P$ - and $M$ states, whereas $\Delta H$ and $\Delta S$ are the differences in enthalpy and entropy between the $P$ - and $M$-states, respectively.

$$
\Delta G=G_{\mathrm{P}}-G_{\mathrm{M}}=\Delta H-T \Delta S=H_{\mathrm{P}}-H_{\mathrm{M}}-T\left(S_{\mathrm{P}}-S_{\mathrm{M}}\right),
$$

where $\Delta H=H_{\mathrm{P}}-H_{\mathrm{M}}, \Delta S=S_{\mathrm{P}}-S_{\mathrm{M}}$

at $T_{\mathrm{c}}, \Delta G=0$, then $T_{\mathrm{c}}=\Delta H / \Delta S$

Using the values of $T_{\mathrm{c}}=253 \mathrm{~K}$ of 20 and $\Delta H=$ $-0.67 \mathrm{kcal}$ per repeat unit for oligo-20, one can obtain that $\Delta S=-2.6 \mathrm{cal} \mathrm{K}^{-1}$ at $T_{\mathrm{c}}$. Since the sign of the $\Delta G$ term below $T_{\mathrm{c}}$ is negative, $P$ is more stable than $M$, and vice versa. To design a thermo-driven $P M$ transition polysilane for switching at a desired temperature, it appears essential that the polysilane has a double-well potential curve, small $\Delta H$, and small potential barrier 
height. If the $\Delta S$ term contributes to the inversion capability of preferential screw-sense, $P$-polysilane in a low entropy state below $T_{\mathrm{c}}$ may take an ordered packing of side groups, whereas $M$-polysilane in the higher entropy state above $T_{\mathrm{c}}$ may have a disordered arrangement of side groups.

\section{Design to Helix-Helix Transition Characteristics by Copolymerization}

Thus, qualitative understanding of $P M$-transition characteristics and main chain stiffness of rod-like polysilanes led to the new idea that subtle structural modification of the achiral and chiral alkyl side groups controls critically the transition characteristics. Indeed, in a series of 20-based copolymers, poly $\{[(S)$ 3,7-dimethyloctyl-3-methylbutylsilane]-co-[(S)-3,7-dimethyloctyl-2-methylpropylsilane]\} (30) and poly$\{[(S)$-3,7-dimethyloctyl-3-methylbutyl-silane $]-c o-[(R)-$ 3,7-dimethyloctyl-2-methylpropylsilane]\} (31) (see Chart 6), $P M$-transition characteristics are controllable by the choice of side groups.

Figure 14 shows the temperature dependence of the $g_{\text {abs }}$ values and $P M$ populations in $\mathbf{3 0}(80 \mathrm{~mol} \%$ of $\mathbf{2 0}$ and $20 \mathrm{~mol} \%$ of $\mathbf{2 1}$ ), $\mathbf{2 0}$, and $\mathbf{3 1}(80 \mathrm{~mol} \%$ of $\mathbf{2 0}$ and
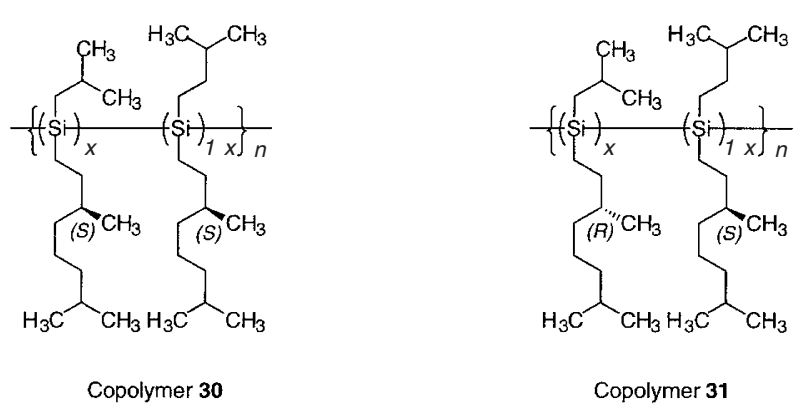

Chart 6. Two types of poly(dialkylsilane) copolymers (30 and 31) with helix-helix transition ability, featuring $(S)$ - and $(R)-3,7-$ dimethyloctyl, 2-methypropyl, and 3-methylbutyl side chains.
$20 \%$ of 22 ) in isooctane between -82 and $+80^{\circ} \mathrm{C}$. It is evident that, compared to the $T_{\mathrm{c}}$ of $\mathbf{2 0}$, that of $\mathbf{3 0}$ containing two identical $(S)$-chiral side groups decreases by $16^{\circ} \mathrm{C}$, whereas that of $\mathbf{3 1}$ containing the opposite $(S)$ - and $(R)$-chiral side groups increases by $16^{\circ} \mathrm{C}$. The $\mathrm{CD}$ spectral profile for the respective copolymers still matches the corresponding UV absorption spectrum at temperatures. However, as introduction of $\mathbf{2 1}$ or $\mathbf{2 2}$ to pure $\mathbf{2 0}$ causes an increase of the transition breadth $\Delta T_{\mathrm{c}}$ (defined as a difference between $T_{\mathrm{c}}$ up and $T_{\mathrm{c}}{ }^{\text {down }}$ ), due to the randomness of the two different silane repeat units incorporated in the same main chain.

The variation of the values of $T_{\mathrm{c}}$ and $\Delta T_{\mathrm{c}}$ is plotted in Figure 15, as a function of the mole fraction of 21 in 30, and 22 in 31 . As the mole fraction of 21 in 30, and 22 in 31 increases, the value of $T_{\mathrm{c}}$ changes nonlinearly from -64 to $+79^{\circ} \mathrm{C}$ and the value of $\Delta T_{\mathrm{c}}$ is nonlinearly broadened. As demonstrated in certain poly(alkylisocyanate) copolymer undergoing a $P M$ transition, ${ }^{18 \mathrm{t}}$ although a further mechanistic study

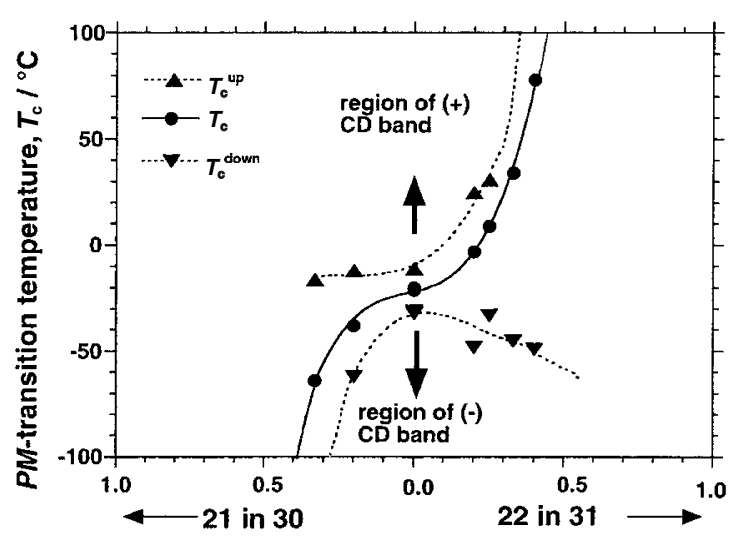

Mole fraction of 21 or 22 moiety in copolymers 30 and 31

Figure 15. Values of $T_{\mathrm{c}}$ and $\Delta T_{\mathrm{c}}$ as a function of mole fraction of $\mathbf{2 1}$ in 30, and $\mathbf{2 2}$ in $\mathbf{3 1}$ in isooctane. The $M_{\mathrm{w}}$ values in all the copolymers are ranged from $8.5 \times 10^{4}$ to $3.5 \times 10^{4}$.
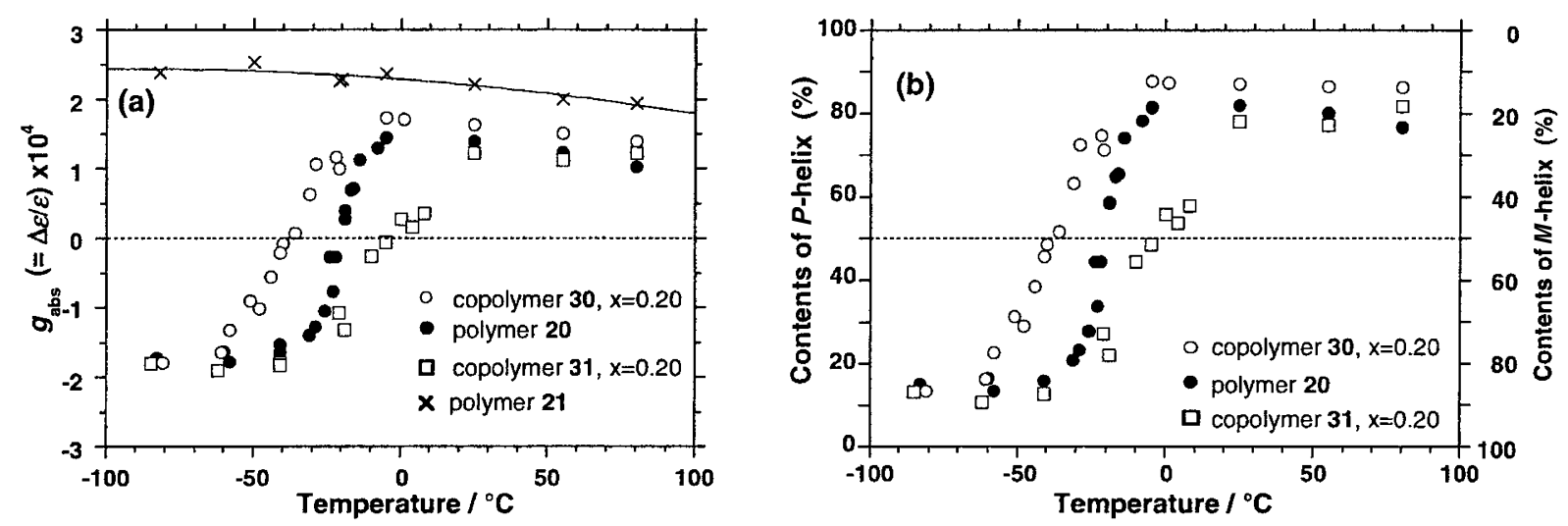

Figure 14. (a) Temperature dependence of the $g_{\text {abs }}$ values of $\mathbf{3 0}\left(80 \%\right.$ of $\mathbf{2 0}, 20 \%$ of $\mathbf{2 1}, M_{\mathrm{w}}=5.0 \times 10^{4}$, open circles $), \mathbf{2 0}\left(M_{\mathrm{w}}=\right.$ $3.8 \times 10^{4}$, filled circles), $\mathbf{3 1}$ ( $80 \%$ of $\mathbf{2 0}, 20 \%$ of $\mathbf{2 2}, M_{\mathrm{w}}=4.8 \times 10^{4}$, open squares), and $\mathbf{2 1}\left(M_{\mathrm{w}}=4.2 \times 10^{4}\right.$, crosses with a solid curve) in isooctane. (b) Temperature dependence of the $P M$-populations of the 30, 20, and $\mathbf{3 1}$ samples in isooctane by reference to the regression curve of $g_{\text {abs }}$ values in 21. 

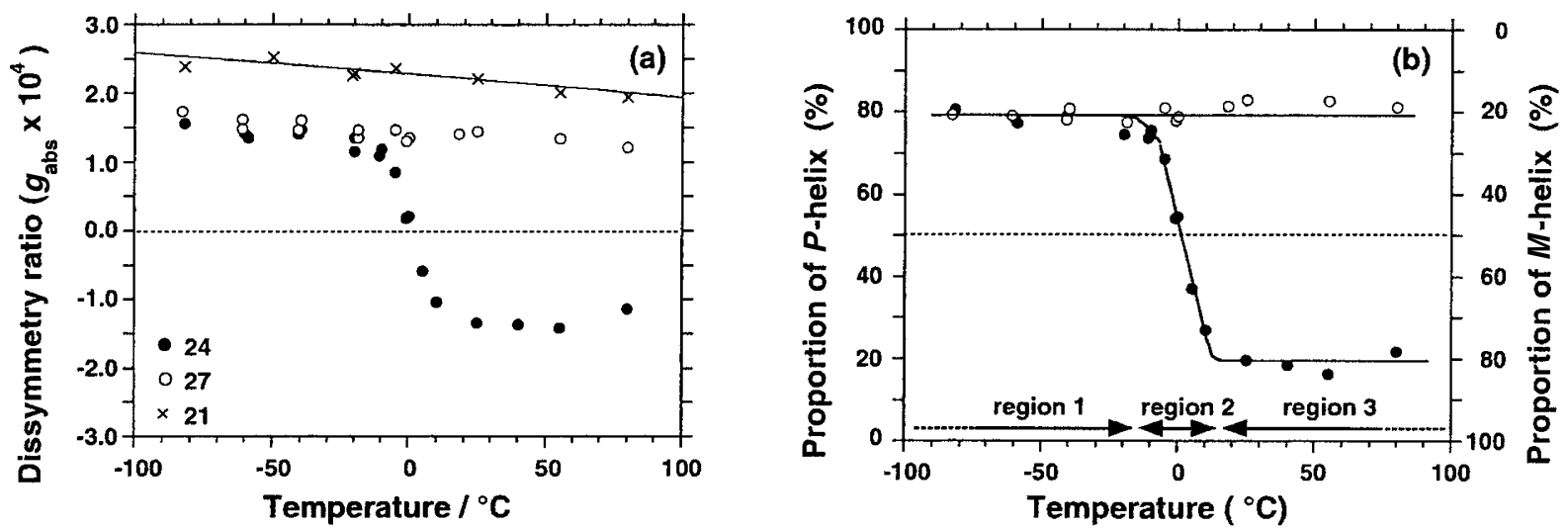

Figure 16. (a) Variable temperature $g_{\text {abs }}$ values of $\mathbf{2 4}\left(M_{\mathrm{w}}=5.8 \times 10^{6}, M_{\mathrm{n}}=3.4 \times 10^{6}\right.$, filled circles $), 27\left(M_{\mathrm{w}}=4.7 \times 10^{6}, M_{\mathrm{n}}=2.1 \times 10^{6}\right.$, open circles), and 21 (crosses) in isooctane in the range of -80 and $+80^{\circ} \mathrm{C}$. (b) Proportion of $P$ - and $M$-motifs of 24 (filled circles) and 27 (open circles) as a function of temperature.

is needed to clarify the realistic origin of the transition, presumably the two local free energy minimum potentials and entropy term in the free energy stability may be tunable by the choice of side chain chirality and the branch position in the side chain. Even in this case, the coexistence of $P$ - and $M$-motifs in the same main chain at any temperature may be essential for the capability of the $P M$-transition.

This knowledge and understanding may be helpful in designing $P M$ transition capability and characteristics in diverse screw-sense inversion related applications, such as a chiroptical switch and memory, a switchable chiral separation column, molecular recognition ability, and molecular motor in the future.

\section{Quantized and Superposed Helicities}

Designing and realizing miniature-scale computing devices are now the most challenging issues in nanoscience and nanotechnology. ${ }^{58}$ An optically active molecular switch and memory with bi- and multi-stable switching function associated with molecular motions such as twist, rotate, fold, shrink, stack, and shuttle may be a candidate as basic elements in these devices. ${ }^{59}$ Generally, great problems remain in the design and manufacture of such molecular elements, due to difficulties in the wiring of these elements with functions of "memory and storage", defined threshold, processing, and error correction. To date, understanding and measuring the superposition of quantum states in nanosystems based on semiconductors and superconductors directed towards the realization of quantum computing have been achieved theoretically and experimentally ${ }^{60}$ If the essence of "quantum computing" is the controlled mixing of two quantum states by physical and/or chemical biases, this may be also in the realm of molecular and polymer sciences. It may be noted that before these boom studies, several quantum chemists had al- ready discussed quantum tunneling and oscillation, and the preparation and detection of superposed optical activity for a hypothetical chiral molecule with a doublewell potential using an ultra short light pulse, although they did not extend their ideas to the possibility of chiral molecule-based quantum computing. ${ }^{61}$

As noted earlier, it is reasonable to use the dimension-less parameter to quantitatively evaluate the $P M$-population of $\mathbf{2 4}$ and $\mathbf{2 7}$ in solution by reference to the regression curve of the $g_{\text {abs }}$ values for $\mathbf{2 1}$, which is assumed to be in a purely $P$-motif over the temperature range, although the temperature dependence of the $g_{a b s}$ values in $\mathbf{2 1}$ shows a slight change of screw-pitch. Variable temperature $g_{\text {abs }}$ values and the proportion of $P$ and $M$-motifs of 24, 27, and 21 in isooctane between -80 and $80^{\circ} \mathrm{C}$ are plotted in Figure 16a and 16b. It is evident that $\mathbf{2 4}$ features three distinct switching regions, $1\left(-80\right.$ to $\left.-10^{\circ} \mathrm{C}\right), 2\left(-10\right.$ to $\left.+10^{\circ} \mathrm{C}\right)$, and $3(+10$ to $\left.+80^{\circ} \mathrm{C}\right)$. Polymer 24 in region 1 contains a constant $80 \% P$ and $20 \% M(60 \% P$ excess over $M)$, but contrarily in region 3 , it has $80 \% M$ and $20 \% P(60 \% M$ excess over $P$ ). However, 23 invariably contains $80 \%$ $P$ and $20 \% M(60 \% P$ excess $)$ over the entire temperature range. This behavior may be related to greater differences the potential energy curve between $\mathbf{2 4}$ and 27.

This led to the idea that the see-saw-like switching states between regions 1 and 3 may be quantized as the stationary state of $P$ - and $M$-superposition, but not due to $100 \%$ pure $P$ - and $100 \%$ pure $M$-states. These switching properties may be interpreted as the consequence of the superposition of $P$ - and $M$-helicities. Presumably, other $P M$-transition polysilanes, such as $\mathbf{2 0}$, 24, 25, and 26, should exhibit similar thermal energy biased superposed states between purely $P$ - and $M$ helicities in the main chain around helix-helix transition temperatures. ${ }^{31}$ 

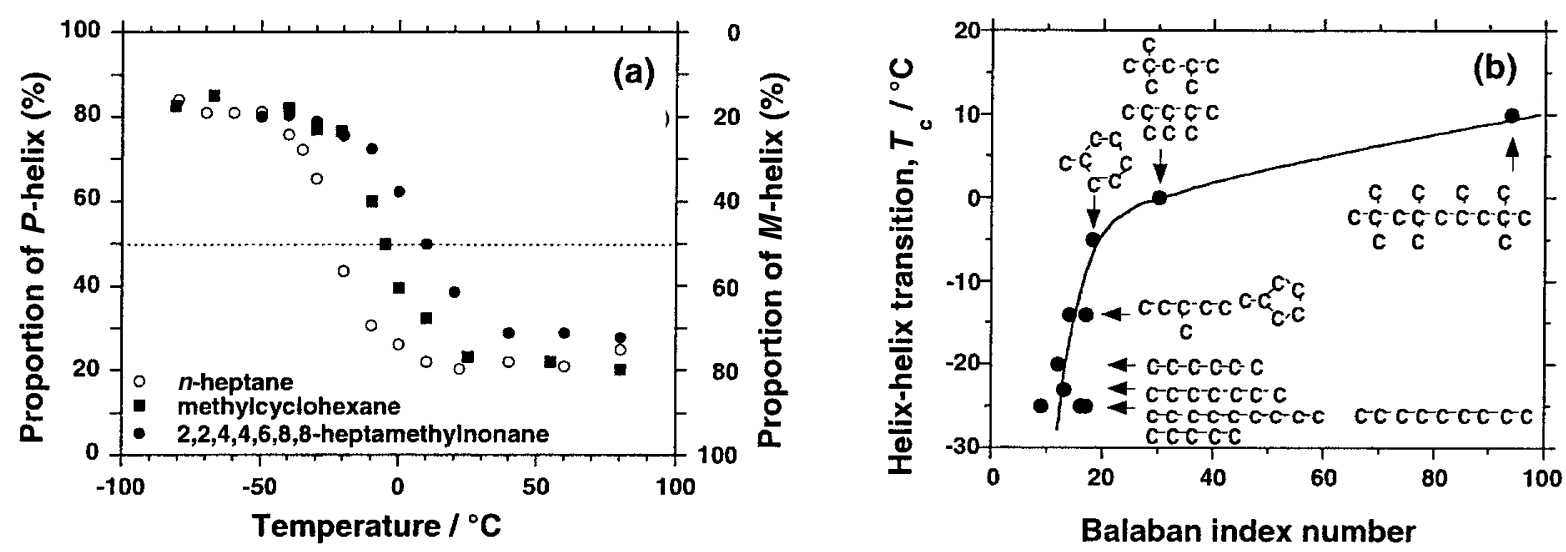

Figure 17. (a) Variable temperature $P$ - $M$ proportion of $\mathbf{2 4}\left(M_{\mathrm{w}}=7.4 \times 10^{4}, M_{\mathrm{n}}=2.5 \times 10^{4}\right)$ in 2,2,4,4,6,8,8-heptamethylnonane (filled circles), methylcyclohexane (filled squares), and $n$-heptane (open circles) in the range of -80 and $+80^{\circ} \mathrm{C}$. (b) Change in the $T_{\mathrm{c}}$ value of $\mathbf{2 4}$ $\left(M_{\mathrm{w}}=7.4 \times 10^{4}, M_{\mathrm{n}}=2.5 \times 10^{4}\right)$ as a function of degree of branching in a range of hydrocarbon solvent molecules in which the degree of branching is evaluated by the Balaban index number.

The most important feature of $\mathbf{2 4}$ exists in region 2 , because the superposed state almost linearly varies with thermal energy bias, ranging from $60 \% M$ - to $60 \% P$ excess. It is noted that $\mathbf{2 4}$ sensitively recognizes the topology of small molecules due to the two branched side chain structures. This superposed helical states linked by dynamic twisting motions may be considered to comprise a "dynamic memory" function, since, if the solvent molecules (external chemical bias) are taken away, the superposed state may modify the $P M$ population. This led to a change in $T_{\mathrm{c}}$ value with a range of non-polar hydrocarbon solvent molecules with different degrees of branching. This "solvent effect" of optically active polymers able to alter a preferential screw-sense was demonstrated in the optical activity of poly( $n$-hexyl isocyanate) induced by enantiopure chiral, racemic, and achiral solvents. ${ }^{18 \mathrm{k}}$

Figure 17 a compares the variable temperature $P-M$ population of $24\left(M_{\mathrm{w}}=7.4 \times 10^{4}, M_{\mathrm{n}}=2.5 \times 10^{4}\right)$ in three solvents with different degree of branching, including $n$-heptane (linear hydrocarbon), methylcyclohexane (cyclic hydrocarbon), and 2,2,4,4,6,8,8heptamethylnonane (highly branched hyfdrocarbon). The quantized helicity below and above $T_{\mathrm{c}}$, and the superposed helicity around $T_{\mathrm{c}}$ are conserved in these solvents. Although the value of $T_{\mathrm{c}}$ greatly varies with the degree of branching of the solvent, the $P-M$ population below $T_{\mathrm{c}}$ is insensitive to the degree of branching, but, above $T_{\mathrm{c}}$, however, it is sensitive to this.

Figure $17 \mathrm{~b}$ plots the effects of solvent topology on $T_{\mathrm{c}}$ for various solvents as a function of the Balaban index number which is a gauge of degree of branching. ${ }^{62}$ Quantitatively, although $T_{\mathrm{c}}$ increases nonlinearly with increasing the index number, straight chain alkyl molecules affords a lower $T_{\mathrm{c}}$, whereas branched chain alkyl molecules tend to give a higher $T_{\mathrm{c}}$ and branched cyclic solvents lie at intermediate values.

To explain these quantized and superposed helicities, cooperativity in coupled electronic and conformational transitions is assumed to be the origin of the step-like switching response to the thermal energy bias. In an electronically conjugating stiff helical polysilane with discrete energy levels in a double well potential, it is possible that the wavefunction of the lowest groundstate of an $M$-state electronically communicates with that of a $P$-state through quantum mechanical tunneling between the $P$ - and $M$-electronic states. The superposition of chirality and optical activity were discussed theoretically by Hund in 1927, 4a,63 and later by several quantum physicists for wavefunctions of hypothetical chiral molecules. ${ }^{61}$ It is necessary to invoke the presence of helix reversals in the case of helical polymers. The helix reversal, presumably consisting of several silicon-silicon single bonds (corresponding to a sub-nm barrier thickness), is the section in which the screw sense changes direction, and might act as a small tunneling barrier. ${ }^{4 \mathrm{a}, 31 \mathrm{c}}$ The reversal may play a crucial role in correcting errors from thermal noise through dynamic twisting motions from one screw sense to the other. The helix reversal may exist as a very short allanti, or transoid sequence. Apparent time-averaged optical inactivity at $T_{\mathrm{c}}$ should result from a rapid interconversion between $P$ - and $M$-motifs, which may be considered as oscillating helicity or dynamic pseudoracemization. In this quasi-stationary state, the wavefunctions of the superposed $P$ - and $M$-states $\left(P_{\mathrm{S}^{-}}\right.$and $M_{\mathrm{S}}$-states) may produce two splitting sub-levels, $\Psi=$ $1 / \sqrt{2\left[\Psi_{\mathrm{Ps}} \pm i \Psi_{\mathrm{Ms}}\right]}$, as illustrated in Figure 18b. This is a result of electronic coupling between the lowest energy levels of almost degenerate $P$ - and $M$-states in the same helical main chain.

Molecular mechanics calculations were also made on 

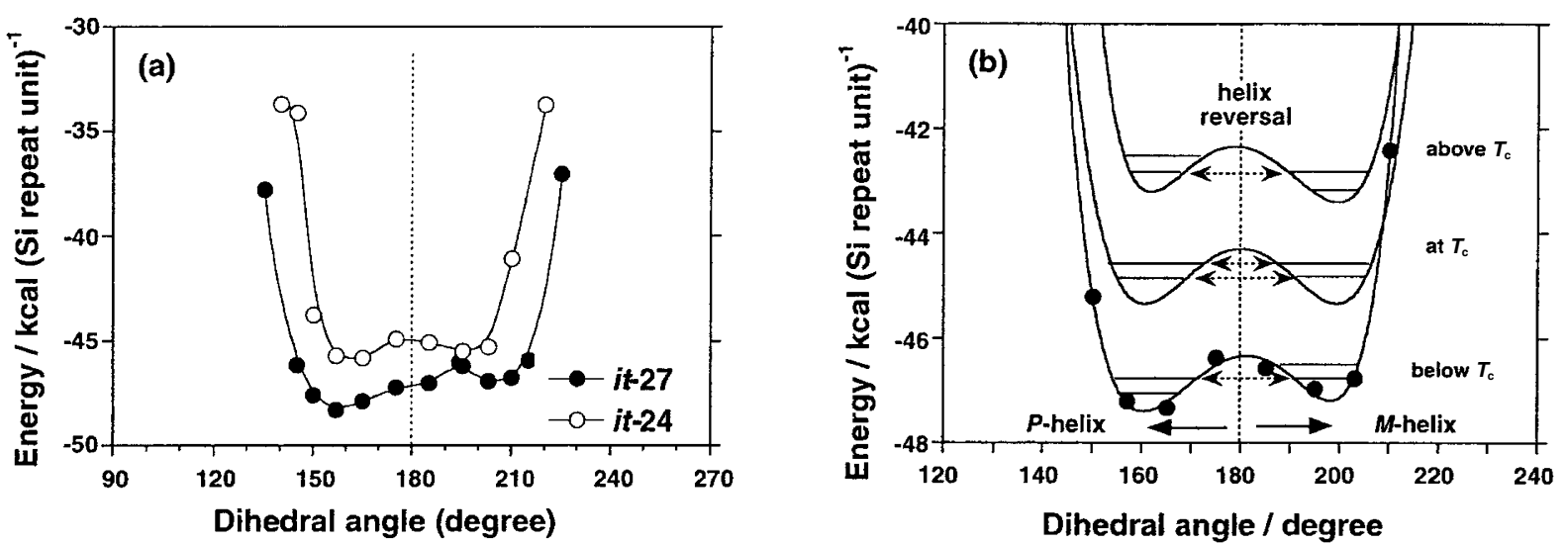

Figure 18. (a) Dihedral angle dependence of the potential energy for $i t-\mathbf{2 4}$ and $i t-\mathbf{2 7}$ model molecules with thirty-one repeating units and hydrogen termini (oligo-24 or oligo-27). (b) Potential energy as a function of main chain dihedral angles of it-oligo-24 with three switching regions. Below $T_{\mathrm{c}}$ (bottom trace with filled circles), at $T_{\mathrm{c}}$ (middle trace, hypothetical curve), and above $T_{\mathrm{c}}$ (upper trace, hypothetical curve). Dotted arrows indicate tunneling processes between wavefunctions of $P$ - and $M$-motifs existing in the same main chain. Discover 3, ver. 4.00 , simple minimization was used for this calculation.

31-mers of it-24 and it-27, with the results shown in Figure 18a. Both 31-mers are characterized by doublewell potentials. The $P$ minimum for $i t-\mathbf{2 4}$ is slightly deeper than the $M$ minimum, indicating that both $M$ and $P$-conformers may coexist. On the other hand, the $P$ minimum for $i t-27$ is significantly deeper than the $M$ minimum, suggesting that the chain conformation is predominantly $P$-helical. If the double-well potential of it-24 is assumed to change with temperature as shown in Figure 18b, the three temperature regions noted for $\mathbf{2 4}$ are qualitatively explained.

However recently it was found that the $P-M$ population $f_{\mathrm{P}}$ for $\mathbf{2 4}$ depends significantly on chain length as well as temperature. ${ }^{31 \mathrm{~d}}$ A similar molecular weight dependence was also found with 3. ${ }^{30 \mathrm{q}}$ These results have been well analyzed theoretically as illustrated in Appendix without recourse to the hypothetical potentials assumed in Figure 18b.

\section{Cholesteric Liquid Crystallinity of Helical Rod Polysi- lane}

Stiff rod-like helical polymers are expected to spontaneously form a thermotropic cholesteric liquid crystalline (TChLC) phase under specific conditions as well as a lyotropic liquid crystal phase. Recently, a certain rod-like poly(l-glutamate) with long alkyl side chains was reported to form a TChLC phase in addition to hexagonal columnar and/or smectic phases. ${ }^{64}$ These properties have already been observed in other organic polymers such as cellulose and aromatic polymers.

Recently, the persistence length, $q$, of $\mathbf{6}$ in isooctane was determined to be $70 \mathrm{~nm} .{ }^{30 \mathrm{r}}$ This value is similar to that of $\mathbf{4}(q=85 \mathrm{~nm})^{30 \mathrm{o}}$ and much longer than that of $3(q=6.2 \mathrm{~nm}),{ }^{30 \mathrm{o}}$ confirming the previous conclusion that polysilanes with $\beta$-branched alkyl side chains are much stiffer compared to those without such branching. The persistence length of helical polymers can be determined by the steepness of the internal rotation potential in the main chain. ${ }^{30 t}$ The slightly shorter $q$ of 6 than that of $\mathbf{4}$ implies that the longer nonbranched alkyl side chain makes the internal rotation double-well potential of the silicon main chain less steep.

Polymer $\mathbf{6}$ is the first TChLC polysilane in the range from 70 to $140^{\circ} \mathrm{C}$, as described below. The polymer 6 (of relatively low molecular weight: $M_{\mathrm{w}}=1.11 \times 10^{4}$, $D P_{\mathrm{w}}=46$, and $\left.M_{\mathrm{w}} / M_{\mathrm{n}}=1.19\right)$ was indeed shown to be a TChLC as proved by the iridescent colors observed due to the reflection of light from many helicoidal periods in randomly aligned textures. This is further supported by the observation using an optical microscope, of a Grandjean monodomain optical image including partly oily streaks between 70 and $140^{\circ} \mathrm{C}$, which are characteristic of the TChLC phase. ${ }^{30 n, 30 u}$ The TChLC phase of $\mathbf{6}$ was indeed proved by iridescent colors due to reflection light from many helicoidal periods in randomly aligned textures. This is further supported as the Grandjean monodomain optical image including partly oily streaks between 70 and $140^{\circ} \mathrm{C}$, which are characteristic of TChLC, by optical microscope observation.

Further evidence was obtained by directly observing the variable temperature $\mathrm{CD}$ reflection band characteristic of a cholesteric Grandjean domain, as shown in Figure 19a. The CD reflection spectra are fairly sharp at any temperature, and the reflection wavelength, corresponding to the optical pitch of the TChLC phase, greatly depends on sample temperature. The optical cholesteric pitch decreases progressively with temperature from $500 \mathrm{~nm}$ at $70^{\circ} \mathrm{C}$ to $1000 \mathrm{~nm}$ at $140{ }^{\circ} \mathrm{C}$. The positive sign of the $\mathrm{CD}$ reflection band indicates an $M$ helicity of the cholesteric phase. The optical pitch be- 

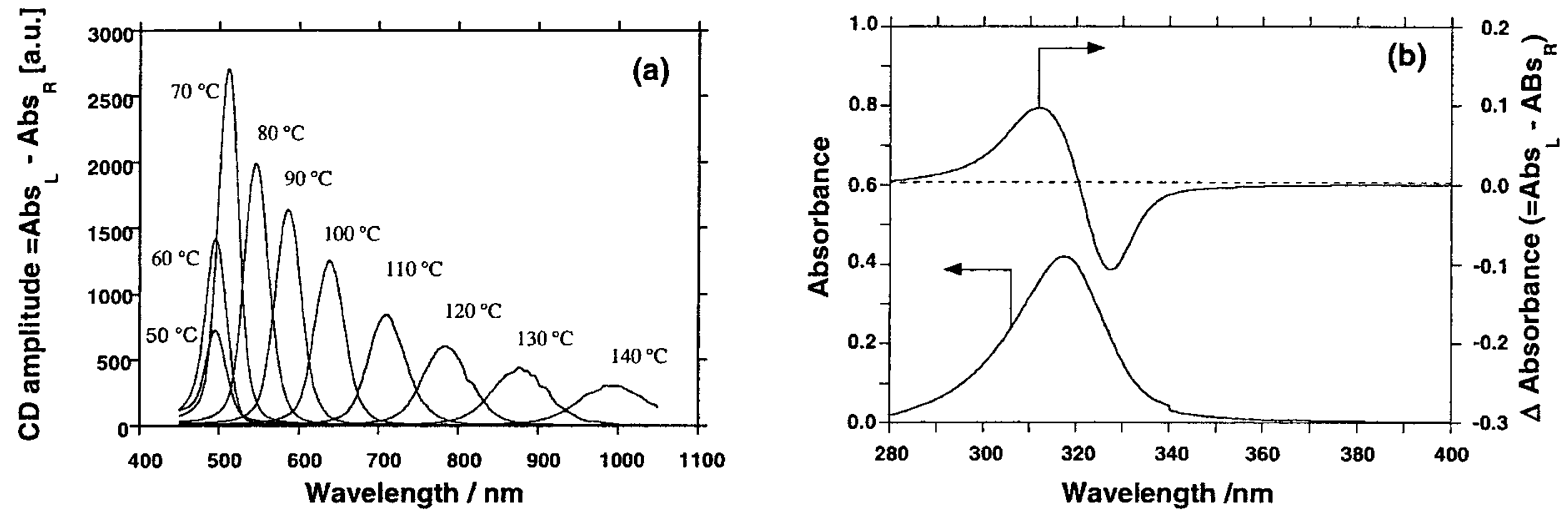

Figure 19. (a) $\mathrm{CD}$ reflection bands of 6 shown by cholesteric phases at various temperatures on cooling run $\left(M_{\mathrm{w}}=11100, \mathrm{DP}_{\mathrm{w}}=46\right.$, and $M_{\mathrm{w}} / M_{\mathrm{n}}=1.19$ ) and (b) $\mathrm{UV}$ and $\mathrm{CD}$ absorption signal intensity at $80^{\circ} \mathrm{C}$.

low $70^{\circ} \mathrm{C}$ in the cooling run from $120^{\circ} \mathrm{C}$ is insensitive to change in temperature and the intensity of the $\mathrm{CD}$ reflection band in visible region decreases markedly.

Polysilane 6 in the TChLC phase at $80^{\circ} \mathrm{C}$ exhibits a UV band at $318 \mathrm{~nm}$, and a bisignate $\mathrm{CD}$ band with equal rotational strengths with extrema at 313 and $327 \mathrm{~nm}$, respectively, as shown in Figure 19b. The bisignate $\mathrm{CD}$ band results from the couplet $\mathrm{CD}$ signals of a chirally ordered motif between two interacting excited states of adjacent $\mathbf{6}$ molecules in the cholesteric helical environment. These considerations led to the idea that the chiral motif takes an $M$ helicity on the basis of the exciton couplet assignment, which is consistent with the assignment of $M$-helicity to the TChLC phase above $70^{\circ} \mathrm{C}$. On the assumption that $\lambda=n_{\mathrm{av}} \times p, \Delta \lambda=\lambda \Delta n / n_{\mathrm{av}}$, and $n_{\mathrm{av}}=2$ for $\mathbf{6}$, while $\lambda$ is reflection wavelength in $\mathrm{nm}, p$ pitch, $n_{\mathrm{av}}$ average refractive index, $\Delta \lambda$ bandwidth, $\Delta n$ difference in refractive index between extraordinary and ordinary light, approximately 175 and 350 molecules of 6 at 70 and $120^{\circ} \mathrm{C}$, respectively, are included per half-pitch in the TChLC phase.

The $M$-cholesteric pitch quickly altered in response to the change in temperature with little hysteresis on heating and cooling runs. On fast cooling, the recovery time of the cholesteric pitch was less than 1-2 min. The absence of intense forces, such as hydrogen bonding, dipole-dipole, or $\pi-\pi$ interactions, and the existence of very weak van der Waals interaction might be responsible for the unique TChLC characteristics. These quick and no-hysteresis response CD band characteristics in the visible and near infrared regions are indeed useful in a practical-level circular polarizing band-path filter including blue, green, red, and black color. These characteristics could be applied in the areas of display, thermometer, and optical fiber communication technologies. These findings may open fresh perspectives for achieving a practical level chiral polysilane-based ap- plication, which avoids one of the major problems of polysilanes, which is main chain scissoring by UV light irradiation.

Rod-like 6 adopting an almost $7_{3}$ helical conformation belongs to a unique set of stiff polymers, exhibiting both $\mathrm{TChLC}^{30 \mathrm{n}}$ and lyotropic liquid crystallinity. ${ }^{30 \mathrm{r}} \mathrm{In}-$ deed, we found that solutions of $\mathbf{6}$ became choresteric at high concentrations. ${ }^{30 \mathrm{r}}$ It was shown that the isotropicbiphasic phase boundary concentration increases as the molecular weight is increased. This increase has been described theoretically using the molecular parameters determined from dilute solution data.

\section{OPTICALLY ACTIVE POLY(DIALKOXYSILANE)S}

\section{The First Synthesis and UV/CD Spectra of Poly(dialkoxysilane)s}

Linear high molecular weight $\left(\mathrm{SiH}_{2}\right)_{n}$, one of the most fundamental model compounds for polysilanes, is still not synthesized, and although a few theoretical studies have been reported for this and some other heteroatom polysilanes, there are almost no experimental data concerning the effect of heteroatoms on the main chain. ${ }^{65}$ Recent theoretical results for conjugated silicon chains bearing oxygen substituents indicate a reduction of the optical band gap as a result of silicon $\sigma$ oxygen $n$ mixing. The main reason for this lack of experimental data may be the difficulty of access to such heteratom-substituted polysilanes. The most widely used synthetic procedure is the Wurtz-type reductive coupling of dichlorosilanes mediated by sodium in refluxing toluene, as stated in Chart 2. However, these reaction conditions severely limit side chains to essentially saturated alkyl or aryl groups. Although the masked disilene approach was applied to the synthesis of certain amino-substituted polysilanes, this method was not successful in the case of alkoxy-substitution. ${ }^{38 c}$ 
There are several reports describing partial heteroatom functionalization by replacement of aryl groups or $\mathrm{H}$, though the degree of substitution usually ranges from 50 to $80 \% .{ }^{66}$ Recently, virtually complete chlorodearylation of poly(aryl-alkylsilane)s has been demonstrated using acetyl chloride/aluminum chloride and the resulting poly(alkyl-chlorosilane)s were successfully substituted with alcohols, amines and mercaptans, yielding polymers with one heteroatom per repeat unit. ${ }^{67}$ Concerning poly[bis(heteroatom)silane]s, there is only one example, poly(dimethoxysilane) $\left(\mathbf{3 2}, M_{\mathrm{w}}=\right.$ $45000, \lambda_{\max }$ at $332 \mathrm{~nm}$ ) synthesized by the anionic ringopening polymerization (ROP) of octamethoxycyclotetrasilane. ${ }^{68}$

Recently, a new synthetic route to several bisheteroatom-substituted polysilanes, poly(dialkoxysilane)s, was reported, as shown in Chart 7.

It was already found that UV light-promoted ROP of octachlorocyclotetrasilane single crystal produces perchloropolysilane, $\left(\mathrm{SiCl}_{2}\right)_{n}(\mathbf{3 3})$, which is an almost insoluble, off-white solid material, is highly reactive. ${ }^{69}$ Reaction of this material with a number of alcohols in the presence of an amine afforded poly(dialkoxysilane)s (inter alia $\mathbf{3 4}, \mathbf{3 5}$, and 36) with moderate molecular weight and permitted the study of the spectroscopic properties of the resulting soluble polymers.

As is evident in Figure 20a, 35 in isooctane at room temperature exhibits a UV absorption due to the main chain $\sigma-\sigma^{*}$ transition at $347 \mathrm{~nm}$ with a fwhm of $23 \mathrm{~nm}$ and shows FL emission at $368 \mathrm{~nm}$ with a Stokes shift of $21 \mathrm{~nm}$, and an associated weak broad emission at $496 \mathrm{~nm}$. Takeda et al. calculated the electronic band structures of several forms of siloxene, one of which was based on a linear chain model which comprises

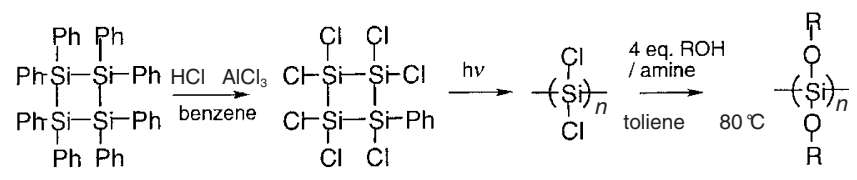

Chart 7. Synthesis of optically active polysilanes bearing alkoxy side groups. polysilane linear chains with $\mathrm{Si}-\mathrm{O}-\mathrm{Si}$ cross-links between them. ${ }^{71}$ The band structure renders possible two optical transitions: a direct-gap and also an indirectgap transition. Thus, dual emission is possible from oxy-substituted polymeric silicon species (though very weak from the indirect-gap). Therefore, the broad long wavelength emission observed for $\mathbf{3 5}$ could indicate $\mathrm{Si}-$ $\mathrm{O}-\mathrm{Si}$ cross-links, presumably due to slight hydrolysis of Si-OR groups and cross-linking at silicons bearing hydroxy groups. The FLA around the 323-nm UV$\mathrm{CD}$ bands tends to approach the theoretical limit of 0.4 expected for the random distribution of a rod chromophore being collinear with the fluorophore in a rigid medium.

As demonstrated in Figure 20b, optical activity of $\mathbf{3 5}$ is evidenced by the observation of a positive Cotton effect in the CD spectrum, which is coincident with the UV absorption due to the silicon main chain $\sigma-\sigma^{*}$ transition. Cooperative interaction between the enantiopure chiral alkoxy side chains induces a preferential screw sense helical order. The Kuhn disymmetry ratio $g_{a b s}$ for 35 at $-70^{\circ} \mathrm{C}$ is $1.6 \times 10^{-4}$. In the case of poly [ $n$-alkyl(S)-2-methylbutylsilane]s, the $g_{\text {abs }}$ value of $1.9 \times 10^{-4}$ may correspond to a single screw sense helical polysilane main chain. It is likely, therefore, that the main chain of $\mathbf{3 5}$ adopts helical segments of predominantly either $P$ - or $M$-screw sense. In contrast to the positive Cotton effect for $\mathbf{3 5}$, polysilane $\mathbf{3 6}$ bearing $(S)$-2methylbutoxyl side chains, shows a negative Cotton effect at $-10^{\circ} \mathrm{C}$, indicating a preferential, but opposite, screw sense in $\mathbf{3 5}$.

The idea that poly(dialkoxysilane)s adopt helical main chain conformations is supported by molecular mechanics calculations for the model polymer, $\mathrm{H}_{-}$ $\left[\mathrm{Si}(\mathrm{O}-n-\mathrm{Bu})_{2}\right]_{30}-\mathrm{H}(37)$, which indicate two local energy minima at dihedral angles of 160 and $200^{\circ}$ (see Figure 21c), i.e., these polymers adopt helical main chain structures, of between approximately $7_{3}$ and $15_{7}$ screw pitch ( $\left.c a .47_{21}\right)$, but are optically inactive due to equal proportions of $P$ - and $M$ - turns. Interestingly, molecular mechanics calculations for $\mathrm{Cl}-\left(\mathrm{SiCl}_{2}\right)_{30}-\mathrm{Cl}$

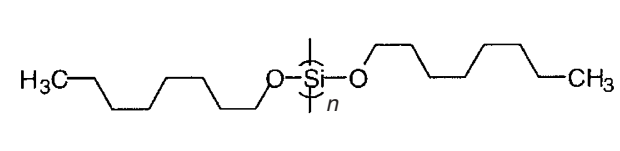

34

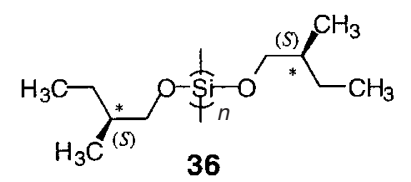

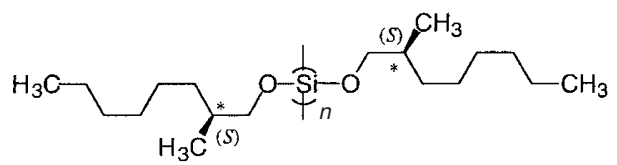

35

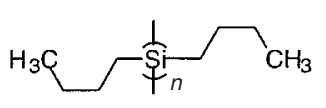

14

Chart 8. Chemical structures of poly(dialkoxylsilane)s (34-36) and poly(di- $n$-butylsilane) (14). 

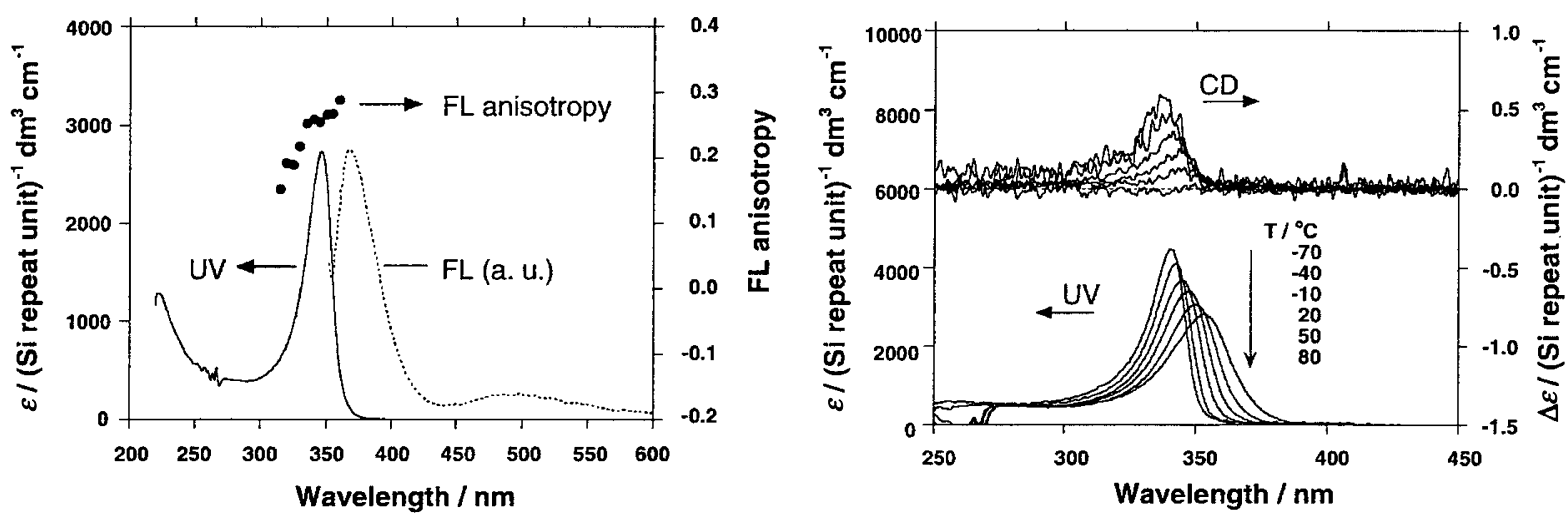

Figure 20. (a) Variable temperature UV and CD spectra of $35\left(M_{\mathrm{w}}=33000\right.$ and $\left.M_{\mathrm{n}}=15000\right)$ in isooctane.
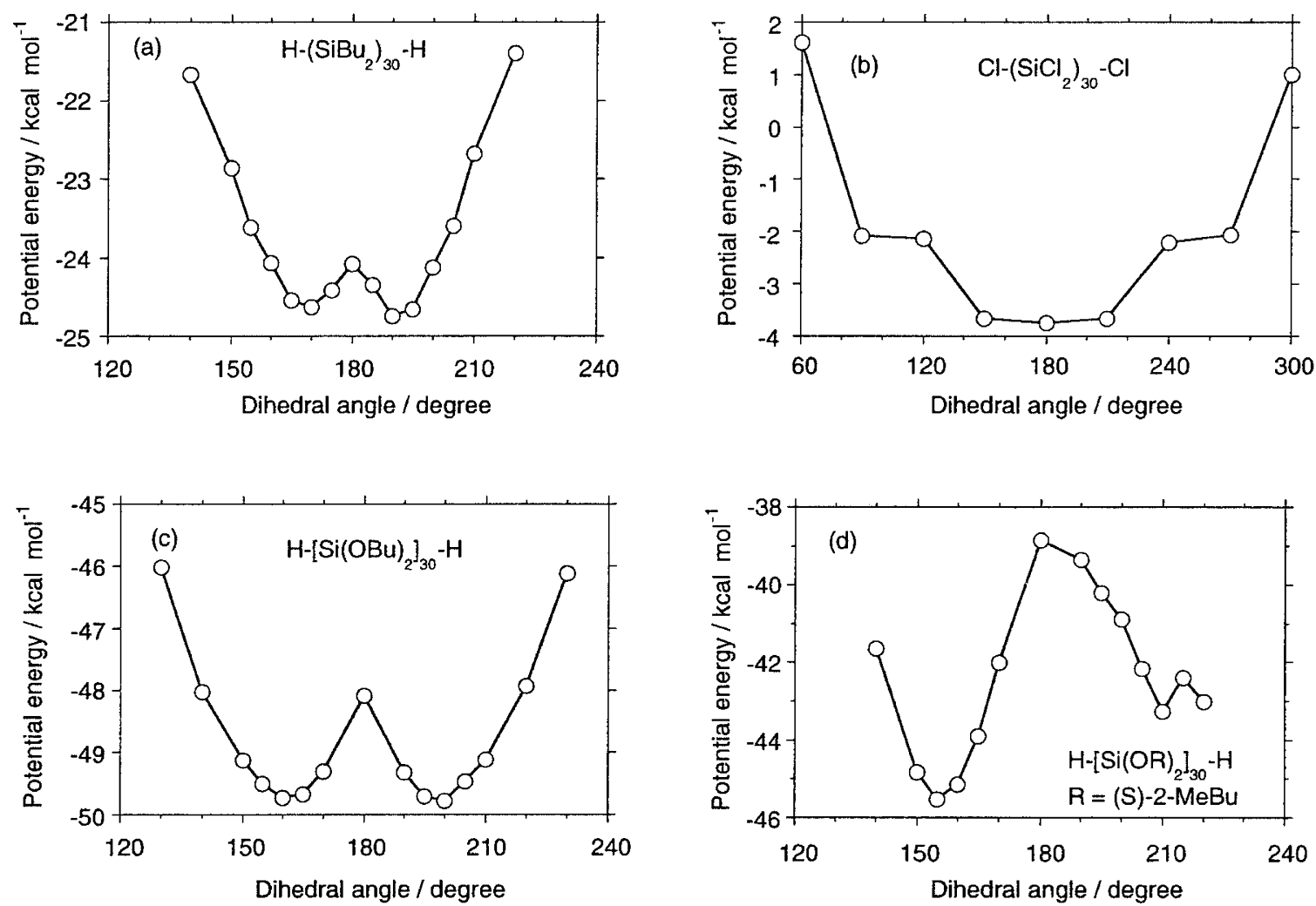

Figure 21. Molecular mechanics calculation. Main chain dihedral angle dependence of the potential energy of (a) di- $n$-butylsilane thirty repeat units with hydrogen termini (oligo-14), (b) $\left(\mathrm{SiCl}_{2}\right)$ thirty repeat units with chlorine termini (oligo-33), (c) di- $n$-butoxysilane thirty repeat units with hydrogen termini (oligo-37), and (d) di-(S)-2-methylbutoxysilane thirty repeat units with hydrogen termini (oligo-36).

favour the all-anti conformation at $180^{\circ}$ as a minimum energy, as seen in Figure $21 \mathrm{~b}$, in aggreement with the reported single crystal X-ray structure. ${ }^{69}$

\section{Energy Curves of Poly(dialkoxysilane)s}

To allow comparison of alkoxy-and alkyl-substituted polymers , molecular mechanics calculations were also performed for poly(di- $n$-butylsilane), 14. The results indicated two local energy minima at dihedral angles of 165 and $205^{\circ}$, as seen in Figure 21a, though the experimentally determined dihedral angles are $\sim 154$ and $206^{\circ}$ at room temperature in solid state. For the model polymer for 36, $\mathrm{H}-\left[\mathrm{Si}\left(\mathrm{O}-(S)-2 \mathrm{MeBu}_{2}\right)_{30}-\mathrm{H}\right.$, contain- ing enantiopure chiral alkoxy side chains, calculations afford a markedly asymmetric potential energy plot, as seen in Figure 21d, indicating a preference for $P$-screw sense (dihedral angle $155^{\circ}$ ) over $M$-screw sense, consistent with the observed optical activity.

\section{Evidence for Silicon $\sigma$-Oxygen $n$ Mixing Interaction in Poly(dialkoxylsilane)s}

The UV absorption wavelength maxima for the poly(dialkoxysilane)s are generally red-shifted by $\sim 20 \mathrm{~nm}$ compared to their symmetrical poly(dialkylsilane) analogs. Such bathochromic shifts upon substitution of $\mathrm{C}$ with $\mathrm{O}$ on a silicon main chain have been 
reported in poly[alkoxy(phenyl)silane]s. ${ }^{67}$ The UV absorption maxima of the alkoxy-substituted polymers were found to be red-shifted about $26 \mathrm{~nm}$ relative to the poly[alkyl-(phenyl)silane]. ${ }^{32 f}$ The reason for this was discussed in terms of the interaction between the lone pair of electrons on the oxygen substituent and the polymer main chain electronic structure, resulting in destabilization of the HOMO and concomitant decrease of the transition energy. ${ }^{65}$ Experimental results in the poly(dialkoxysilane) study are consistent with the band theory which predicts a bathochromic shift due to $\mathrm{Si}$ $\sigma$-oxygen $n$ interaction. From the theory along with the molecular mechanics calculations, we conclude that the origin of the bathochromically shifted UV absorption in poly(dialkoxysilane)s compared to poly(dialkysilane)s is indeed electronic, through silicon $\sigma$-oxygen $n$ orbital interaction, and not steric. ${ }^{34}$

\section{Hypsochromic Thermochromism}

Certain poly(di- $n$-alkylsilane)s exhibit abrupt thermochromism at lower temperatures with the appearance of a sharper, longer wavelength absorption around $354 \mathrm{~nm}$, the driving force of which has been attributed to both a single chain mechanism and conformationdependent polymer-solvent dispersion interactions. Also poly(n-alkylmethylsilane)s, such as $\operatorname{poly}(n-$ propylmethylsilane) and poly(n-hexylmethylsilane), show smooth bathochromic shifts without abrupt transition, attributed to a continuously increasing relative population of extended main chain sections with cooling. ${ }^{39}$

In contrast, for poly(dialkoxysilane)s 34-36, such bathochromic spectral shifts were not observed on cooling. The UV and CD absorption maxima for $\mathbf{3 5}$ undergo small monotonic hypsochromic shifts as the temperature is reduced from 80 to $-70^{\circ} \mathrm{C}$ without any abrupt changes. This behavior is similar to that observed for stiff poly(diarylsilane) $\mathrm{s}^{3 \mathrm{c}}$ and $\beta$-branched rod-like poly(dialkylsilane)s bearing $\beta$-branched side chains. ${ }^{30 \mathrm{k}}$

\section{OPTICALLY ACTIVE POLY[ALKYL(ALKOXYPHENYL)SILANE]S}

\section{Substituent Effects on Optical Properties and Global- Local Conformations}

The conformational structures of poly(dialkylsilane) main chains at the macro- and microscopic levels, and their optoelectronic properties, dependent upon the optical band gap, are successfully controllable by choice of the side chain structures. ${ }^{35 a, 35 b}$ In the case of phenyl-substituted polysilanes, electronic interaction between the delocalized silicon chain $\sigma$-bonding or-
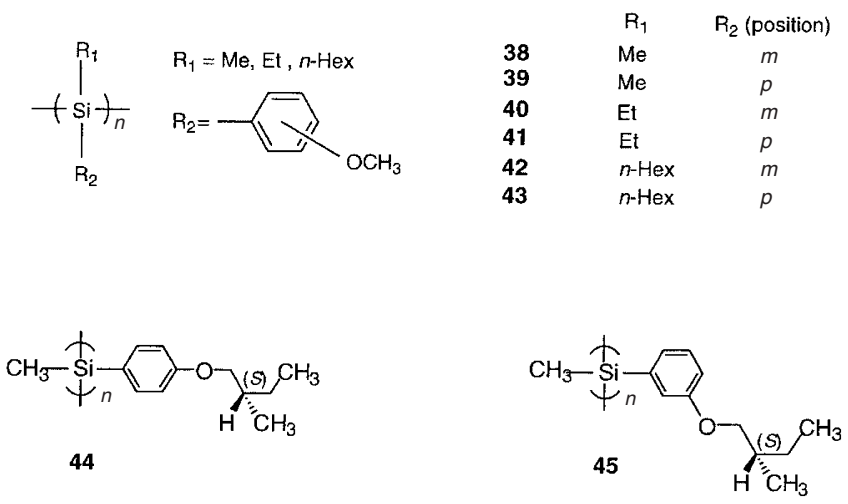

Chart 9. Chemical structures of poly[alkyl(alkoxyphenyl)silane]s (38-45).

bitals and the $\pi$-orbitals of the aryl groups causes a significant modification of the band gap and conformational properties. ${ }^{35 \mathrm{c}, 65}$ These aryl-substituted polysilanes may be potential candidates for applications in a molecular-based chiroptical switch and memory in the UV/visible region. On the other hand, the precise control of helical polymers is of great interest, due to the technological importance of polymeric materials in the analysis and preparation of chiral drugs. ${ }^{70}$ Indeed, a few synthetic helical polymers are able to be exploited as chiral selectors for a particular chiral drug. Aiming at chiral selection, it is necessary to introduce certain functional groups capable of discriminating between a pair of guest enantiomers through intermolecular interactions such as hydrogen bonding, phenyl $\pi-\pi$, dipole-dipole, ionic, and acid-base interactions. Helical poly[alkyl(alkoxyphenyl)silane]s with chiral side chains may be candidates for use as such a chiral selector due to their multiple chiral recognition sites such as aryl, oxygen, and enantiopure chiral group functionalities. ${ }^{32 \mathrm{e}, 31 \mathrm{~g}}$

The absorption spectra of poly[alkyl(alkoxyphenyl)silane]s are susceptible to substituent effects such as charge transfer from the phenyl ring to the silicon main chain ( $\sigma \pi$-mixing), the position of the electrondonating alkoxy group on the phenyl ring, or the introduction of enantiopure chiral groups. ${ }^{32 c, 32 e, 32 g, 73,74} \mathrm{We}$ systematically investigated the UV absorption properties in THF at $20^{\circ} \mathrm{C}$ of a series of optically inactive poly[alkyl(methoxyphenyl)silane]s (38-43) with different degrees of chain coiling, which are controlled by the alkyl substituents and the position of the methoxy group on the phenyl ring, ${ }^{74}$ as shown in Chart 9 .

As shown in Figure 22, the absorption properties of 38-43 greatly depend on the global shape (from a shrunken coil to an extended stiffer structure on increase of the alkyl side chain length) and also the positional effect of the alkoxy moiety in the phenyl ring ( $m$ - or $p$-position). Additionally, incorporation of $20 \%$ 
enantiopure chiral silane units into these polysilanes led to optically active helical copolymers, which exhibited "Sergeants and Soldiers"-type cooperativity.

\section{Control of Main Chain Helicity by Position of Remote Chiral Group}

The helical structures of poly[alkyl(alkoxyphenyl)silane]s featuring a remote enantiopure chiral group on the phenyl ring are significantly affected by the position (meta- or para-) of the chiral alkoxy substituent, even though the chiral substituent is far from the main chain. ${ }^{32 \mathrm{c}}$ As shown in Figure 23a, the CD spectrum of the $m$-derivative (45) shows a marked negative signal at around $310 \mathrm{~nm}$ in the region between the two UV bands, indicating that the chiral steric effect locks the main chain conformation in a particular helical sense, even though it may be strongly distorted due to the steric imbalance between the methyl and bulky $m-(S)$ 2-methylbutoxyphenyl side chains.

Contrarily, in the case of the $p$-derivative (44), no $\mathrm{CD}$ bands were observed at $23-25^{\circ} \mathrm{C}$, even at $-80^{\circ} \mathrm{C}$, as shown in Figure 23b. Presumably the Cotton band of the $p$-derivative is cancelled by an equivalent pro-

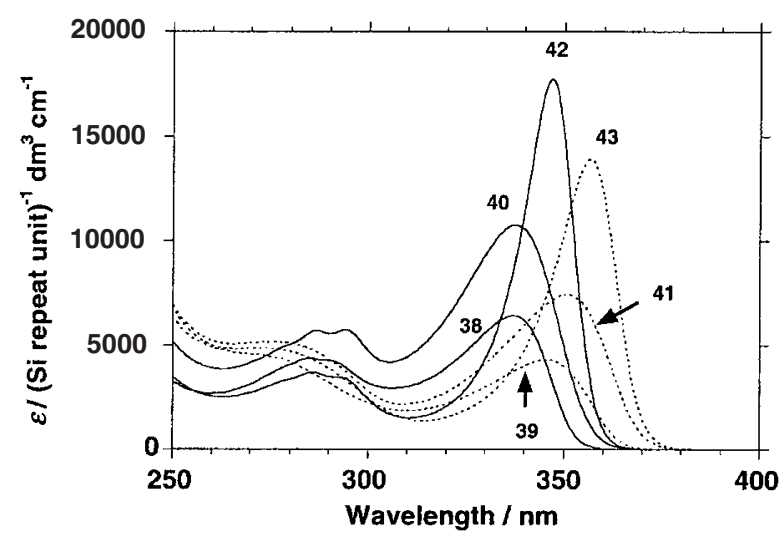

Figure 22. UV spectra of poly[alkyl(methoxyphenyl)silane] derivatives 38-43 in THF at $20^{\circ} \mathrm{C}$. Viscosity indices in THF at $30^{\circ} \mathrm{C}$ are $\alpha=0.45$ for 38, $\alpha=0.35$ for 39, $\alpha=0.63$ for $\mathbf{4 0}, \alpha=$ 0.64 for $\mathbf{4 1}, \alpha=0.96$ for $\mathbf{4 2}$, and $\alpha=0.94$ for $\mathbf{4 3}$.

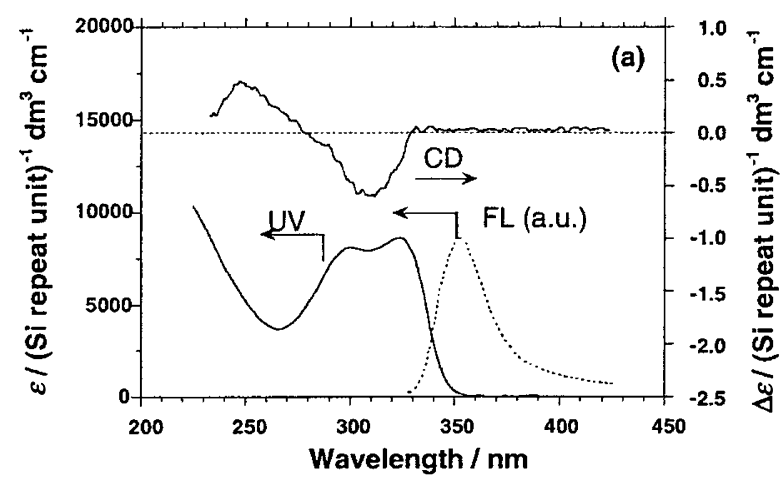

portion of opposite screw-sense helical segments (this may be referred to as "latent helicity", "masked CD", or "crypto-optical activity"). ${ }^{50}$ The difference between the higher order structures of the $m$ - and $p$-derivatives may be connected to the different degrees of steric hindrance of the $(S)$-2-methylbutoxyphenyl rings in para and meta positions, since the $p$-derivative may have more space in which to freely rotate the aryl group, and as a result, the main chain is not fixed conformationally.

The difference in the helical main chain mobilities of 44 and $\mathbf{4 5}$ are evident in their ${ }^{29} \mathrm{Si}$ NMR spectra, ${ }^{32 \mathrm{c}}$ as shown in Figure 24. A broad signal $\left(\Delta v_{1 / 2} \sim 240 \mathrm{~Hz}\right.$ at around $-32 \mathrm{ppm}$ ) is observed for optically active 45, whereas two sharp signals (at about $-39 \mathrm{ppm}$ and $-40 \mathrm{ppm}, \Delta v_{1 / 2}$ about $76 \mathrm{~Hz}$ and $85 \mathrm{~Hz}$, respectively) and one shoulder (at about $-38 \mathrm{ppm}$ ) for optically inactive $\mathbf{4 4}$ are observed. The two sharp signals of $\mathbf{4 4}$ may reflect the tacticity of the main chain with $s t-$, it-, and $h t$ - triads. ${ }^{72}$ The broad signal of $\mathbf{4 5}$ suggests that the mobility of the main chain is restrained due to chiral steric locking in one predominant helical screw-sense. In contrast, the sharpness of the NMR signal of $\mathbf{4 4}$ indicates that the $P$ - and $M$-helical segments in the main

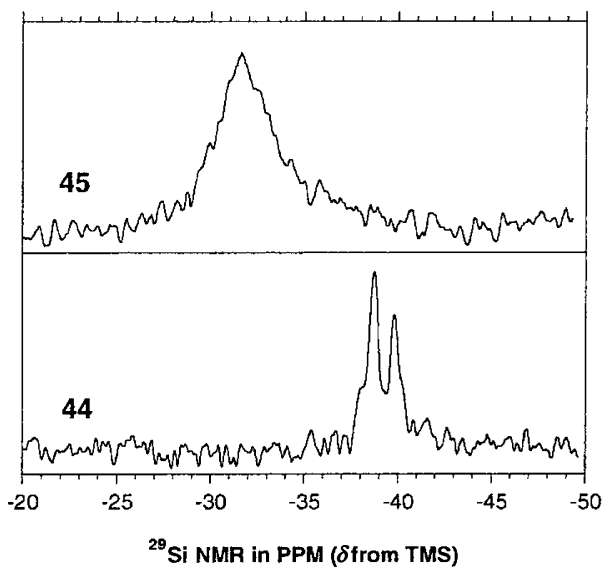

Figure 24. ${ }^{29} \mathrm{Si}$ NMR spectra of para and meta poly$\{$ methyl $[(S)-2$-methylbutoxyphenyl]silane $\}$ s $(44$ and 45$)$ in benzene- $d_{6}$ at $50{ }^{\circ} \mathrm{C}$.

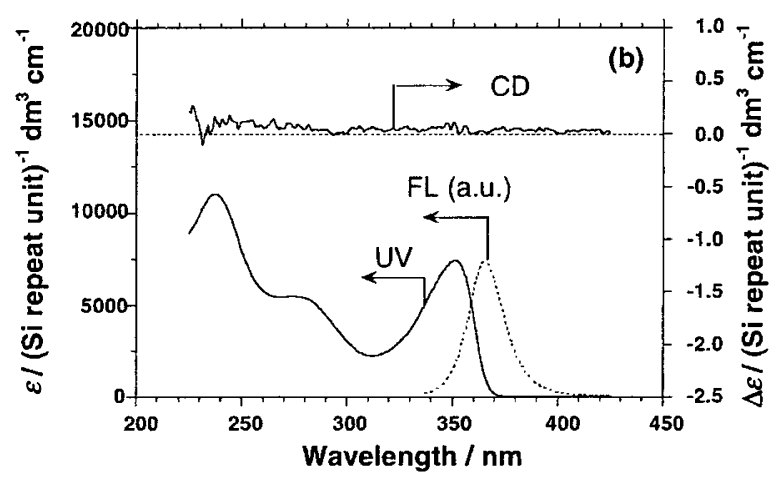

Figure 23. $\mathrm{UV}, \mathrm{CD}$, and FL spectra of (a) poly\{methyl $[m-(S)-2$-methylbutoxyphenyl $]$ silane $\}$ (45) and (b) poly\{methyl $[p-(S)-2-$ methylbutoxyphenyl]silane $\}$ (44) in THF at $23-25^{\circ} \mathrm{C}$. 

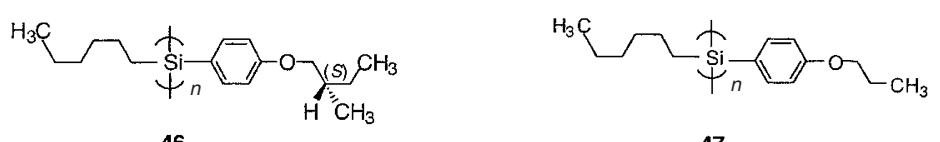

46

47

Chart 10. Chemical structures of poly[alkyl(alkoxyphenyl)silane]s (46 and 47).
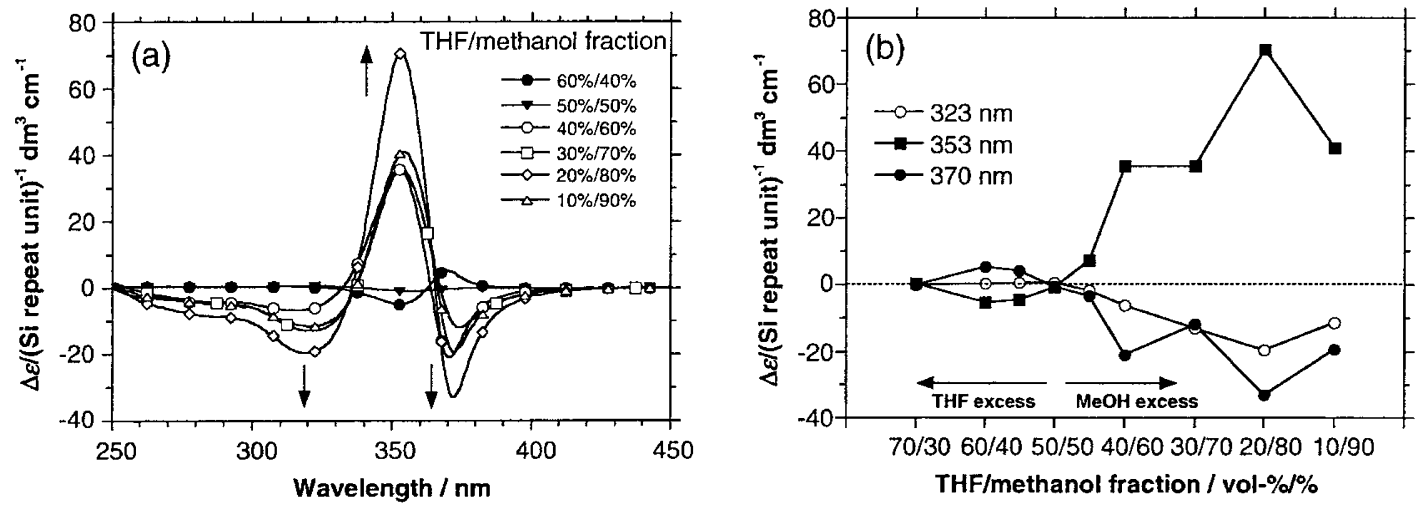

Figure 25. (a) CD spectra and (b) plot of CD amplitudes of poly $\{n$-hexyl[ $p$ - $(S)-2$-methylbutoxyphenyl $]$ silane $\}$ (46) aggregates at different $\mathrm{THF} /$ methanol proportions at $20^{\circ} \mathrm{C}$.

chain can rapidly exchange without locking into a partial screw-sense. In support of this, it was recently reported that the ${ }^{1} \mathrm{H}$ NMR linewidths of poly(alkyl isocyanate)s are directly related to the degrees of mobilities of the main chain, side chains, and interconversion between $P$ - and $M$-screw senses. ${ }^{18 \mathrm{r}}$

\section{Chiroptical Properties with Switch and Memory Func-} tions of Aggregates

As noted above, poly[alkyl(alkoxyphenyl)silane]s bearing enantiopure chiral substituents in the para position on the phenyl ring are optically inactive in dilute solution due to the presence of equal proportions of $P$ - and $M$-helical segments. ${ }^{31 c, 31 \mathrm{e}}$ However, it is well known for several optically inactive $\pi$-conjugated polymers bearing chiral side chains that optical activity may emerged in the aggregate phase due to supramolecular ordering. Interestingly, aggregation of polymers with chiral substituents into chiral supramolecular structures considerably influences the optical and chiroptical properties. For instance, the bisignate CD signal switching of polythiophene $e^{20 \mathrm{c}, 20 \mathrm{~d}, 20 \mathrm{~g}, 20 \mathrm{i}}$ or $\operatorname{poly}(l-$ aspartate $)^{55}$ aggregates and/or liquid crystals in response to the cooling rate and/or temperature of the thin film or the ratio of good/poor co-solvents have been reported. Poly[alkyl(alkoxyphenyl)silane]s aggregates also show switchable and memorizable CD properties in good/poor cosolvent systems, controllable by such experimental factors as solvent polarity, solvation, solubility, solvent addition order, thermal effects and polysilane structure and stereochemistry. ${ }^{31 \mathrm{e}}$

The CD spectra of poly $\{n$-hexyl $[p-(S)-2$-methylbutoxyphenyl]silane (46) aggregates exhibit a wellresolved bisignate $\mathrm{CD}$ signal in a $\mathrm{THF} /$ methanol co- solvent system, although no CD signal is evident in pure THF solution. ${ }^{31 \mathrm{e}}$ The observed positive Cotton effect in excess THF inverted to a negative Cotton effect above $60 \%$ methanol, and the bisignate $\mathrm{CD}$ intensities changed continuously with increasing methanol concentration (Figure 25a), the CD inversion occuring at $50 \% / 50 \% \mathrm{THF} / \mathrm{methanol}$. This indicates that although 46 forms optically active chiral aggregates in a poorer (high dielectric constant) solvent, the chirality of the aggregates is switchable by the solvent polarity, as has also been reported for a polythiophene. ${ }^{20 \mathrm{~d}}$ The bisignate nature of the $\mathrm{CD}$ spectrum is considered to be characteristic of an exciton couplet arising from the interaction between transition dipole moments on neighboring polymer segments in chiral configurations. ${ }^{43}$ The CD splitting results mainly from intermolecular interaction between polysilane helices since the CD spectra originate from $\mu \mathrm{m}$-scale chiral aggregates. ${ }^{31 \mathrm{e}}$

Interestingly, the $\mathrm{CD}$ sign of the chiral aggregates around the $\mathrm{CD}$ reversal point (THF/methanol $55 \% / 45 \%$ ) was found to be switchable by the solvent addition order, either Method I (methanol added into THF solution including the polysilane) or Method II (THF solution of the polysilane added into methanol), as shown in Figure 26. This CD inversion is assumed to originate from the initial chirality of the "seed" aggregates, dependent on the solvent polarity in the aggregation process.

\section{Temperature and Solvent Effects on Chiroptical Switch} and Memory

Thermally reversible aggregation and gelation have been reported for stiff $\operatorname{poly}(n$-hexyl 
isocyanate)s. ${ }^{18 b, 18 j}$ To test the thermal stability of the chirality of optically active poly $\{n$-hexyl- $[p$ $(S)$-2-methylbutoxyphenyl]silane $\}$ aggregates, $\mathbf{4 6}$ aggregates were subjected to multiple cycles of heating $\left(50^{\circ} \mathrm{C}\right)$ and cooling $\left(-10^{\circ} \mathrm{C}\right){ }^{31 \mathrm{e}}$ In the $\mathrm{CD}$ spectra of the aggregates at $60 \% / 40 \% \mathrm{THF} /$ methanol, the bisignate Cotton effect observed at $20^{\circ} \mathrm{C}$ completely disappeared at $50^{\circ} \mathrm{C}$ and appeared again at $-10^{\circ} \mathrm{C}$ on the cooling run with similar $\lambda_{\text {ext }}$ and sign to that at $20^{\circ} \mathrm{C}$ (Figure 27a). These CD profiles are reproducible after several thermal cycles. In contrast, at $50 \% / 50 \%$ $\mathrm{THF} /$ methanol (CD reversal point), the CD spectra of the aggregates for only Method II (THF solution of 46 added into methanol) reversed after four thermal cycles and the CD profiles finally showed similar CD $\lambda_{\text {ext }}$, signs and intensities to those obtained by Method $I$ (Figure 27b). This CD switching for Method II results from the transformation of a metastable chiral aggregate to an energetically more stable opposite chiral state (produced using Method I) on application of the thermal stimulus.

The formation of stable chiral aggregates depends

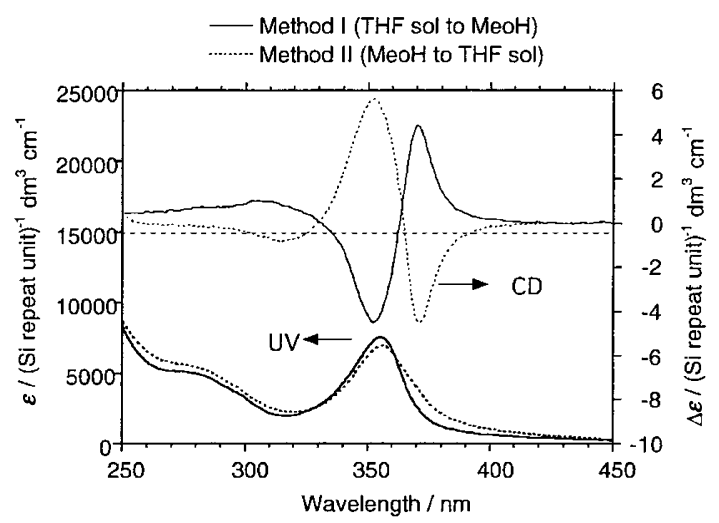

Figure 26. UV-visible and CD spectra of $\operatorname{poly}\{n$-hexyl $[p-(S)$ 2-methylbutoxyphenyl]silane $\}$ (46) aggregates prepared by Methods I and II at $55 \% / 45 \% \mathrm{THF} /$ methanol volume ratio at $20^{\circ} \mathrm{C}$.

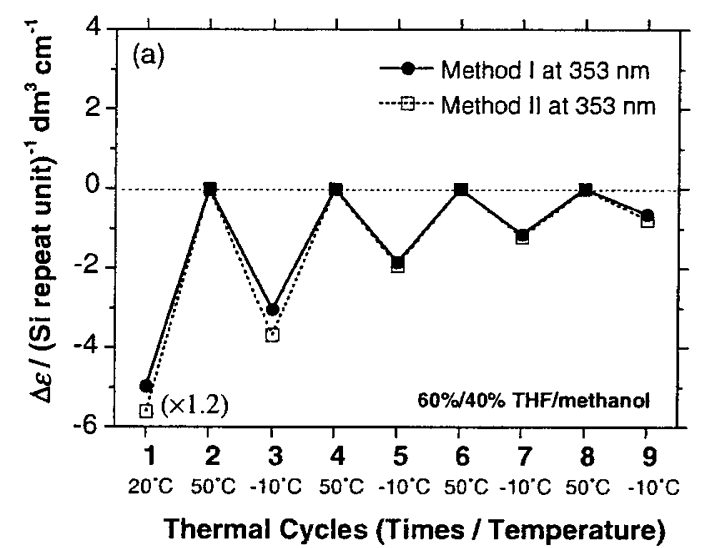

critically on the polysilane structure and stereochemistry. The less sterically hindered $p$-substituted 44 exhibits a weak, bisignate, non-switchable CD signal in only the toluene/acetonitrile system, and no CD signals are evident in pure toluene or THF due to the lack of preferential screw sense. In contrast, although the apparently less sterically hindered, less polar $m$ substituted $\mathbf{4 5}$ does show optical activity when molecularly dispersed in THF or toluene solution, the CD signal disappears on formation of aggregates in good/poor cosolvent systems. These methyl-substituted polysilanes have sterically imbalanced side chains (small methyl group and bulky (S)-2-methylbutoxyphenyl group) and may adopt conformations with macroscopically random and shrunken coils. In contrast, the stiffer 46 adopts a more extended global shape with greater spatial freedom between the bulky side chains, leading to the formation of well-balanced, chirally oriented aggregates and the chirality switching function.

\section{Molecular Chirality Recognition Abilities of Aggre- gates}

A few synthetic helical polymers are well known to be utilized as chiral selectors and chiral stationary phases (CSP) in gas or liquid chromatography. ${ }^{70}$ Recently, it was reported that a preferential helical sense in solution can be induced by interaction between optically inactive polymers and chiral solvents/additives. Examples of such intriguing polymers are poly $(n$-hexyl isocyanate $)^{18 \mathrm{~d}, 18 \mathrm{k}, 75}$ and poly(phenylacetylene)s bearing functional groups. ${ }^{19 \mathrm{~d}-19 \mathrm{~h}}$ Certain polysilane derivatives also show chiral recognition ability in solution at room temperature. Poly(methyl- $\beta$-pinanylsilane) (48) has two chiral centers per bulky hydrophobic pinanyl side group ${ }^{30 \mathrm{i}}$ and exhibits an enantio-recognition ability for hydrophobic $\beta$-pinene, but none for hydrophilic methanol.

We also demonstrated the molecular chiral-

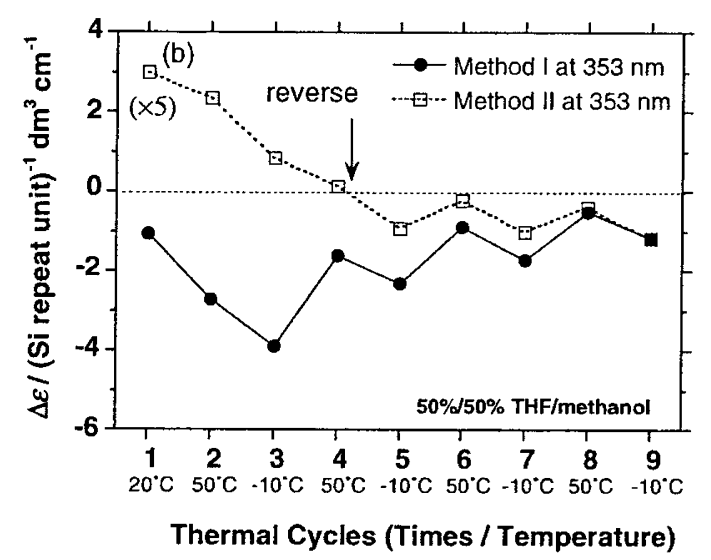

Figure 27. Changes of CD intensity of poly $\{n$-hexyl[ $p$ - $(S)$-2-methylbutoxyphenyl]silane $\}$ (46) aggregates prepared by Methods I and II at (a) $60 \% / 40 \%$ and (b) $55 \% / 45 \% \mathrm{THF} /$ methanol volume ratios in multiple thermal cycles of heating to $50^{\circ} \mathrm{C}$ and cooling to $-10{ }^{\circ} \mathrm{C}$. 

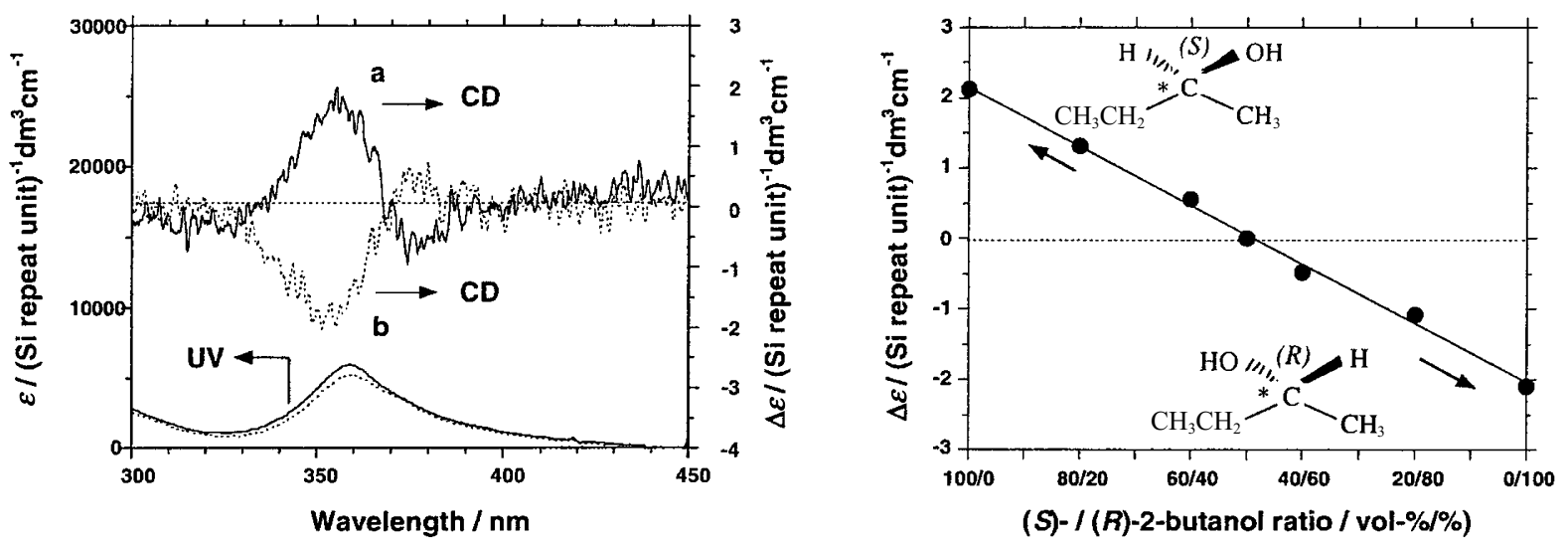

Figure 28. (a) CD and UV spectra of $\operatorname{poly}[n$-hexyl( $p$ - $n$-propoxyphenyl)silane $]$ (47) aggregates in toluene/( $S)$ - (a, solid line) or $(R)-2-$ butanol (b, dotted line)/methanol mixtures at $20^{\circ} \mathrm{C}$ and $(b)$ plot of $\mathrm{CD}$ intensities at $354 \mathrm{~nm}$ of the polysilane aggregates in toluene/various ratio of $(S) /(R)-2$-butanol $/$ methanol mixtures at $20^{\circ} \mathrm{C}$.

ity recognition ability of chiral alcohols using aggregates $^{32 g}$ of optically inactive $\operatorname{poly}[n$-hexyl $(p$ $n$-propoxyphenyl)silane] without any chiral groups (47). Aggregates of $\mathbf{4 7}$ in the presence of various chiral alcohols exhibit remarkable, bisignate induced circular dichroism (ICD) based on amplified chiral ordering as shown in Figure 28a. Additionally, the sign of the ICD depends on the absolute configuration of the chiral alcohol, indicating clearly that the polysilane forms chiral oriented aggregates due to the transfer of molecular chirality information by the weak hydrogen-bonding interaction between the ether oxygen moieties of $\mathbf{4 7}$ and the $\mathrm{OH}$ group of the chiral alcohols. Interestingly, the chiral polysilane aggregates showed a linear relation between the enantiomeric excess (ee) of chiral 2-butanol and the ICD intensities (Figure 28b). This system can thus quantitatively determine the optical purity of chiral target molecules .

Interestingly, in a series of primary $(S)$-chiral alkyl alcohols with monotonically increasing numbers of methylene spacer groups, the ICD sign of the polysilane aggregates was found to oscillate according to the number of methylene carbons between the $\mathrm{OH}$ group and the chiral center (Figure 29).

Such CD phenomena have been generally referred to as the "odd-even" effect and have been observed in other helical superstructures. ${ }^{20 \mathrm{~g}}$ Exciton couplet theory and model studies suggest that the odd-even effect originates in the transition between $P$ - and $M$-handed supramolecular helicity, indicating that the position of the chiral center governs the chirality of the polysilane aggregates. The preparation of a combinatorial library by screening of $\mathbf{4 7}$ aggregates against various chiral guests might help in the design of new polymer-based CSPs or chiral selectors.

The origin of the difference between the optical activities (or lack of it) in 38-47 remains obscure. To the-

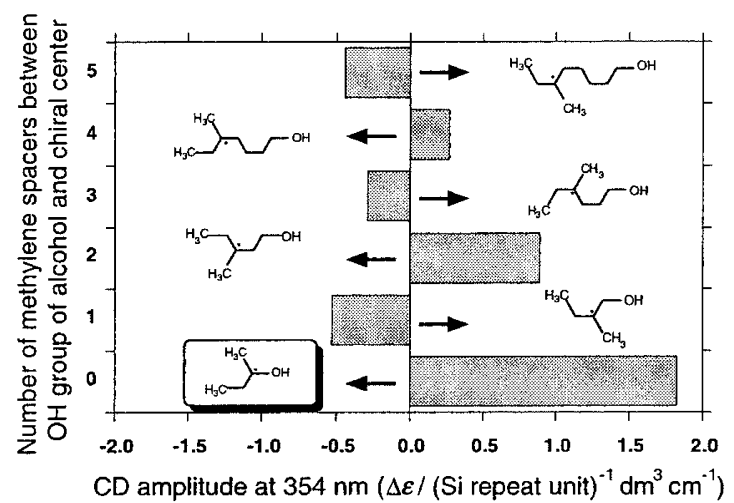

Figure 29. Variation of $\mathrm{CD}$ sign and amplitudes of poly $[n$ hexyl( $p$ - $n$-propoxyphenyl)silane] (47) aggregates in toluene / methanol / a series of $(S)$-primary chiral alkyl alcohols mixtures at $20^{\circ} \mathrm{C}$. For comparison, the $\mathrm{CD}$ intensity with $(S)$-2-butanol is inserted.

oretically clarify this, the potential energy curves of $\mathbf{4 3}$, $\mathbf{4 4}$, and $\mathbf{4 5}$ model oligomers as a function of dihedral angle were obtained by molecular mechanics calculations.

Figure 30a shows the potential energy curves per silicon repeat unit for it-46 and it-47 models with thirty silicon repeat units. Molecular mechanics calculations indicated the existence of two almost equivalent local minima around $165^{\circ}\left(P-15_{7}\right.$ helix $)$ and $195^{\circ}\left(M-15_{7}\right.$ helix) in a double well potential energy plot for it-46 with a very small preferential energy stability between $P$ - and $M$-motifs. In addition, the helix reversal energy of $\sim 2.5 \mathrm{kcal}$ per repeat unit seems to be fairly small, indicating the likelihood of a rapid twisting motion between $P$ - and $M$-motifs. In the case of $i t-47$, the calculations clearly showed two equivalent potential energy minima at $155^{\circ}\left(P-15_{7}\right.$ helix $)$ and $210^{\circ}\left(M-15_{7}\right.$ helix $)$, with a helix reversal of $\sim 2.5 \mathrm{kcal}$ per repeat unit. These results satisfactorily agree with the lack of preferential energy stability between $P$ - and $M$-motifs for $\mathbf{4 7}$ and 

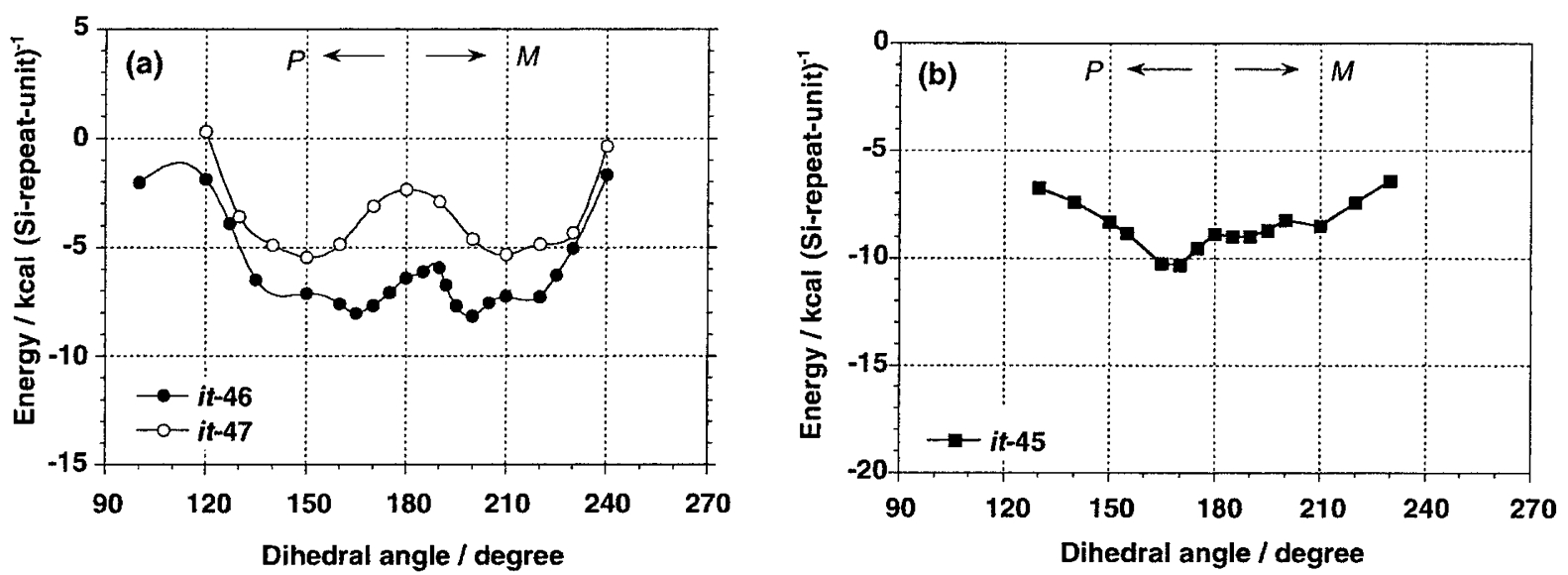

Figure 30. Potential energy of model oligomers per silicon repeat unit of (a) it-46 with thirty silicon repeat units (filled circles) and $i t-47$ with thirty silicon repeat units (open circles), and (b) it-45 with thirty silicon repeat units as a function of the main chain dihedral angle. Calculation was carried out using MSI Discover III ver 4.00 with a PCFF force-field.

lead to the further conclusion that this may be essential for the apparent optical inactivity (masked helicity or cryptochirality) of $\mathbf{4 6}$ and $\mathbf{4 7}$ in solution. Presumably, the optical activity of $\mathbf{4 6}$ may occur in a preferential helical screw sense within the aggregates induced by the $p$-chiral ether moieties, and also the solvent polarity could control the preferential helical screw sense.

For comparison, Figure 30b shows the potential energy curves per silicon repeat unit for an it-45 model with thirty silicon repeat units. Molecular mechanics calculations indicated a local minimum around $165^{\circ}$ in a substantially single well potential energy, resulting in the preferential energy stability of the $P$-motif over the $M$. This result might be connected to the observation of the $\mathrm{CD}$ signal for $\mathbf{4 5}$ in solution at room temperature.

\section{OPTICALLY ACTIVE POLY[ALKYL(PHENYL)SILANE]S}

\section{UV absorption and Fluorescence Spectra of Optically Inactive Homopolymers}

The effect of the cooperative helical ordering in poly[alkyl(alkylphenyl)silane] copolymers with a minor portion of chiral and major portion of achiral moieties is described. ${ }^{31 b, 31 f}$ Firstly, the structure was analyzed to determine the main chain solution state conformation. This is because, although well-established light scattering and viscosity measurements provide information on the global conformation such as the radius of gyration and the value of $\alpha$, these methods cannot definitively determine whether a local conformation is helical. The main chain conformation of poly[alkyl(aryl)silane]s, however, was not fully understood because the main chain $\lambda_{\max }$ depends not only the main chain conformation and the silicon repeat num- ber ${ }^{38}$ but also on the effect of $\sigma-\pi$ mixing between the phenyl groups and the silicon main chain. ${ }^{35}$ Secondly, poly[alkyl(aryl)silane] copolymers with an increasing proportion of chiral side chain to achiral side chains was investigated. ${ }^{31 b, 31 \mathrm{f}}$ The preferential screw-sense in the main chain of these helical polymers was induced nonlinearly as regards the incorporated chiral moiety ratio, which is consistent with a cooperative response of the conformational properties ${ }^{15,18 \mathrm{~b}}$ to the chiral information.

A series of optically active poly[alkyl(phenyl)silane] copolymers with different chiral mole compositions $\mathbf{5 2}$ and 53, are shown in Chart 11, along with homopolymers of coiled poly[methyl(phenyl)silane] (49) and stiff poly $[n$-hexyl( $m$-tolyl)silane] (50).

Figures $31 \mathrm{a}$ and $31 \mathrm{~b}$ compares the UV absorption, FL, and FLA spectra of optically inactive poly[alkyl(alkylphenyl)silane] homopolymers 49 and 50, respectively. Two bands around 280 and 320$360 \mathrm{~nm}$ are seen in both absorption spectra. Although the latter absorption should be due to the silicon main chain, the former is due to the phenyl $\pi-\pi^{*}$ transition. It is noted that the UV absorption, FL, and FLA spectra of 49 and 50 reveals great differences.

For 49, the FL spectral profile with a fwhm of $30 \mathrm{~nm}$ is not the mirror image of the $340 \mathrm{~nm}$ absorption band and the photoexcitation wavelength depends strongly on the FLA around the $340 \mathrm{~nm}$ absorption band, ranging from 0.00 at $300 \mathrm{~nm}$ to 0.36 at $350 \mathrm{~nm}$. In contrast, for 50, the FL spectral profile with an fwhm of $15 \mathrm{~nm}$ is the mirror image of the $350 \mathrm{~nm}$ absorption band, although the FLA has a similar photoexcitation wavelength dependence around the $350 \mathrm{~nm}$ band, ranging from 0.00 at $300 \mathrm{~nm}$ to 0.31 at $360 \mathrm{~nm}$. These properties relate to the global main chain structure, since 


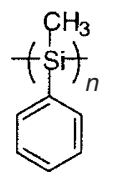

49

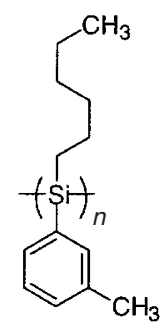

50

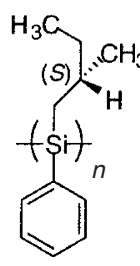

51

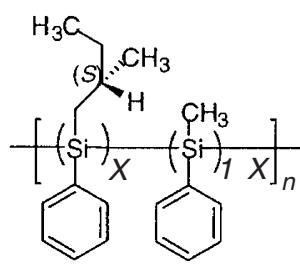

52

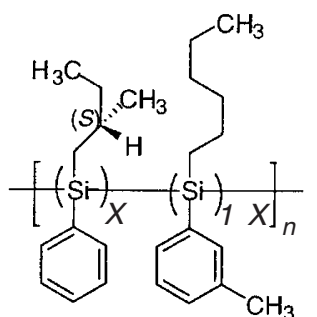

53

(not produced)

Chart 11. Chemical structures of poly[alkyl(alkylphenyl)silane] homopolymers $\mathbf{4 9}, \mathbf{5 0}$, and $\mathbf{5 1}$ (not formed), and copolymers $\mathbf{5 2}$ ( $\mathrm{x}=$ $0.05,0.10,0.25,0.30$, and 0.50$)$ and $\mathbf{5 3}(\mathrm{x}=0.05,0.10,0.20$, and 0.50$)$.
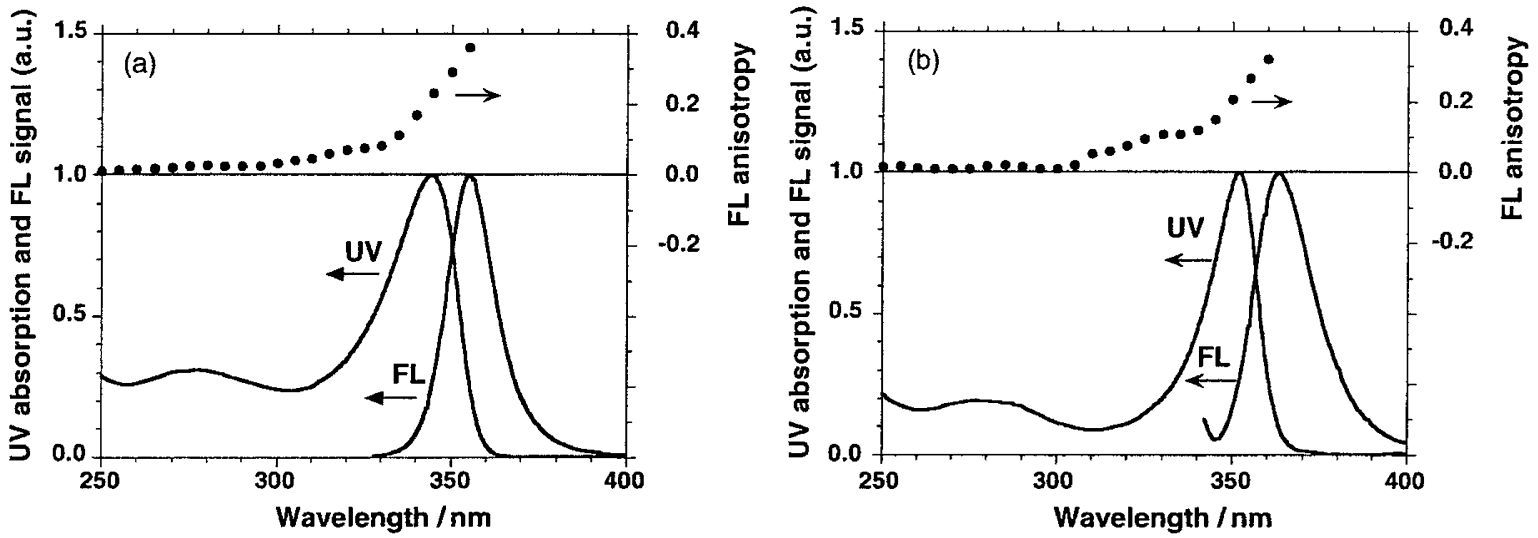

Figure 31. UV absorption, FL, and FLA spectra of poly[alkyl(alkylphenyl)silane] homopolymers (a) 49 and (b) $\mathbf{5 0}$ in THF at room temperature.

the polysilane absorption results from a collection of various chromophoric segments with different excitation energies. When segments with higher energy absorption are excited, the photoexcited electron-hole pair migrates into segments of lower energy from which emission then occurs. Consequently, the mirror-image relationship with narrower bandwidth between absorption and emission show that the main chain conformation of $\mathbf{5 0}$ has a more regular spatial conformation than that of 49. This may be consistent with the idea that 49 and 50 have a random-coil and stiff global conformational structures, respectively, in solution from the absorptivity-viscosity index-fwhm correlation. ${ }^{45}$

Molecular mechanics calculations support the idea that the most stable conformation of $\mathbf{4 9}$ and $\mathbf{5 0}$ is helical with equal proportions of $P$ - and $M$-motifs, resulting in optical inactivity. Figure 32a shows the potential energy of 49 oligomer model with twenty-one repeat units as a function of the $\mathrm{Si}-\mathrm{Si}-\mathrm{Si}-\mathrm{Si}$ dihedral angle. The respective $P$ - and $M$-helical conformations of $\mathbf{4 9}$ near $160^{\circ}$ and $200^{\circ}$ are more stable than the all-anti conformation of $180^{\circ}$ regardless of it- or st- configuration. The energy cost of helix reversal is estimated as $\sim 1 \mathrm{kcal}$ per repeat unit, suggesting a rapid twisting motion between $P$ - and $M$-motifs.
Figure $32 \mathrm{~b}$ shows the potential energy of oligo- $n$ hexyl(phenyl)silane, a model for $\mathbf{5 4}$ with thirty-one repeat units as a function of the dihedral angle instead of $\mathbf{5 0}$ for simplicity, since the conformations of $\mathbf{5 0}$ and $\mathbf{5 4}$ as can be seen from the similarity between the UV absorption and FL spectral profiles of $\mathbf{5 0}$ and $\mathbf{5 4}$. The respective $P$ - and $M$-helical conformations of it-54 near $170^{\circ}$ and $190^{\circ}$ are more stable than the all-anti conformation although the conformations of $s t \mathbf{- 5 4}$ are stable near $180^{\circ}$. If we accept from the plot that the potential energy of the $i t$-configuration is about $4 \mathrm{kcal}$ per repeat unit more stable than that of the $s t$ conformation at any dihedral angle, actual $\mathbf{5 0}$ is likely to contain mainly itsequences rather than $s t$-sequences.

These calculations and considerations led to the idea that $\mathbf{5 0}$ and $\mathbf{5 4}$ are likely to adopt helical structures as the most stable conformation, regardless of their optical activity.

\section{Helical Induction in Copolymers by the "Sergeants and} Soldiers" Principle

The UV absorption and CD spectra of copolymers $52(\mathrm{x}=0.25)$ and $53(\mathrm{x}=0.20)$ in $\mathrm{THF}$ at $23-25^{\circ} \mathrm{C}$ are shown in Figure 33a and 33b. The positive-sign CD spectral profile around $340 \mathrm{~nm}$ almost traces the corre- 

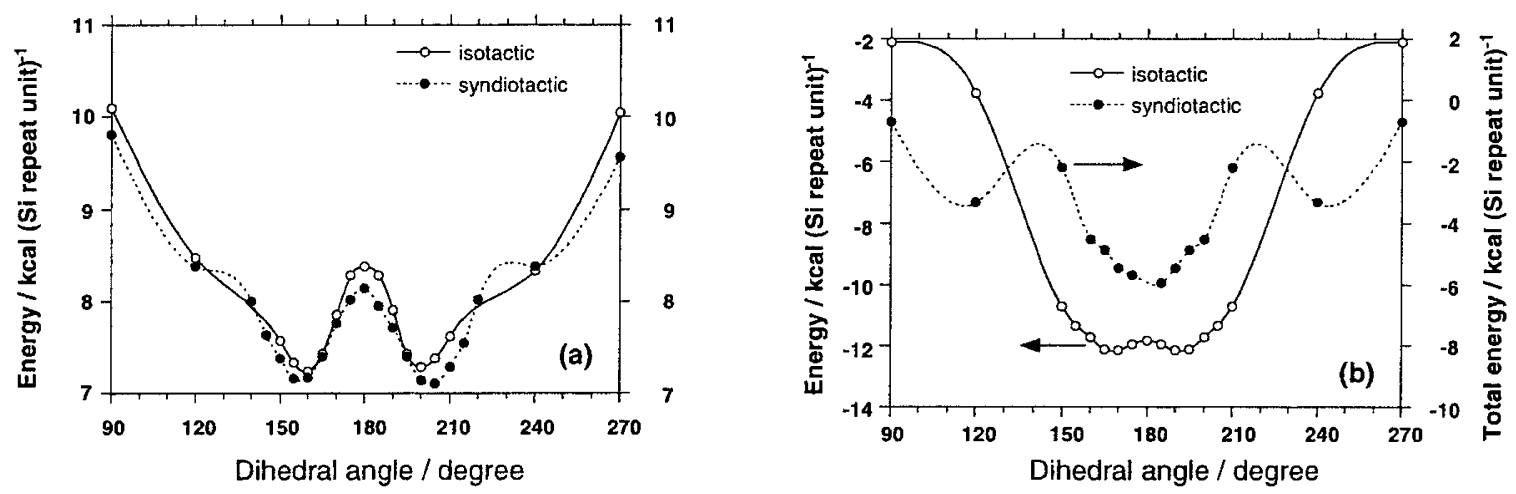

Figure 32. Potential energy per silicon repeat unit of (a) oligomethylphenylsilane (model for 49) with twenty-one silicon repeat units and (b) oligo- $n$-hexylphenylsilane (model for 54) with thirty-one silicon repeat units as a function of the Si-Si-Si-Si dihedral angle (MSI, Discover III, ver 3.00, a PCFF force-field).
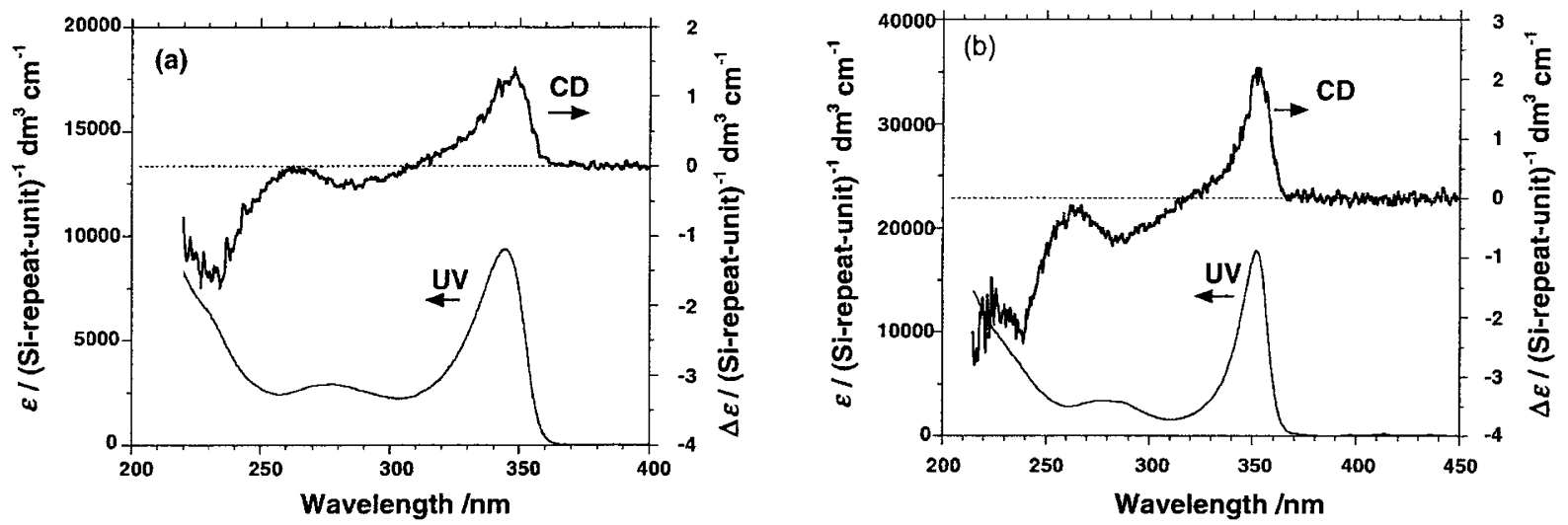

Figure 33. UV absorption and CD spectra of (a) copolymers $52(x=0.25)$ and $(b)$ copolymers $\mathbf{5 3}(\mathrm{x}=0.20)$ in $\mathrm{THF}$ at $23-25^{\circ} \mathrm{C}$.

sponding $340 \mathrm{~nm}$ UV absorption for both copolymers. This is consistent with helical conformations for $\mathbf{4 9}$ and 50, since, if 49 and 50 have an achiral, all-anti conformation, a zero or far smaller CD signal should be observed. If $\mathbf{5 2}$ and $\mathbf{5 3}$ adopt partly all-anti segments, which cannot be responsible for a CD signal in the main chain, the CD band will not completely match the apparent UV band. All UV-CD spectral profiles for all the copolymers with different proportions of chiral side moieties, $\mathbf{5 2}$ and 53, were almost identical except for the magnitude of the $\mathrm{CD}$ band. This therefore led to the idea that the local conformations of optically active 52 and 53 should be helical with a preferential screwsense. It is considered from this extrapolation, that even optically inactive 49 and 50 may have a latent helical structure, ${ }^{50}$ but without a preferential screw-sense.

To discuss the preferential screw sense capability qualitatively in a series of $\mathbf{5 2}$ and $\mathbf{5 3}$ copolymers with chiral-achiral side chains, the $g_{\text {abs }}$ values are more convenient for quantatively discussing the conformational changes as a function of the chiral mole composition than the $\Delta \varepsilon$ value, since it has already been established in numbers of optically active poly(dialkylsilane) homopolymers and copolymers, that the $g_{\text {abs }}$ value is very sensitive to changes in $P$ - and $M$-helix populations ${ }^{31}$

Figure $34 \mathrm{a}$ and $34 \mathrm{~b}$ show the values of the values of $g_{\text {abs }}$ at CD-UV peak absorption wavelengths in a series of $\mathbf{5 2}$ and $\mathbf{5 3}$ as a function of the chiral mole composition.

Evidently, although the $g_{\text {abs }}$ values for $\mathbf{5 2}$ and $\mathbf{5 3}$ increases nonlinearly as the mole composition of the chiral units increases, there is a certain difference in the relation between the $g_{\text {abs }}$ values and the chiral composition. ${ }^{32 \mathrm{f}}$ The $g_{\text {abs }}$ values of $\mathbf{5 2}$ increases almost linearly as the chiral composition increases from zero to $30 \mathrm{~mol} \%$, and eventually converges at a chiral composition of $30 \mathrm{~mol} \%$. In contrast, the $g_{\text {abs }}$ values of 53 increases nonlinearly with convex curvature as the chiral composition increases from zero to $50 \mathrm{~mol} \%$. Although poly[(S)-2-methylbutyl(phenyl)silane] homopolymer (51) was not produced, probably because of the great steric effect of the $(S)$-2-methylbutyl moieties attached to the main chain, we consider these differences to originate from the chain stiffness and/or persistence length differences between 49 and $\mathbf{5 0}$.

The introduction of $(S)$-2-methylbutyl substituents, however, does not cause any great change in their global or local main chain conformations including di- 

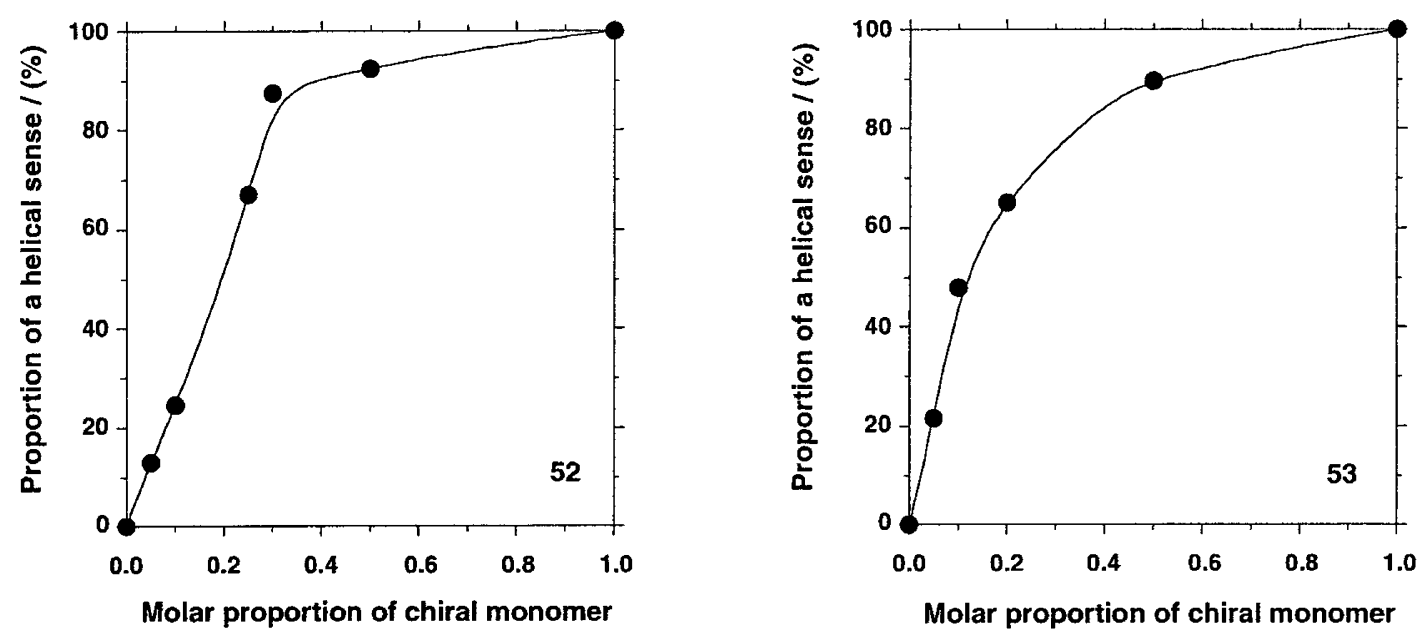

Figure 34. Proportion of preferential helical screw sense as a function of mole fraction of chiral substituents in (left) copolymers $\mathbf{5 2}$ and (right) copolymers 53.

hedral angle, as discussed above. The population of $P$ - and $M$-motifs, therefore, changes nonlinearly as regards the chiral unit composition. We may call these relations between $g_{\mathrm{abs}}$ values and the chiral composition a positive cooperative effect ("sergeant-good soldiers") for preferential screw-sense helix induction, as already established in poly(alkylisocyanate) copolymers bearing chiral and achiral side chains. ${ }^{9,18 b}$

In a series of rigid rod-like helix poly(dialkylsilane)s with a single-screw-sense, the maximum $g_{\text {abs }}$ values $\left(g_{\max }\right)$ reaches $\sim 2.0 \times 10^{-4}$ at the UV peak absorption wavelength, ${ }^{30 f, 30 \mathrm{k}}$ while that of $\mathbf{4 9}(\mathrm{x}=0.30)$ is slightly lower, $\sim 1.7 \times 10^{-4}$. If the value of $g_{\max }$ of poly[alkyl(aryl)silane] derivatives (52 and 53) with a $100 \%$ pure $P$ - or $M$-helix is assumed to $\sim 1.8 \times 10^{-4}$ by extrapolation, ${ }^{32 \mathrm{f}}$ the presence of about $30 \%$ chiral units can effectively induce an approximately $95 \%$ positive sign $\mathrm{CD}$ helix with 5\% negative sign CD helix resulting in a $90 \%$ enantiomeric excess of the positive sign CD pseudoenantiomer of 49.

Two series of $\mathbf{5 2}$ copolymers with a coiled main chain structure and $\mathbf{5 3}$ copolymers with a stiff main chain conformation were described. It was concluded by means of UV and CD spectra, as well as molecular mechanics calculations, that both optically inactive 49 and 50 should adopt helical conformations with an equal quantity of $P$ - and $M$-screw-senses. A marked "positive" cooperative induction phenomenon of preferential screw-sense was found in $\mathbf{5 2}$ and $\mathbf{5 3}$ copolymers. However, there is a marked difference in the helical cooperativity between $\mathbf{5 2}$ and $\mathbf{5 3}$, probably because of the differences in their global and local conformations. This difference may be connected to the persistence length of the helical conformation limited by helix reversals which allow change of screw sense as seen originally in the work of the Italian school on isotactic vinyl polymers $^{15 \mathrm{c}}$ and later in the polyisocyanates. ${ }^{9,18 \mathrm{~b}}$

\section{OPTICALLY ACTIVE POLY(DIARYLSILANE)S}

\section{$C D$ and UV Spectra of Homopolymers}

Amongst the polysilanes, those featuring two aryl ring side chains exhibit the longest wavelength UV absorption, around $400 \mathrm{~nm},{ }^{47}$ although the actual value depends on the aryl ring substituent(s). To afford reasonable solubility, alkyl chains of four or more longer carbon atoms should be attached to the aryl rings. Theoretical calculations indicate that both opening of the silicon main chain dihedral angles and interaction between the phenyl ring $\pi$-orbitals and the silicon main chain result in a decreased optical band gap, causing the absorption maximum to shift to longer wavelengths ${ }^{36,51}$ The local conformation of the poly(diarylsilane)s was long considered to consist of a fully extended, all-anti silicon main chain from both theoretical ${ }^{36,76 \mathrm{~d}}$ and experimental ${ }^{76 \mathrm{a}-76 \mathrm{c}}$ perspectives. However, X-ray analysis of diphenylsilane oligomer single crystals ${ }^{77}$ revealed helical main chains with dihedral angles around $150-160^{\circ}$. Our molecular mechanics calculations ${ }^{33 c}$ on thirty-mer chains were consistent with this, affording non-minimum potential energies for all-anti structures. To further test experimentally whether poly(diarlysilane)s adopt helical or all-anti main chain conformations, a series of homopolymers ${ }^{33 a}$ and copolymers featuring enantiopure chiral and achiral alkyl substituents on the aryl rings were systematically investigated. ${ }^{33 b, 33 c}$ Firstly, facile control of screw sense in poly(diaryl)silane homopolymers is presented, and secondly copolymers exhibiting cooperative helix induction and helix inversion phenomena are described. ${ }^{33 \mathrm{~b}}$

The chemical structures of poly(diarylsilane) ho- 


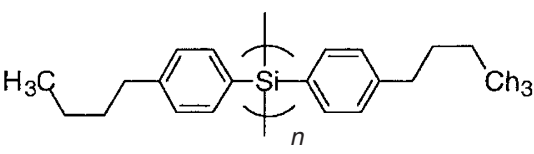

54
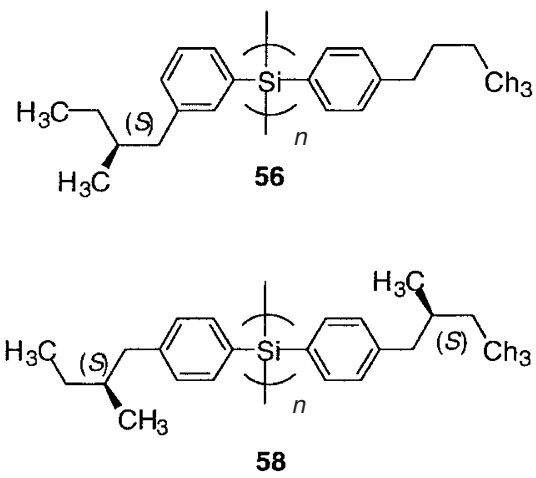

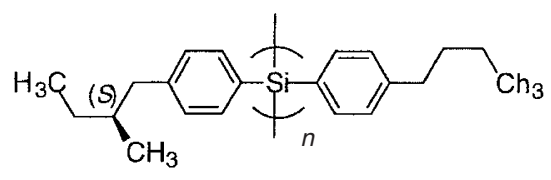

55

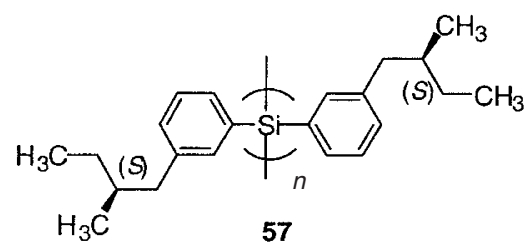

Chart 12. Chemical structures of poly(diarylsilane) derivatives, 54-58.
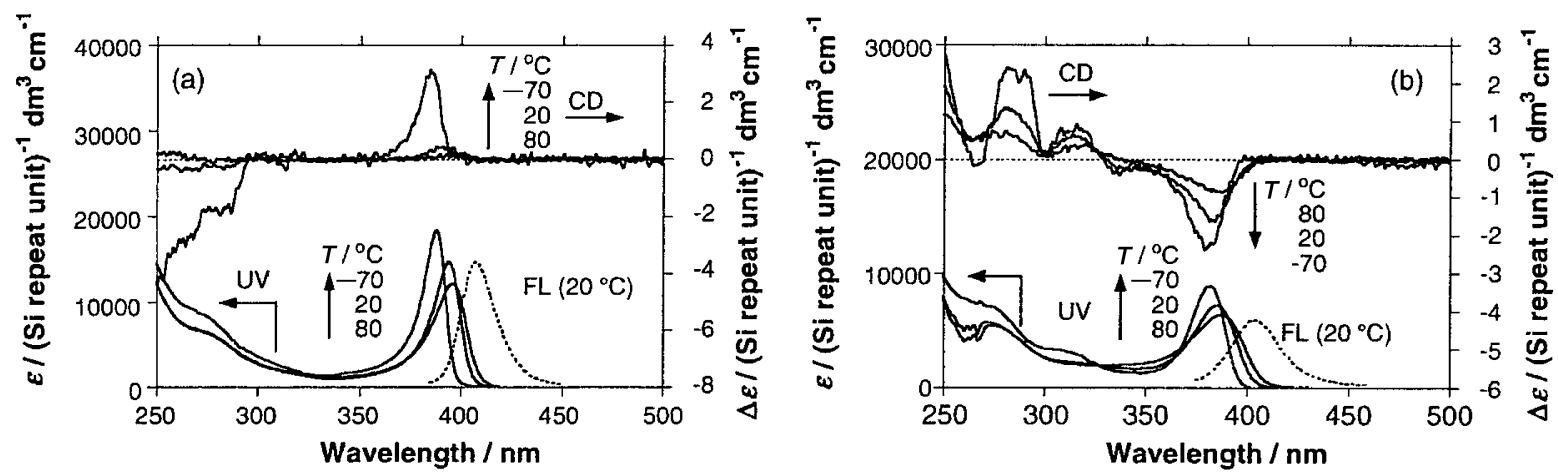

Figure 35. Variable temperature CD, UV, and fluorescence spectra of $\mathbf{5 5}$ and $\mathbf{5 7}$ in isooctane.

mopolymers 54-58 are shown in Chart 12. Since polysilane main chains are both fluorophoric and chromophoric due to delocalisation of the silicon $\sigma-\sigma^{*}$ transition, the CD, UV-Vis and fluorescence spectroscopic features are particularly useful to characterize the conformational structures, as noted above. The magnitude of the $g_{\text {abs }}$ value is again of use.

Polysilanes 54-58 show relatively narrow UV absorptions with fwhm of $c a .16 \mathrm{~nm}$ around $396 \mathrm{~nm}$ and mirror image fluorescence emission spectra with small Stokes shifts of $\sim 13 \mathrm{~nm} .{ }^{33}$ Additionally, fluorescence anisotropies in the range $0.20-0.38$ indicate regular, stiff polymer molecules with long segment lengths in solution. ${ }^{30 \mathrm{~d}}$

Figure $35 \mathrm{a}$ and $35 \mathrm{~b}$ show the variable temperature $\mathrm{CD}$ and $\mathrm{UV}$-visible spectra between -70 and $80^{\circ} \mathrm{C}$, and FL spectra at $20^{\circ} \mathrm{C}$ for $\mathbf{5 5}$ with one para- $(S)$ 2-methylbutyl group per repeat unit and $\mathbf{5 7}$ with two meta-(S)-2-methylbutyl groups per repeat unit in isooctane. The signs of the CD spectra for these polymers (positive and negative, respectively) indicate that, despite containing the same $(S)$ enantiomer group, they show opposite helical chirality. Analysis of 56, with one meta-(S)-2-methylbutyl group per repeat also revealed a positive sign $\mathrm{CD}$ signal, while $\mathbf{5 8}$ with two para-(S)-2-methylbutyl groups also showed negative $\mathrm{CD}$, coincident with the corresponding UV-Vis absorption spectra.

From Figure 35a, the adoption of this helical structure results from a certain relationship between the alkyl substituents on neighboring phenyl rings. While the UV spectra of the poly(diarylsilane)s are similar, the CD spectra depend strongly on the polymer composition. It appears that the helical screw sense depends on the number of chiral centers per repeat unit - one producing a positive $\mathrm{CD}$ helix and two producing a negative $\mathrm{CD}$ helix. For 55, the Cotton effect is greatest at $-70^{\circ} \mathrm{C}$, though as the temperature is raised the effect decreases to around zero at $80^{\circ} \mathrm{C}$. This indicates that the conformation of the helical silicon chain is dynamically twisting between the two screw senses. At low temperature this process is slow and the preferential screw sense emerges, while at high temperature, the process is fast, effectively equilibrating the popu- 
lations of the two screw senses. The corollary of the reduction in Cotton effect intensity at high temperature is an increase in $\Delta \varepsilon$ with decrease in temperature. This effect has also been observed by other workers. Green et al. demonstrated that the optical activity of poly(alkylisocyanate)s increases with decreasing temperature due to a diminishing number of helix reversals, allowing greater cooperation of main chain units. ${ }^{18 \mathrm{~b}}$

Since the CD spectrum for $\mathbf{5 6}$ exhibits a positive Cotton effect at the lowest energy UV transition, the main chain should adopt the same preferential screw sense as that for $\mathbf{5 5}$, although the small value of $g_{a b s}$ $\left(=+0.34 \times 10^{-4}\right)$ suggests weaker helical screw sense preference such as a partial cancellation of oppositely signed CD bands of major and opposite minor screw sense pseudo-enantiomeric helical segments. ${ }^{33 \mathrm{~b}}$ The weaker screw sense preference may be a result of the greater ability of $\mathbf{5 4}$ to mitigate steric congestion by phenyl ring rotation to minimize interaction between the meta chiral alkyl groups on nearest and next nearest silicon atoms. ${ }^{32 \mathrm{c}}$

In contrast, the CD spectra of $\mathbf{5 7}$ and $\mathbf{5 8}$ exhibit negative Cotton effects, as exemplified in Figure $35 \mathrm{~b}$ for 57. This implies a helical conformation, but of the opposite preferential screw sense to $\mathbf{5 5}$ and $\mathbf{5 6}$, even though the enantiopure chiral moiety in all cases is the (S)-2-methylbutyl group. Similarly, the Cotton effect is of greatest absolute magnitude with negative sign at $-70^{\circ} \mathrm{C}$ and almost zero at $80^{\circ} \mathrm{C}$.

The finding that poly(diarylsilane)s exhibiting either positive or negative $\mathrm{CD}$ signals-i.e., with opposing screw senses-may be obtained using only $(S)$-chiral substituents, is significant in synthetic polymer chemsitry in that to obtain helical homopolymers with opposing screw senses, it may be not necessary to use both enantiomeric forms of the chiral group. The cheaper enantiomer may therefore be employed in any industrial applications to obtain both pseudoenantiomeric helical polymer conformations.

It may be possible that the helicity in poly(diarylsilane)s is a result of induction by the chiral substitution interaction, and that polymers with achiral substituents actually have all-anti conformations. While this possibility cannot be directly ruled out, comparison of the spectroscopic data of polymers with chiral substituents and those with achiral substituents. e.g., $\mathbf{5 4}$ and 58, respectively, indicates similar main chain dihedral angles, since the UV absorption maxima are so similar. Both polymers should therefore be helical, with the difference being that in the case of 54 the overall numbers of $P$ - and $M$-turns are equal, whereas for $\mathbf{5 8}$, one of the screw senses predominates, resulting in net helicity and optical activity.

\section{Molecular Mechanics Calculations}

For poly(diarylsilane)s with achiral and chiral substituents, molecular mechanics calculations of model oligomers afforded almost symmetrical tetra-well potential energy curves with non-minimum $180^{\circ}$ values in both $\mathrm{H}-\left(\mathrm{Ph}_{2} \mathrm{Si}\right)_{30}-\mathrm{H}$ (model of unsubstituted diphenylpolysilane, 59) and $\mathrm{H}-\left[(p-n-\mathrm{BuPh})_{2} \mathrm{Si}\right]_{30}-\mathrm{H}$ (model for 54), and provide asymmetrical tri-well potential energy curves with non-minimum $180^{\circ}$ values in (c) $\mathrm{H}-\left(\mathrm{Ar}_{2}{ }_{2} \mathrm{Si}_{30}-\mathrm{H}\right.$ (model for 58), (d) $s t-\mathrm{H}-$ $(\mathrm{ArAr} * \mathrm{Si})_{30}-\mathrm{H}$, and (e) $i t-\mathrm{H}-(\mathrm{ArAr} * \mathrm{Si})_{30}-\mathrm{H}$ models for 55) (where $\mathrm{Ar}=p$ - $n$-butylphenyl, and $\mathrm{Ar}^{*}=p-(S)-$ 2-methylbutylphenyl), respectively, as shown in Figure 36a-36e.

These calculations predict helical main chain conformations of poly(diarylsilane)s, regardless of the presence of chiral or achiral substituents. For $\mathbf{5 4}$ and even 59, equal proportions of $P$ - and $M$-turns should exist, resulting in overall optical inactivity of the internal racemate. In contrast to previous reports, which suggested an all-anti conformation for perphenylated oligosilanes, ${ }^{77}$ the present calculations indicate a latent helical conformation for $\mathbf{5 4}$, in agreement with our experimental observations. However, calculation for a polysilane with small substituents, i.e., $\mathrm{Cl}-\left(\mathrm{Cl}_{2} \mathrm{Si}\right)_{30^{-}}$ $\mathrm{Cl}$ did suggest an all-anti conformation ${ }^{69}$ with a potential energy minimum at $180^{\circ}$ in agreement with the experimental single crystal X-ray structure determination, though with all other, bulkier substituents (aryl and aliphatic), helical structures are predicted. ${ }^{32 \mathrm{c}}$

Calculations of poly(diarylsilane) models $\mathbf{5 5}$ and $\mathbf{5 8}$, however, suggest that both $P$ - and $M$ - screw senses should coexist, but that the lower energy $M$-turns should be in greater abundance, resulting in an excess of these and consequent optical activity, corroborating our experimental conclusions from CD studies of helicity. In contrast to experiment, though, the calculations did not indicate opposite screw sense preferences for models with one and two enantiopure chiral groups per monomer unit.

\section{Temperature and Composition Effects of Helical Coop- erativity in Copolymers}

Of great interest concerning copolymers are cooperativity effects such as helical amplification and screw sense inversion, as exemplified in poly(dialkylsilane)s and poly(alkylarylsilane)s. It is now established for the helical polyisocyanates ${ }^{9,18 e, 18 p, 18 t}$ that side chain interactions can result in significant preferential screw sense induction within long stiff segments and that chiral monomer units can exert stereochemical control over the helicity of adjacent achiral monomer units by the "sergeants and soldiers effect". It was of interest to in- 

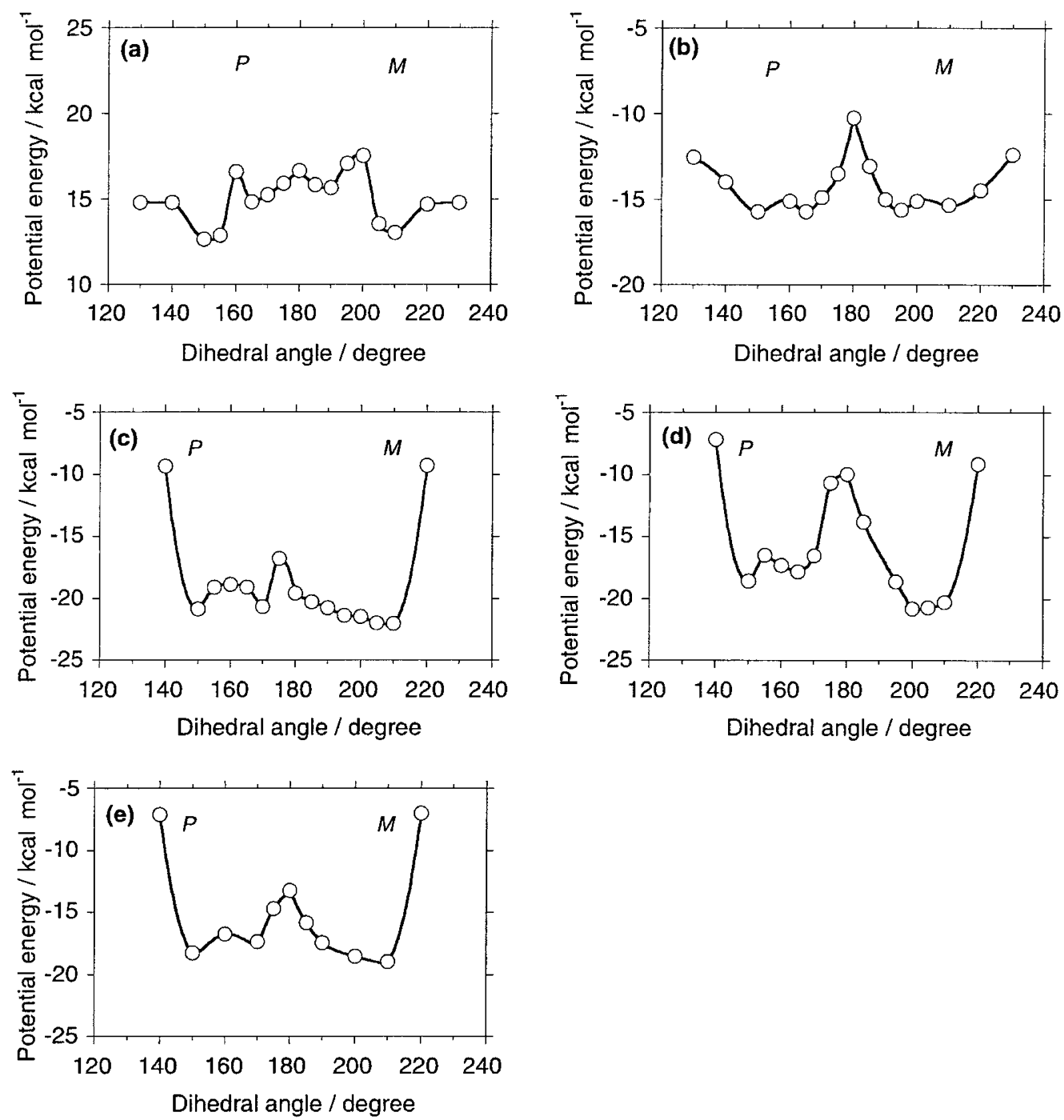

Figure 36. Potential energy per repeat unit as a function of main chain dihedral angle for model poly(diarylsilane)s with achiral substituents, (a) $\mathrm{H}-\left(\mathrm{Ph}_{2} \mathrm{Si}\right)_{30}-\mathrm{H}$ (59), (b) $\mathrm{H}-\left[(p-n-\mathrm{BuPh})_{2} \mathrm{Si}\right]_{30}-\mathrm{H}$ (54), and for model poly(diarylsilane)s with chiral substituents, (c) $\mathrm{H}-\left(\mathrm{Ar}_{2}{ }_{2} \mathrm{Si}\right)_{30}-\mathrm{H}(\mathbf{5 8})$, (d) $s t-\mathrm{H}-(\mathrm{ArAr} * \mathrm{Si})_{30}-\mathrm{H}$, and (e) $i t-\mathrm{H}-(\mathrm{ArAr} * \mathrm{Si})_{30}-\mathrm{H}(\mathbf{5 5})$ (where $\mathrm{Ar}=p$ - $n$-butylphenyl, and $\mathrm{Ar} *=p-(S)-2-$ methylbutylphenyl). Calculations performed using MSI, Discover 3, ver. 4.00, pcff force field.

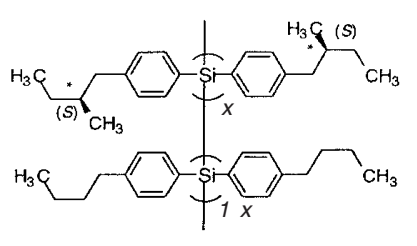

60: $x=0.2$

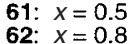

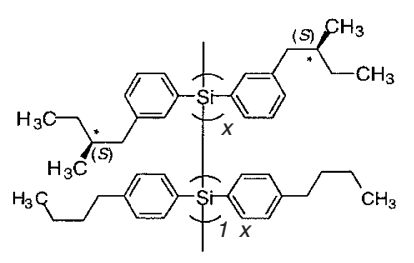

63: $x=0.2$

64: $x=0.5$

Chart 13. Chemical structure of optically active poly(diarylsilane) copolymers 60-65.

vestigate whether such an effect could be observed for the poly(diarylsilane)s, since in any device application, considerable saving in costs may be achievable if preferential screw sense helical polymers could be obtained from largely achiral materials.

Similarly to the homopolymers, spectroscopic studies of the copolymers were carried out in dilute solution $\left(1 \times 10^{-4} \mathrm{M}\right.$ in toluene, isooctane or THF) between -70 and $80^{\circ} \mathrm{C}$. As is evident in Figure 37, the CD spec- trum of $\mathbf{6 1}$ exhibits a positively signed Cotton effect around $390 \mathrm{~nm}$, almost coincident with the lowest energy main chain $\sigma-\sigma^{*}$ transition in the $20^{\circ} \mathrm{C}$ UV spectrum at $394 \mathrm{~nm}$. The CD spectra of $\mathbf{6 0}$ and $\mathbf{6 2}$ show similar features to that of $\mathbf{6 1}$, and the similarity of these data with those for the homopolymers indicates that the copolymers are therefore expected to adopt helical conformations.

To investigate helical cooperativity in these copoly- 


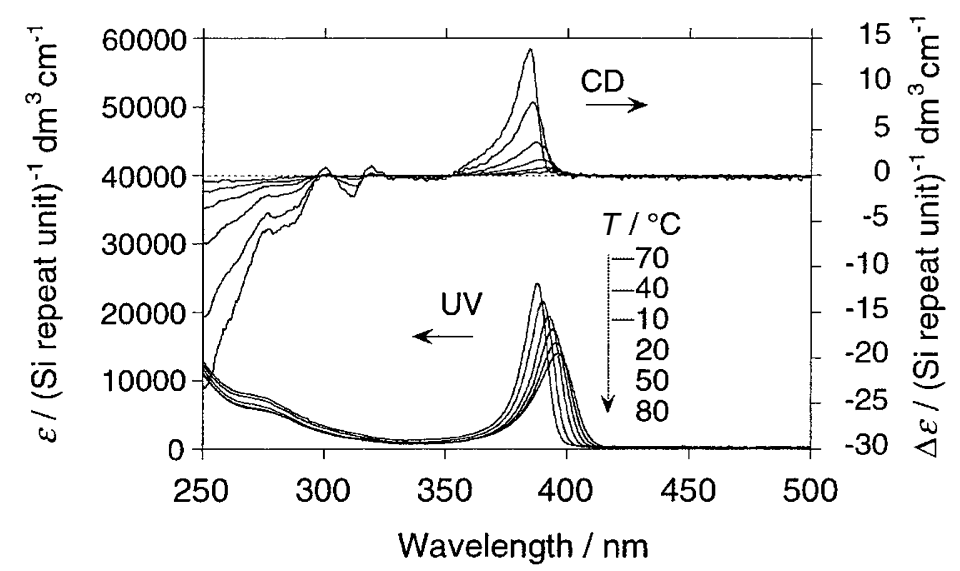

Figure 37. Variable temperature CD and UV spectra of $\mathbf{6 1}$ in isooctane.

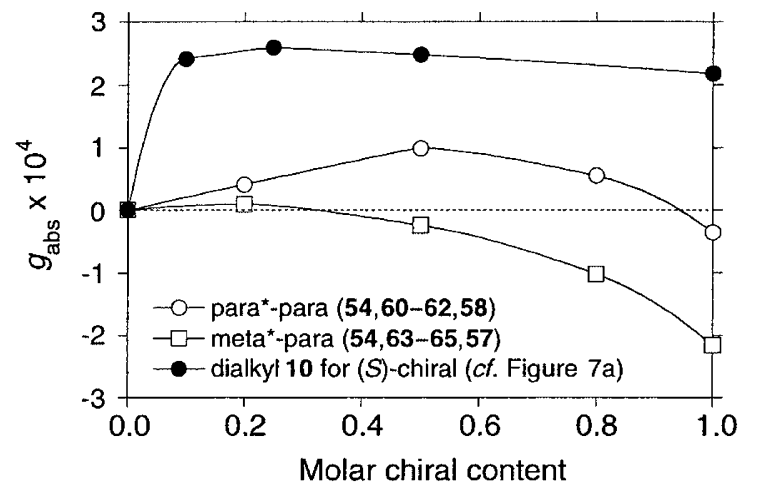

Figure 38. Dissymmetry ratio, $g_{\text {abs }}$ as a function of chiral content for copolymers $(\mathbf{5 4}, \mathbf{5 7}, \mathbf{5 8}, \mathbf{6 0}-\mathbf{6 5})$ in toluene at $20^{\circ} \mathrm{C}$.

mer systems, the Kuhn dissymmetry ratios, $g_{\text {abs }}$, were evaluated. The value of $g_{\text {abs }}$ is displayed as a function of chiral content for $54,57,58$, and $60-65$ at $20^{\circ} \mathrm{C}$ in Figure 38. It is evident that the absolute magnitudes of the $g_{\text {abs }}$ value for the para-copolymers 60-62 exceed those for enantiopure homopolymer 58. Since the UV, FL, FLE, and FLA spectroscopic data are similar for all the polymers, it is reasonable to assume that polymer main chain dihedral angles are similar, such that an increase in $g_{\text {abs }}$ should imply greater helical screw sense induction. The larger $g_{\text {abs }}$ values for copolymers 60-62 may therefore indicate that the cooperative effect in the para-phenyl-substituted copolymers $\mathbf{5 8}$ is such as to induce helical main chain conformations with greater screw sense selectivity than the enantiopure-substituted poly(diarylsilane) homopolymer, in which there is assumed to be a minor presence of segments of the less favored, opposite, chiral sense, resulting in a lower dissymmetry ratio.

Among 60-62, 61 with a ratio of $(S)$-chiral $/ n$ butyl $=0.5 / 0.5$ shows the greatest absolute value of $g_{\text {abs. }}$. At higher chiral content than this, the value of $g_{\text {abs }}$ decreases again, inverting to negative for the bis-chiral homopolymer $\mathbf{5 8},{ }^{30 a, 30 c}$ indicating a change of preferential screw sense. This unusual cooperativity contrasts with that observed in other optically active copolymers such as poly(arylisocyanate)s, ${ }^{18 \mathrm{~m}, 18 \mathrm{n}}$ poly(alkylisocyanate)s, ${ }^{18 \mathrm{~b}}$ and poly(dialkylsilane)s. ${ }^{30 a-30 c, 30 g}$ In these latter two cases, the screw sense is determined by a small minority of enantiopure chiral repeat units and the dissymmetric ratio is constant or only slightly increases at greater chiral content levels. ${ }^{30 \mathrm{~g}}$ The CD and UV spectra of all three polymers 60-62 exhibit temperature dependence as is evident for $\mathbf{6 1}$ in Figure 37 and presented graphically for all the copolymers in Figure 39a and 39b (which show plots of UV and CD $\lambda_{\max }$ intensities vs. temperature, respectively), the UV $\lambda_{\max }$ shifting to slightly longer values at higher temperatures with generally a concomitant reduction in intensity and the $\mathrm{CD}$ intensity dropping in most cases to nearly zero value at about $80^{\circ} \mathrm{C}$. This is consistent with the loss of screw sense preference due to the thermal energy and consequent partial cancellation of oppositely signed CD bands.

In contrast, however, the $\mathrm{CD}$ spectrum of 64 , containing $50 \%$ bis- $m-(S)-2$-methylbutylphenyl-substituted monomer units, exhibits very weak negative $\mathrm{CD}$ bands (dissymmetry ratio at $-10^{\circ} \mathrm{C}, g_{\text {abs }}=-0.23 \times 10^{-4}$ ), lower by a factor of $4-8$ than those of 60-62, indicating that there is only a weak cooperative effect in the meta case, the main chain adopting the opposite screw sense to the para-substituted cases, presumably resulting from the better alleviation of steric hindrance afforded. Two possible, non-exclusive, reasons can be considered to account for the lower dissymmetry ratio: (i) The polymer main chain dihedral angles are very close to $180^{\circ}$, resulting in a looser helix. (ii) Although the main chain is helical, there is less dominant screw sense selectivity, thus giving rise to weaker Cotton effects through partial cancellation of oppositely signed CD bands. ${ }^{33 \mathrm{c}}$ This may result from the greater ability of 55 to mitigate steric congestion by phenyl ring rotation, minimizing interaction between the meta chiral alkyl 

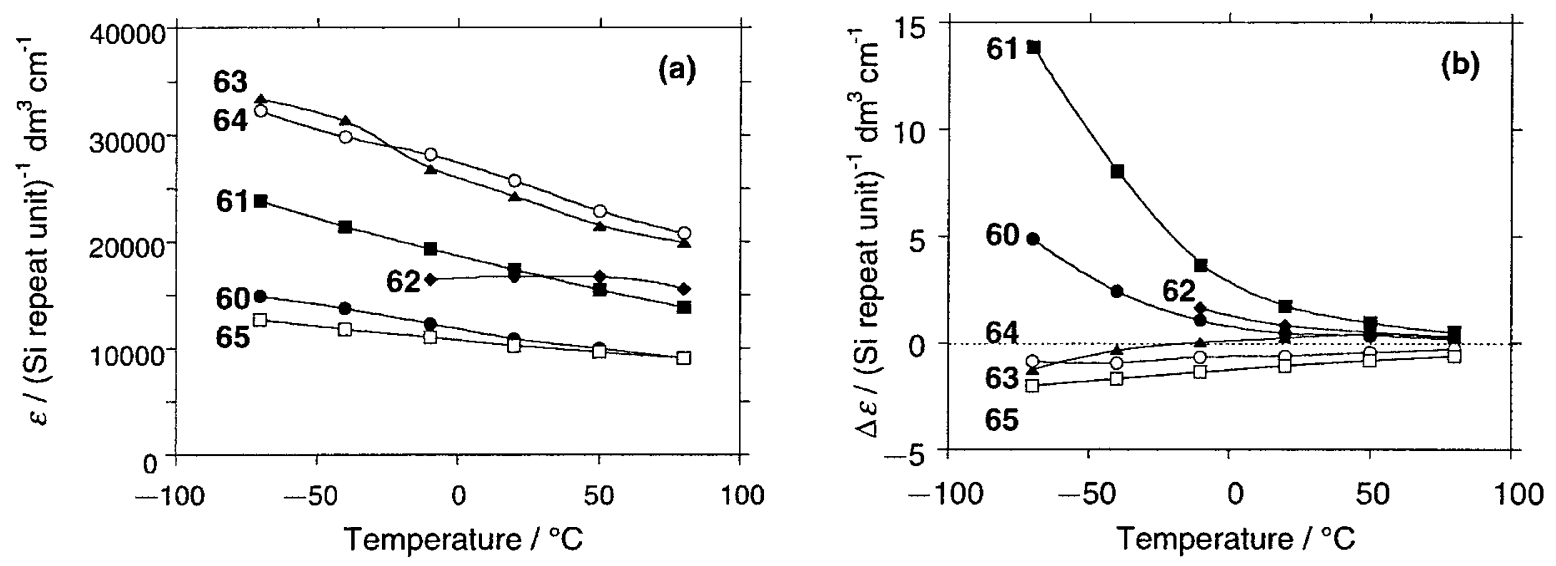

Figure 39. Plots of (a) UV and (b) CD $\lambda_{\max }$ intensities of $60-65$ s. temperature in toluene.
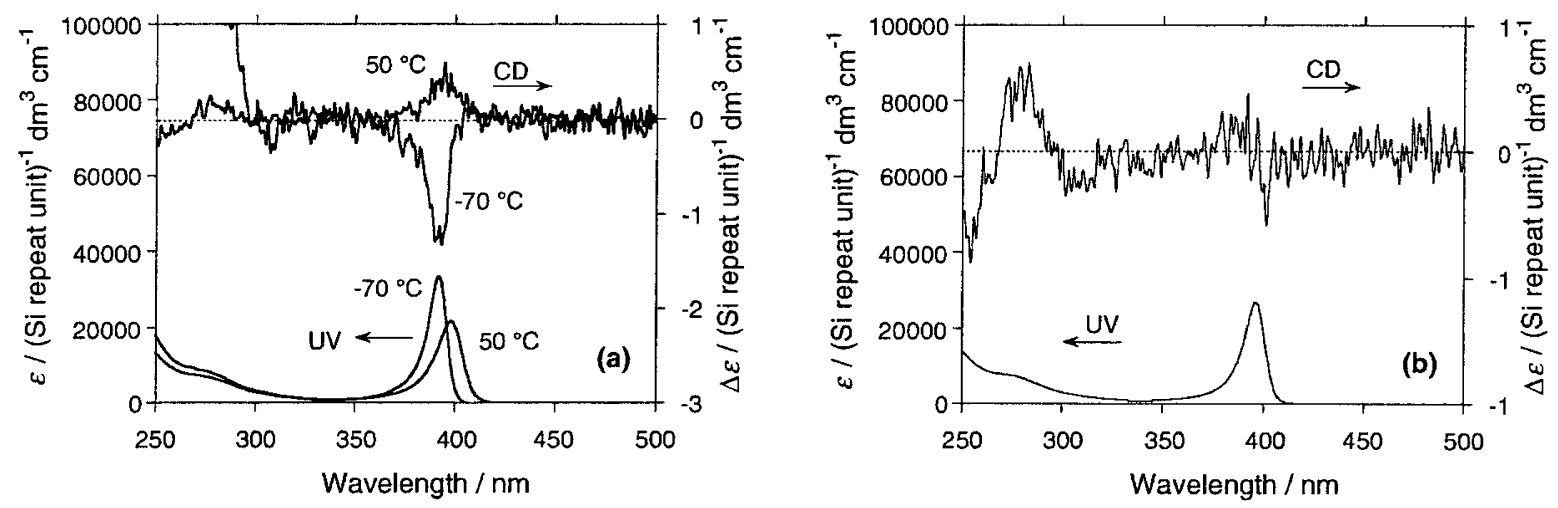

Figure 40. $\mathrm{CD}$ and $\mathrm{UV}$ spectra for helix-helix transition copolymer, $\mathbf{6 3}$, in isooctane (a) at -70 and $50^{\circ} \mathrm{C}$ and (b) at $-10^{\circ} \mathrm{C}$.

groups and $p$ - $n$-butyl or $m$-(S)-2-methylbutyl groups on neighboring silicon atoms. The room temperature spectroscopic data for all the homo- and copolymers are similar, though 64 exhibits a longer UV absorption maximum (indicative of more open main chain dihedral angles) than 63 and $65 .{ }^{33 \mathrm{c}}$ Although it is not possible at present to definitively state the origin of the weaker Cotton effects observed for $\mathbf{6 4}$, we suggest that both factors outlined above are responsible, viz., 64 has a loose helical structure (near to all anti) in which $P$ and $M$ sense helical turns coexist but in unequal proportions. For copolymer 65, similar spectral features are observed to 64, though with increasing negative dissymmetry ratios in the meta-phenyl-substituted series. Among all the poly(diarylsilane)s studied, it is homopolymer $\mathbf{5 7}$ which evidences the highest dissymmetry ratio.

\section{Thermo-Driven Helix-Helix Transition}

In the case of $\mathbf{6 3}$, containing $20 \% m-(S)-2-$ methylbutylphenylsilane units, the $\mathrm{CD}$ spectrum shows a negative Cotton effect at $-70^{\circ} \mathrm{C}$, as can be seen in Figure $40 \mathrm{a}, 40 \mathrm{~b}$, whereas at $50^{\circ} \mathrm{C}$, the effect is positive. At $-10^{\circ} \mathrm{C}$ the signal is approximately zero. $33 \mathrm{~b}$ This observations indicate a change in the main chain from one prevailing helical screw sense to the other, a rare example of a temperature-driven helical inversion of a polymer in dilute solution. There are a few other reports of such solution state inversions, these concern, e.g., certain poly $(\alpha$-amino acid ester $) \mathrm{s}^{53,54}$ a poly(aryl isocyanate), ${ }^{18 f, 18 p}$ poly(dialkylsilane)s, ${ }^{31}$

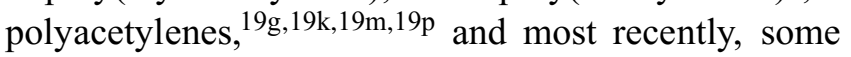
polyisocyanate copolymers. ${ }^{18 t}$

The small $g_{\text {abs }}$ value for 63 at $20^{\circ} \mathrm{C}$ may imply proximity to the transition temperature. However, the absolute magnitude of $g_{\text {abs }}$ for 63 at $-70^{\circ} \mathrm{C}\left(-0.38 \times 10^{-4}\right)$ is greater than that for $64\left(-0.27 \times 10^{-4}\right)$, but less than that for $60\left(-1.57 \times 10^{-4}\right)$. By comparison, the $g_{\text {abs }}$ value for the related bis- $m$-chiral homopolymer $\mathbf{5 7}$ is $-2.00 \times 10^{-4}$ at $20^{\circ} \mathrm{C} .{ }^{30 a}$ The lower dissymmetric ratios for the bis- $m$-chiral copolymers 63-65, therefore, are most probably the consequence of the coexistence of both helical screw senses in their main chains at all temperatures.

Consistent with the above suggestion, the $\mathrm{CD}$ spectrum of 63 at $-10^{\circ} \mathrm{C}$ actually has a small negative component at $\sim 401 \mathrm{~nm}$ in addition to the small positive peak at $390 \mathrm{~nm}$, as shown in Figure 40b. These two bands are ascribed to the two different screw senses, which should be characterized by different screw pitches. ${ }^{33 \mathrm{~b}}$ It 

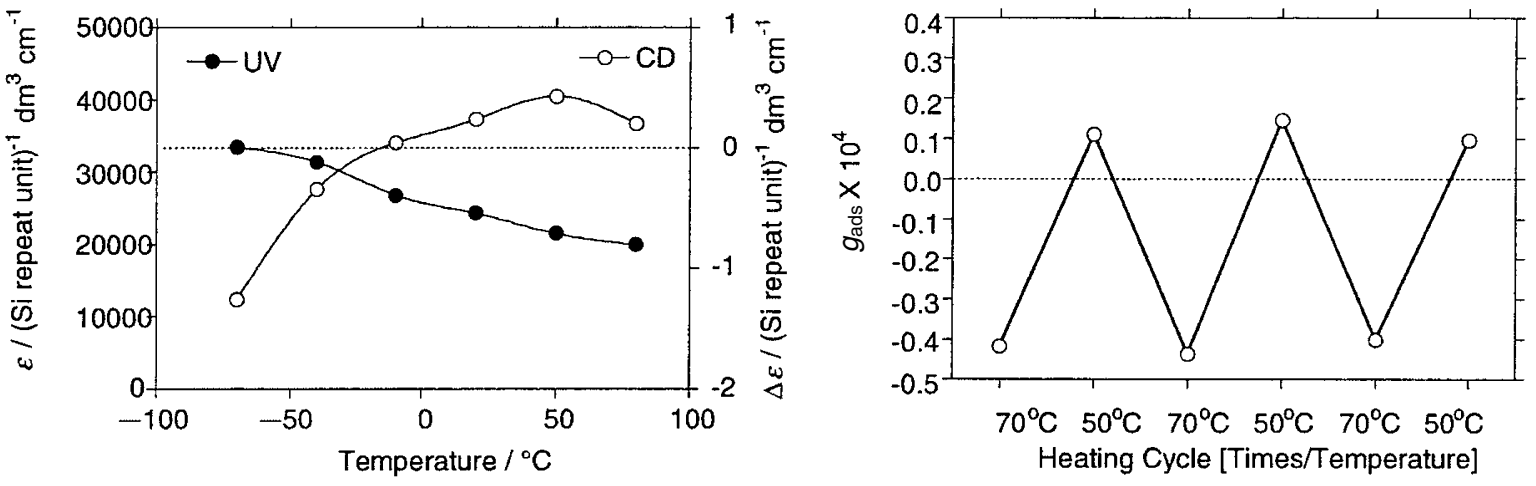

Figure 41. (a) CD and UV intensities $v s$. temperature for $\mathbf{6 3}$, showing a helix-helix transition in toluene. (b) Temperature cycling experiment for $\mathbf{6 3}$ in toluene.

is not possible, however, to associate a particular screw sense with the sign of the Cotton effect. The maximum magnitude of the positive Cotton effect for $\mathbf{6 3}$ occurs around $50^{\circ} \mathrm{C}$, but it is less than the maximum negative magnitude, as may be seen from the plot of $\mathrm{CD}$ intensity $\Delta \varepsilon v s . T$ in Figure $37 \mathrm{~b}$.

Above $50^{\circ} \mathrm{C}$, the $\mathrm{CD}$ signal intensity decreases again, a feature in common with the other poly(diarylsilane)s, ${ }^{33 \mathrm{c}}$ which is attributed to thermal population of the higher energy screw sense state, but additionally, though to a much lesser degree, to thermal broadening of the bands as a result of slight bond length increase (the molar absorptivities for UV and $\mathrm{CD}$ spectra decrease with increasing temperature).

A temperature cycling experiment comprising three cycles of $-70 / 50^{\circ} \mathrm{C}$ with $\mathrm{CD}$ and UV spectra recorded at each temperature for $\mathbf{6 3}$ indicated that the transition is reversible, with negative and positive Cotton effects being observed at the low and high temperatures, respectively, as shown in Figure 41.

Despite the fact that the poly(diarylsilane)s are amongst the most robust polysilanes, indefinite cycling would not be possible, since the polymers are photosensitive and irradiation causes photodegradation by silicon-silicon bond scission. Excluding CD results, the data for the homologous copolymer 64 with a ratio of chiral/achiral $=0.5 / 0.5$ are similar to those of $\mathbf{6 3}$. Copolymer 65 (chiral/achiral $=0.8 / 0.2)$ with lower $M_{\mathrm{w}}$ has slightly lower UV molar absorptivity but is otherwise also comparable to $\mathbf{6 3}$, indicating similar polymer structures for 63-65. The CD data, however, are markedly different: whereas $\mathbf{6 3}$ shows negative Cotton effects at low temperature and positive at high temperature, indicating a transition from one helical screw sense to the other, both $\mathbf{6 4}$ and $\mathbf{6 5}$, and the related homopolymer, 57, show only negative Cotton effects at all temperatures and thus do not exhibit this phenomenon, i.e., this function is specific to the composition and structure of $\mathbf{6 3}$.
It is apparent, therefore, that a subtle interplay of factors underlies such a $P M$ transition, presumably as a consequence of the enthalpy and entropy terms in the Gibbs free energy being of the same sign. The sign of the free energy difference changes as a function of temperature, as was discussed for the origin of $P M$ transitions for certain polyisocyanates and poly(dialkylsilane)s. ${ }^{18 \mathrm{~b}, 18 \mathrm{~m}, 18 \mathrm{n}, 30 \mathrm{a}-30 \mathrm{c}, 30 \mathrm{~g}}$ In the present case, since both $P$ - and $M$-screw senses coexist in these polymer systems, the transition may be considered to reflect the gradual thermodynamic stabilization of one screw sense relative to the other, as the populations alter with temperature, rather than an abrupt switch. It should be noted that the energy potential curve with multi well energy minima is of primary importance in the consideration of these polymers, as well as for the poly(dialkylsilanes)s mentioned above.

\section{Chiroptical Property of Aggregates}

Aggregates-micro-precipitates of tens to thousands of polymer molecules-are formed for the poly(diarylsilane)s when a poor solvent such as methanol is added to a molecularly disperse solution of the polymer in a good solvent, such as toluene or THF. Aggregates can be invisible to the naked eye or appear as a faint cloudiness in the solution and are amenable to study by many solution techniques such as solution state CD and UV spectroscopy. Since aggregates are the first stage of a solid precipitate, they are also therefore useful as model materials of the solid bulk state polymers. Here we describe briefly our initial investigations of poly (diarylsilane) aggregates. ${ }^{57}$

The UV and CD spectra of $\mathbf{5 5}$ in toluene after addition of methanol are shown in Figure 40. These spectra are typical for those of poly(diarylsilane)s under such conditions. The aggregate UV spectrum of $\mathbf{5 5}$ is similar to the homogeneous solution spectrum, although often the intensity of the absorption is reduced and absorption tails into the visible as some light is effectively 


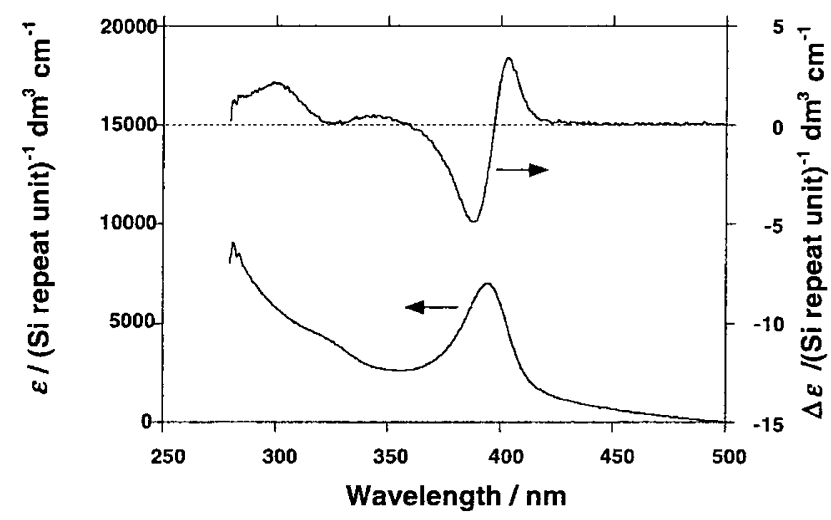

Figure 42. Aggregates of $\mathbf{5 5}$ in toluene/MeOH; upper curves $\mathrm{CD}$, lower curves UV.

prevented from reaching the detector due to scattering, resulting in non-flat baselines. The aggregate $\mathrm{CD}$ spectrum, however, is markedly different from the homogeneous solution spectrum and instead of a single Cotton effect of positive or negative sign coincident with the UV spectrum, a bisignate Cotton effect is produced as a result of coupled excitonic interactions (Davidov coupling). By convention, a bisignate Cotton effect takes the sign of the longer wavelength extremum; thus in Figure 42, the bisignate Cotton effects are positive.

The poly(diarylsilane) aggregate $\mathrm{CD}$ and UV appearances are similar to those for the poly[alkyl(alkoxyphenyl)silane]s, although shifted to longer wavelength due to the presence of two phenyl rings. However, while the latter contain an oxygen atom, allowing effective interaction with solvents and guest molecules, the former do not, and are thus less responsive to their environment.

\section{Circularly Polarized Photoluminescence}

Photoluminescence (PL) in the polysilanes is well documented, ${ }^{35,39}$ and for the poly(diarylsilane)s occurs typically with a small Stokes shift and almost mirror image profile of the UV absorption. ${ }^{33 a, 33 b, 76}$ This is due to the similarity of the chromophore and fluorophore structures in the ground and excited states, respectively, which is a result of the fact that little main chain conformational change occurs on excitation of the electrons from the $\sigma$ to the $\sigma^{*}$ orbitals. As PL is the emissive counterpart to UV, the emissive counterpart to $\mathrm{CD}$ is circularly polarized photoluminescence (CPPL). ${ }^{27 a, 43}$ Where the fluorophore is chiral, then the photo-excited state can return to the ground state with emission of circularly polarized light, the direction of polarization of which depends on the relative intensities of the righthanded and left-handed emissions $\left(I_{\mathrm{R}}\right.$ and $I_{\mathrm{L}}$, respectively), which in turn depends on the chirality of the material, or more accurately, the chirality of the photoexcited state of the material. ${ }^{43}$ Preliminary investiga- tions $^{78}$ showed that CPPL can indeed be observed in poly(diarylsilane)s, although the intensity is low.

In our experiment, an aggregate phase of $\mathbf{5 5}$ in toluene and methanol $(1: 1)$ at $20^{\circ} \mathrm{C}$ was prepared by the normal addition method and the CPPL emission spectrum was measured while irradiating at $350 \mathrm{~nm}$, on the higher frequency side of the positive/negative extremum at $395 \mathrm{~nm}$. The CPPL $\lambda_{\max }$ occurs at $412 \mathrm{~nm}$, matching the emission peak and is negative in sign. The luminescence dissymmetric ratio, glum, is approximately $-1.8 \times 10^{-3}$ for $\mathbf{5 5},{ }^{78}$ which is similar in magnitude to the $g_{\text {abs }}$ value $\left(1.4 \times 10^{-3}\right)$ for CD in UV at $390 \mathrm{~nm}$, indicating similar helical characteristics of the polymer in excited and ground states. This constitutes the first observation of CPPL for a polysilane and is a promising indicator of the possibility of circularly polarised electroluminescent (CPEL) emission from these polymers.

\section{CHIRAL SUPRAMOLECULAR STRUCTURES}

The formation of helical or chiral supramolecular structures made up of synthetic polymer building blocks has been growing interest in supramolecular chemistry and nanomaterial science. The first polymeric superstructure was reported by Nolte and collaborators in $1998 .^{79}$ They designed amphiphilic block copolymers, which constitute of flexible hydrophobic polystyrene and rigid hydrophilic dipeptidederived polyisocyanides. These CD data and TEM images revealed that the helical screw and pitch greatly depend on a proportion between polyisocyanide and polystyrene in the block copolymers, as well as an aqueous environment with $\mathrm{pH}$. They considered that transfer of chirality occurs from the amino acid in the isocyanide monomer to the secondary helical structure of the polyisocyanide blocks, leading to the helical superstructure upon assembly.

Moreover, they developed the idea to multiblock copolymers, 66, constituting a rather polydisperse, optically inactive poly (methylphenylsilane) $\left(M_{\mathrm{n}}=4400\right.$, $\left.M_{\mathrm{w}} / M_{\mathrm{n}}=1.6\right)$ and poly(ethylene oxide) $\left(M_{\mathrm{n}}=7000\right.$, $\left.M_{\mathrm{w}} / M_{\mathrm{n}}=1.03\right) .{ }^{80}$ They demonstrated the formation of vesicles in pure water and the tunability of aggregation behavior by modifying ratios of water/THF cosolvents. TEM images revealed that the micellar fiber rods formed in mixtures of water/THF between $40 / 60$ and $80 / 20 \%(\mathrm{v} / \mathrm{v})$. In addition, helical supramolecular structures with left- and right-handed screw senses (superhelix) produced in a mixture of water/THF of 90/10\% (v/v) from TEM and SEM observation. They considered that the initial poly(methylphenylsilane) blocks cooperatively determine the handedness of the 


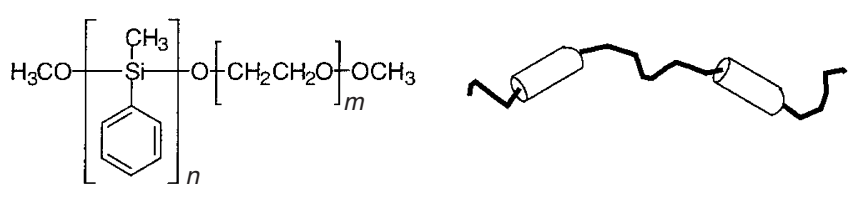

66

Chart 14. Chemical structure of block copolymers, 66, consisting of hydrophobic poly(methylphenylsilane) segments and felxible, hydrophylic poly(ethylene oxide) segments.

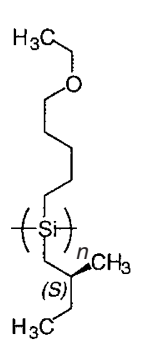

67

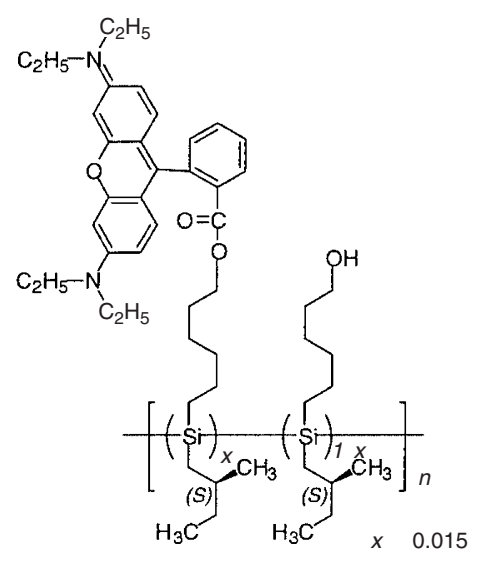

68

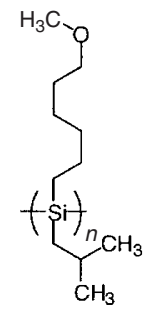

69
Chart 15. Chemical structure of rodlike polysilanes with LB film forming ability.

subsequent poly(methylphenylsilane) blocks assembling in the same handedness of aggregate, resulting in several chiral architectures. ${ }^{80}$ As desribed in previously, because poly(methylphenylsilane) with latent helicity may have a dynamic twisting helical main chain with equal proportion of $P$ - and $M$-main chain motifs, no preferential screw sense ordering might be responsible for the both left- and right-handed superhelices.

\section{OTHER OPTICALLY INACTIVE, HELICAL POLYSILANES AND LANGMUIR-BLODGET FILM}

Tokura and coworkers prepared rodlike helical optically active polysilane homopolymer containing ether side group (67). ${ }^{81 a}$ They demonstrtaed an introduction of ether moiety to polysilane improves LangmuirBlodget (LB) film forming ability and achieved highly oriented polysilane LB film by thermal anealing treatment of pristine film at $100^{\circ} \mathrm{C}$. The dichroic ratio increases up to 9, twice that for the as-deposited LB film, with the annealing time and tends to saturate over $12 \mathrm{~h}$.

They also prepared 67-related copolymer bearing few $\%$ of organic fluorophore $(\mathbf{6 8}) .{ }^{81 \mathrm{~b}}$ It was observed that excitation energy is transferred efficiently by free excitons on the Si backbones to reach the luminescence centers, where energy transfer occurs from the exciton to the dye molecule. The exciton transfer occurred over 50-100 monomer units on the backbone. This approach may facilitate to fabricating a wavelength-tunable luminous device by utilizing the exciton transport on $\mathrm{Si}$ chains.

Similarly, Yoshida and coworkers also prepared rodlike helical optically inactive polysilane homopolymer containing ether side group (69) and studied an monolayer forming ability of this at the air/water interface. ${ }^{81 \mathrm{c}, 81 \mathrm{~d}}$ They found that polysilane monolayers demonstrated a unique chromic effect induced by a specific conformational and orientational change of polymer backbones at the air/water interface.

\section{CONCLUDING REMARKS}

Amongst numbers of optically active synthetic polymers, optically active polysilanes exhibit unique absorption, circular dichroism, and fluorescence spectra around $300-400 \mathrm{~nm}$ due to $\sigma$-conjugation. Since the first brief report concerning optically active polysilanes in 1992, the field of optically active polysilane homo- and copolymers has grown and evolved to the present. In this review, we have discussed the synthesis, chiroptical properties, and characterization of homo- and copolymers of optically active poly(dialkylsilane)s, poly(dialkoxysilane)s, poly[alkyl(aryl)silane]s, and poly(diarylsilane)s. This review describes comprehensively (i) the mutual relationship between side chain structure, (ii) global shapes and local structures, (iii) (chir)optical properties, (iv) (semi)quantitative population analysis of right- and left-handed helices based on Kuhn's dissymmetry ratio, (v) several helical cooperativity effects, (vi) molecular imaging, (vii) inversion of screw-sense, (viii) chiroptical switch and memory, (ix) transfer and amplification of molecular chirality to aggregates, (x) cholesteric liquid crystallinity, (xi) helical supramolecular structures, and (xii) latent helicity, as consequences of side group internal interactions and other external stimuli. These advances should contribute to optically active polymer research in the areas of traditional polymer science and engineering, and also in the applied areas of nanomaterial science and nanotechnology at the sub-nm level in the future.

\section{APPENDIX. THEORETICAL ANALYSIS OF CONFORMATIONAL TRANSITIONS ${ }^{18 \mathrm{i}, 18 \mathrm{j}, 18 \mathrm{o}, 31 \mathrm{~d}}$}

However, the discussion up to above is based on an oversimplified view of the polymer conformation, 
categorizing it into the three discrete regions. To be more precise, the conformation of a polymer $\mathbf{2 4}$ chain changes continuously from pure $P$ - to pure $M$-motifs with temperature and solvent as shown in Figure A1. ${ }^{31 \mathrm{~d}}$ Here the transition is very sharp at high molecular weight, but progressively broadens as the number of $\mathrm{Si}$ atoms/chain, $N_{\mathrm{s}}$, is decreased; the global chain conformation is rod-like throughout the transition. An interesting feature of this transition is that the transition temperature $T_{\mathrm{c}}$ of $280 \mathrm{~K}$, where $f_{\mathrm{P}}=f_{\mathrm{M}}=1 / 2$, is independent of $N_{\mathrm{s}}$. This is a characteristic feature of a linear Ising system with no restriction on the conformations of the terminal units ${ }^{12 \mathrm{e}}$ and this is the first evidence in support of this prediction. The theory ${ }^{12 \mathrm{e}}$ assumes that a polymer chain consists of an alternating array of $P$ - and $M$-helices joined by helix reversals and characterizes its conformation as a function of $N_{\mathrm{s}}$ by the two thermodynamic parameters, i.e., the free energy difference between $P$ and $M$-motifs, $2 \Delta G_{\mathrm{h}}\left(=G_{\mathrm{M}}-G_{\mathrm{P}}\right)$, and free energy of helix reversal, $\Delta G_{\mathrm{r}}$, thus representing $f_{\mathrm{P}}$ as a function of $N_{\mathrm{s}}$ with $\Delta G_{\mathrm{h}}$ and $\Delta G_{\mathrm{r}}$ adjustable parameters, or the corresponding energy and entropy, $\Delta H_{\mathrm{h}}$ and $\Delta S_{\mathrm{h}}$. With an appropriate choice of $g_{\mathrm{m}}$, the $g_{\mathrm{abs}}$ value for perfect $P$-helix, $g_{\text {abs }}$ is converted to $f_{\mathrm{P}}$, and $f_{\mathrm{P}}$ as a function of $N_{\mathrm{s}}$ at fixed temperature $T$ is compared to the theory. Such a comparison has been made successfully for the data in Figure A1, ${ }^{12 \mathrm{e}, 31 \mathrm{~d}}$ yielding $\Delta G_{\mathrm{r}}(T)$ $=1.2 \times 10^{4}\left(\mathrm{~J} \mathrm{~mol}^{-1}\right)$ and

$$
\begin{aligned}
& \Delta G_{\mathrm{h}}(T)=-3+43 \sin [-0.019 \times(T / K-280)] \\
& \quad \approx 360-1.305 T\left(\mathrm{~J} \mathrm{~mol}^{-1}\right) \\
& T_{\mathrm{c}}=\Delta H_{\mathrm{h}} / \Delta S_{\mathrm{h}}=275.86 \mathrm{~K}
\end{aligned}
$$

Indeed the solid curves in Figure A1 represent the theoretical values calculated with these parameters, fitting precisely the data points. This free energy $\Delta G_{\mathrm{h}}$ $(T)$ implies that the $P$-motif dwells in a deep but narrow energy well compared to the $M$-motif, namely positive $\Delta H_{\mathrm{h}}$ and positive $\Delta S_{\mathrm{h}}$, as shown in Figure A2. This conforms to the molecular dynamics calculation shown in Figure 18b. However the thermal transition occurs by the competition between the enthalpy and entropy terms in eq 1, which predicts such potential contours assumed in Figure 18b. The solvent dependence of $T_{\mathrm{c}}$ shown in Figure 17 is explained by the interaction of solvent molecules with the helical cavity of the polymer. Compared with stereo-specifically deuterated poly(hexyl isocyanate)s ${ }^{18 \mathrm{i}, 18 \mathrm{j}} \Delta G_{\mathrm{r}}(T)$ is significantly smaller for polymer $\mathbf{2 4}$ and polymer $\mathbf{3}$. This may be due to a relatively easy pass way from $P$ to $M$ for polymer 24, whereas this is difficult for the polyisocyanates, where the helix reversal needs the rotation about amide bonds. On the other hand $\Delta G_{\mathrm{h}}(T)$ is much larger in magnitude for polymer 24. Thus we see that the

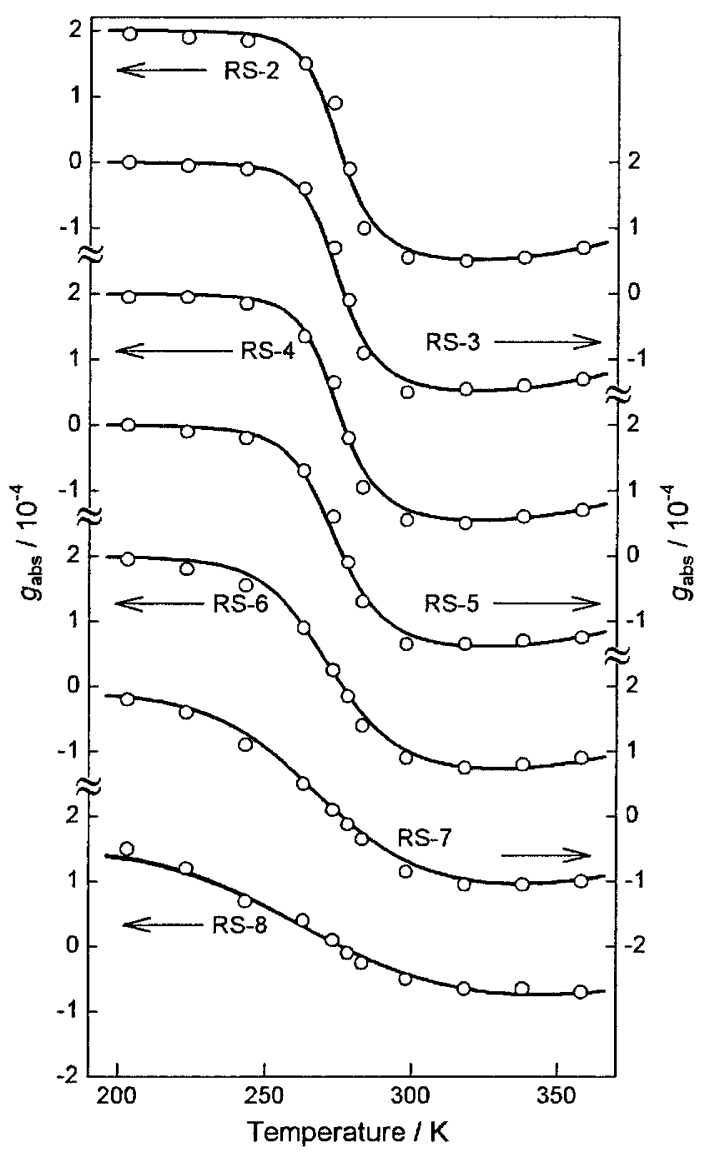

Figure A1. Temperature dependence of $g_{\text {abs }}$ for seven samples of polymer $\mathbf{2 4}$ with $N_{\mathrm{s}}$ between 35.0 and 5300 . Solid curves, theoretical values. ${ }^{31 \mathrm{~d}}$

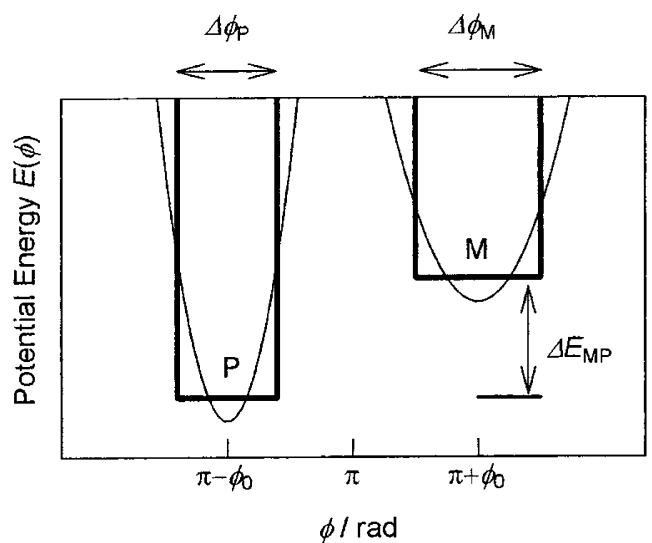

Figure A2. Schematic representation of a double-well potential: Energy difference, $\Delta E_{\mathrm{MP}}=E_{\mathrm{M}}-E_{\mathrm{P}}$; widths of wells, $\Delta \phi_{\mathrm{P}}$, $\Delta \phi_{\mathrm{M}}$.

chemical structure difference can be quantitatively transcribed to the thermodynamic parameters, which in turn may be used to seek the required structure. The molecular weight dependence of $g_{\text {abs }}$ as shown in Figure A1 is a unique feature common to all the conformational transitions of polymers in solution, ${ }^{12 \mathrm{f}}$ as also found with polymer $3,{ }^{30 \mathrm{q}}$ and the molecular weight is one of important bridges between chemistry and physics in polymer conformation. The above analysis predicts that 
the transition approaches indefinitely a first-order transition as the potentials become narrower with energy barriers bypassed with thermal energy and suggests a possible way to an ideal quantum switch.

Acknowledgments. The authors thank Hiromi Tamoto-Takigawa and Masao Motonaga for CD, UV, FL, and SEC measurements. Prof. Kyozaburo Takeda (Waseda Univ.), and Dr. Hiroyuki Teramae (ATR), Prof. Nobuo Matsumoto (Shonan Institute of Technology), Dr. Masaie Fujino, Dr. Hiroaki Isaka, Dr. Keisuke Ebata, and Dr. Kazuaki Furukawa are grateful for valuable discussions on the electronic structure and optoelectronic properties of polysilanes at NTT. Drs. Masao Morita, Kei-ichi Torimitsu, Hideaki Takayanagi, and Noriyuki Hatakenaka are acknowledged for encouragement and generous support at NTT during the course of this study.

Profs. Mark M. Green (Polytechnic Univ.) and Toyoki Kunitake (Univ. Kitakyushu) are acknowledged for inviting us into the realm of cooperative polymer science. Prof. Naotaka Nakamura (Ritsumeikan Univ.), Prof. Shoji Furukawa (Kyushu Institute of Technology), Prof. Eiji Yashima (Nagoya Univ.), Prof. Yoshihisa Inoue (Osaka Univ.), and Prof. Masashi Kunitake (Kumamoto Univ.) are gratefully acknowledged for their insight on the global shape in solution and origin of the helix-helix transition.

Also, Profs. Shin-ya Koshihara (Tokyo Institute of Technology), Mamoru Yoshimoto (Tokyo Institute of Technology), Hideomi Koinuma (Tokyo Institute of Technology), and Drs. Tatsuo Maeda, Tsuyoshi Ohnishi, Kazuaki Furukawa, and Keisuke Ebata are thanked for collaboration on the AFM imaging of polysilanes. Prof. Tsuneki Ichikawa (Hokkaido Univ.), Dr. Jun Kumagai (Nagoya Univ.) and Dr. Yoshihisa Yamada (Hokkaido Univ.) are thanked for collaboration on the degree of delocalization for hole and electron carriers of polysilylenes. Drs. Hong-Zhi Tang (the former CREST-JST, now Univ. Washington) and ZhongBiao Zhang (the former CREST-JST, now Univ. South California) are thanked for stimulating discussions on the optically activity of polysilanes. Dr. Kento Okoshi, Hiroyuki Kamee, and Tomoko Natume are grateful for collaboration on the liquid crystalline properies of rodlike polysilanes.

Profs. R. J. M. Nolte (Univ. Nijmegen), R. G. Jones (Univ. Kent), and N. A. J. M. Sommerdijk (Eindhoven Univ. Technology) are acknowledged for informing the formation of superstructures based on polysilane-PEO block copolymers.

Prof. Hideki Sakurai (Science Univ. of Tokyo), Prof. Masaki Hasegawa (Toin Univ. of Yokohama), and
Dr. Katsuhiko Kuroda (CREST-JST) are acknowledged for their encouragement, and Yukiko Shimamura and Ayako Nagano (CREST-JST) for able assistance. Finally, Profs. Yoshio Okamoto (Nogoya Univ.) and Yotaro Morishima (the former Osaka Univ., now Fukui Univ. of Technology) are acknowledged for inviting the present review paper.

\section{REFERENCES}

1. a) S. F. Mason, Nature, 311, 19 (1984).

b) S. F. Mason, "Chemical Evolution: Origin of the Elements, Molecules, and Living Systems", 2nd ed., Oxford, University Press, Inc., London, 1991.

c) S. F. Mason, in "Circular Dichroism: Principles and Applications", 2nd ed, N. Berova, K. Nakanishi, and R. W. Woody, Ed., Wiley-VCH, New York, N.Y., 2000, chapt. 2.

2. a) W. A. Bonner, P. R. Kavasmaneck, F. S. Martin, and J. J. Flores, Science, 186, 143 (1974).

b) W. A. Bonner, Chirality, 12, 114 (2000).

3. a) M. Avalos, R. Babiano, P. Cintas, J. L. Jiménez, and J. C. Palacios, Tetrahedron: Asymmetry, 8, 2997 (1997).

b) M. Avalos, R. Babiano, P. Cintas, J. L. Jiménez, J. C. Palacios, and L. D. Barron, Chem. Rev., 98, 2391 (1998).

c) M. Hasegawa, Proc. Jpn. Acad. Ser. B, 68, 9 (1992).

4. a) A. Yamagata, J. Theoret. Biol., 11, 495 (1966).

b) R. A. Harris and L. Stodolsky, Phys. Lett., Sect. B, 78, 313 (1978).

c) D. K. Kondepudi and G. W. Nelson, Nature, 314, 438 (1985).

d) R. A. Hegstrom, Nature, 315, 749 (1985).

e) M. Quack, Chem. Phys. Lett., 132, 1471 (1986).

f) L. Wiesenfeld, Mol. Phys., 64, 739 (1988).

g) A. Salam, J. Mol. Evol., 33, 105 (1991).

h) R. Berger and M. Quack, Chem. Phys. Chem., 1, 57 (2000).

i) R. A. Harris, Chem. Phys. Lett., 365, 343 (2002).

5. a) D. B. Cline, Ed., "Physical Origin of Homochirality in Life", American Institute of Physics, Woodbury, N.Y., 1996.

b) M. Gardner, Ed., "The New Ambidextrous UniverseSymmetry and Asymmetry from Mirror Reflections to Superstrings", 3rd rev. ed., W. H. Freeman, New York, N.Y., 1990. c) H. Latel, in "Chirality-From Weak Bosons to the $\alpha$-Helix", R. Janoschek Ed., Springer-Verlag GmbH\&Co., Berlin, 1991, chapt. 1.

6. For reviews on vinyl polymers and molecular helicity.

a) M. Farina, Top. Stereochem., 17, 1 (1987).

b) K. P. Meurer and F. Vögtle, Top. Curr. Chem., 127, 1 (1985).

7. For reviews on poly(triphenylmethyl methacrylate).

a) Y. Okamoto and T. Nakano, Chem. Rev., 94, 349 (1994).

b) T. Nakano and Y. Okamoto, Macromol. Rapid Commun., 21, 603 (2000).

8. R. J. M. Nolte, Chem. Soc. Rev., 23, 11 (1994).

9. For reviews and book of optically activity induction in polyisocyanates.

a) M. M. Green, N. C. Peterson, T. Sato, A. Teramoto, R. Cook, and S. Lifson, Science, 268, 1860 (1995).

b) M. M. Green, J.-W. Park, T. Sato, A. Teramoto, S. Lifson, 
R. L. B. Selinger, and J. V. Selinger, Angew. Chem. Int. Ed., 38, 3138 (1999).

c) M. M. Green, in "Circular Dichroism: Principles and Applications", 2nd ed, N. Berova, K. Nakanishi, and R. W. Woody, Ed., Wiley-VCH, New York, N.Y., 2000, chapter 17. d) M. M. Green, K-P. Cheon, S-Y. Yang, J-W. Park, S. Swanburg, and W. Liu, Acc. Chem. Res., 34, 672 (2001).

10. For a book of induced optical activity in polyacetylenes. E. Yashima and Y. Okamoto, in "Circular Dichroism: Principles and Applications", 2nd ed, N. Berova, K. Nakanishi, and R. W. Woody, Ed., Wiley-VCH, New York, N.Y., 2000, chapter 18 .

11. For a review of optically active chromophoric polymers. L. Pu, Acta Polym., 48, 116 (1997).

12. For reviews of helical polymer characterization.

a) O. Kratky and G. Porod, Recl. Trav. Chim., 68, 1106 (1949).

b) T. Norisuye, T. Yanaki, and H. Fujita, J. Polym. Sci., polym. Phys. Ed., 18, 547 (1980).

c) H. Murakami, T. Norisuye, and H. Fujita, Macromolecules, 13, 345 (1980).

d) T. Norisuye, Prog. Polym. Sci., 18, 543 (1993).

e) H. Yamakawa, Ed. "Helical Wormlike Chains in Polymer Solutions", Springer-Verlag GmbH\&Co., Berlin, 1997.

f) A. Teramoto, Prog. Polym. Sci., 26, 667 (2001).

13. a) M. Goodman and S.-C. Chen, Macromolecules, 3, 398 (1970).

b) M. Goodman and S.-C. Chen, Macromolecules, 4, 625 (1971).

14. a) F. Millich and G. K. Baker, Macromolecules, 2, 122 (1969). b) R. J. M. Nolte, A. J. M. van Beijnen, and W. Drenth, J. Am. Chem. Soc., 96, 5932 (1974).

c) A. J. M. van Beijnen, R. J. M. Nolte, W. Drenth, and A. M. F. Hezemans, Tetrahedron, 32, 2017 (1976).

15. a) P. Pino and G. P. Lorenzi, J. Am. Chem. Soc., 82, 4745 (1960).

b) P. Pino, F. Ciardelli, G. Montagnoli, and O. Pieroni, J. Polym. Sci., Polym. Lett. Ed., 5, 307 (1967).

c) C. Carlini, P. Pino, and F. Ciardelli, Makromol. Chem., 119, 244 (1968).

16. Y. Okamoto, K. Suzuki, K. Ohta, K. Hatada, and H. Yuki, J. Am. Chem. Soc., 101, 4768 (1979).

17. a) P. C. J. Kamer, M. C. Cleij, R. J. M. Nolte, T. Harada, A. M. F. Hezemans, and W. Drenth, J. Am. Chem. Soc., 110, 1581 (1988).

b) M. M. Green, R. A. Gross, F. C. Schilling, K. Zero, and C. C. Crosby III, Macromolecules, 21, 1839 (1988).

c) T. J. Deming and B. M. Novak, J. Am. Chem. Soc., 114, 7926 (1992).

d) Y. Ito, E. Ihara, and M. Murakami, Angew. Chem. Int. Ed. Engl., 31, 1509 (1992).

e) M. Kauranen, T. Verbiest, C. Boutton, M. N. Teerenstra, K. Clays, A. J. Schouten, R. J. M. Nolte, and A. Persoons, Science, 270, 966 (1995).

f) F. Takei, K. Yanai, K.Onitsuka, and S. Takahashi, Angew. Chem. Int. Ed. Engl., 35, 1554 (1996).

g) Y. Itoh, T. Ohara, R. Shima, and M. Suginome, J. Am. Chem. Soc., 118, 9188 (1996).

h) E. Ramos, J. Bosch, J.-L. Serrano, T. Sierra, and J. Veciana,
J. Am. Chem. Soc., 118, 4703 (1996).

i) D. B. Amabilino, E. Ramos, J.-L. Serrano, T. Sierra, and J. Veciana, J. Am. Chem. Soc., 120, 9126 (1998).

j) Y. Ito, T. Miyake, S. Hatano, R. Shima, T. Ohara, and M. Suginome, J. Am. Chem. Soc., 120, 11880 (1998).

k) F. Takei, H. Hayashi, K. Onitsuka, N. Kobayashi, and S. Takahashi, Angew. Chem. Int. Ed. Engl., 41, 4092 (2001).

1) F. Takei, H. Hayashi, K. Onitsuka, and S. Takahashi. Polym. J., 33, 310 (2001).

m) F. Feng, T. Miyashita, F. Takei, K. Onitsuka, and S. Takahashi, Chem. Lett., 764 (2001).

n) J. J. L. M. Cornelissen, J. J. J. M. Donners, R. de Gelder, W. S. Graswinckel, G. A. Metselaar, A. E. Rowan, N. A. J. M. Sommerdijk, and R. J. M. Nolte, Science, 293, 676 (2001).

o) J. J. L. M. Cornelissen, W. S. Graswinckel, P. J. H. M. Adams, G. H. Nachtegaal, A. P. M. Kentgens, N. A. J. M. Sommerdijk, and R. J. M. Nolte, J. Polym. Sci., Part A: Polym. Chem., 39, 4255 (2001).

p) Y. Yamada, T. Kawai, J. Abe, and T. Iyoda, J. Polym. Sci., Part A: Polym. Chem., 40, 399 (2002).

18. a) M. M. Green, C. Andreola, B.Muñoz, M. P. Reidy, and K. Zero, J. Am. Chem. Soc., 110, 4043 (1988).

b) M. M. Green, M. P. Reidy, R. J. Johnson, G. Darling, D. J. O'Leary, and G. Willson, J. Am. Chem. Soc., 111, 6452 (1989).

c) Y. Okamoto, M. Matsuda, T. Nakano, and E. Yashima, Polym. J., 25, 391 (1993).

d) M. M. Green, C. Khatri, and N. C. Peterson, J. Am. Chem. Soc., 115, 4941 (1993).

e) M. Müller and R. Zentel, Macromolecules, 27, 4404 (1994).

f) G. Maxein and R. Zentel, Macromolecules, 28, 8438 (1995).

g) K. Maeda, M. Matsuda, T. Nakano, and Y. Okamoto, Polym. J., 27, 141 (1995).

h) M. M. Green, B. A. Garetz, B. Munoz, and H.-P. Chang, J. Am. Chem. Soc., 117, 4181 (1995).

i) M. Müller and R. Zentel, Macromolecules, 29, 1609 (1996).

j) J. M. Guenet, H. S. J. Jeon, C. Khatri, S. K. Jha, N. P. Balsara, M. M. Green, A. Brulet, and A. Thierry, Macromolecules, 30, 4590 (1997).

k) C. A. Khatri, Y. Pavlova, M. M. Green, and H. Morawetz, J. Am. Chem. Soc., 119, 6991 (1997).

1) S. Mayer, G. Maxein, and R. Zentel, Macromolecules, 31, 8522 (1998).

m) K. Maeda and Y. Okamoto, Macromolecules, 31, 5164 (1998).

n) K. Maeda and Y. Okamoto, Macromolecules, 31, 1046 (1998).

o) S. Mayer, G. Maxein, and R. Zentel, Macromolecules, 31, 8522 (1998).

p) K. Maeda and Y. Okamoto, Macromolecules, 32, 974 (1999).

q) S. K. Jha, K.-S. Cheon, M. M. Green, and J. V. Selinger, J. Am. Chem. Soc., 121, 1665 (1999).

r) K. Ute, Y. Fukunishi, S. K. Jha, K.-S. Cheon, B. Munoz, K. Hatada, and M. M. Green, Macromolecules, 32, 1304 (1999). s) H. Gu, Y. Nakamura, T. Sato, A. Teramoto, M. M. Green, and C. Andreola, Polymer, 40, 849 (1999). 
t) K. S. Cheon, J. V. Selinger, and M. M. Green, Angew. Chem. Int. Ed., 39, 1482 (2000).

u) J. Li, G. B. Schuster, K.-S. Cheon, M. M. Green, and J. V. Selinger, J. Am. Chem. Soc., 122, 2603 (2000).

19. a) O. Pieroni, F. Matera, and F. Ciardelli, Tetrahedron Lett., 7, 597 (1972).

b) F. Ciardelli, S. Lanzillo, and O. Pieroni, Macromolecules, 7, 174 (1974).

c) J. S. Moore, C. B. Gorman, and R. H. Grubbs, J. Am. Chem. Soc., 113, 1704 (1991).

d) E. Yashima, T. Matsushima, and Y. Okamoto, J. Am. Chem. Soc., 117, 11596 (1995).

e) E. Yashima, T. Nimura, T. Matsushima, and Y. Okamoto, J. Am. Chem. Soc., 118, 9800 (1996).

f) E. Yashima, T. Matsushima, and Y. Okamoto, J. Am. Chem. Soc., 119, 6345 (1997).

g) E. Yashima, K. Maeda, and Y. Okamoto, J. Am. Chem. Soc., 120, 8895 (1998).

h) E. Yashima, K. Maeda, and Y. Okamoto, Nature, 399, 449 (1999).

i) H. Nakako, R. Nomura, M. Tabata, and T. Masuda, Macromolecules, 32, 2861 (1999).

j) T. Aoki, Y. Kobayashi, T. Kaneko, E. Oikawa, Y. Yamamura, Y. Fujita, M. Teraguchi, R. Nomura, and T. Masuda, Macromolecules, 32, 79 (1999).

k) R. Nomura, Y. Fukushima, H. Nakako, and T. Masuda, $J$. Am. Chem. Soc., 122, 8830 (2000).

1) H. Nakako, Y. Mayahara, R. Nomura, M. Tabata, and T. Masuda, Macromolecules, 33, 3978 (2000).

m) H. Nakako, R. Nomura, and T. Masuda, Macromolecules, 34, 1496 (2001).

n) R. Nomura, J. Tabei, and T. Masuda, J. Am. Chem. Soc., 123, 8430 (2001).

o) H. Onouchi, K. Maeda, and E. Yashima, J. Am. Chem. Soc., 123, 7441 (2001).

p) E. Yashima, K. Maeda, and O. Sato, J. Am. Chem. Soc., 123, 8159 (2001).

q) E. Yashima, Anal. Sci., 18, 3 (2002).

r) A. P. H. J. Schenning, M. Fransen, and E. W. Meijer, Macromol. Rapid Commun., 23, 265 (2002).

20. a) M. Lemaire, D. Delabouglise, R. Garreau, A. Guy, and J. Roncali, J. Chem. Soc., Chem. Commun., 658 (1988).

b) M. Andersson, P. O. Ekeblad, T. Hjertberg, O. Wennerström, and O. Inganäs, Polym. Commun., 32, 546 (1991).

c) M. M. Bouman and E. W. Meijer, Adv. Mater., 7, 385 (1995).

d) G. Bidan, S. Guillerez, and V. Sorokin, Adv. Mater, , 8, 157 (1996).

e) B. M. W. Langeveld-Voss, R. A. J. Janssen, M. P. T. Christiaans, S. C. J. Meskers, H. P. J. M. Dekkers, and E. M. Meijer, J. Am. Chem. Soc., 118, 4908 (1996).

f) F. Andreani, L. Angiolini, D. Caretta, and E. Salatelli, J. Mater. Chem., 8, 1109 (1998).

g) E. R. Lermo, B. M. W. Langeveld-Voss, R. A. J. Janssen, and E. W. Meijer, Chem. Commun., 791 (1999).

h) E. Yashima, H. Goto, and Y. Okamoto, Macromolecules, 32, 7942 (1999).

i) B. M. W. Langeveld-Voss, R. J. M. Waterval, R. A. J. Janssen, and E. W. Meijer, Macromolecules, 32, 227 (1999). j) Z.-B. Zhang, M. Fujiki, M. Motonaga, H. Nakashima, K. Torimitsu, and H.-Z. Tang, Macromolecules, 35, 941 (2002).

21. a) E. Peeters, M. P. T. Christiaans, R. A. J. Janssen, H. F. M. Schoo, H. P. J. M. Dekkers, and E. W. Meijer, J. Am. Chem. Soc., 119, 9909 (1997).

b) E. Peeters, A. Delmotte, R. A. J. Janssen, and E. W. Meijer, Adv. Mater, 9, 493 (1997).

22. a) A. Goodwin and B. M. Novak, Macromolecules, 27, 5520 (1994).

b) M.-P. Nieh, A. A. Goodwin, J. R. Stewart, B. M. Novak, and D. A. Hoagland, Macromolecules, 31, 3151 (1998).

c) D. S. Schiltzer and B. M. Novak, J. Am. Chem. Soc., 120, 2196 (1998).

d) A. M. Heintz and B. M. Novak, Polym. Prepr. (Am. Chem. Soc., Div. Polym. Chem.), 39(2), 429 (1998).

23. a) J.-H. Fuhrhop, P. Blumtritt, C. Lehmann, and P. Luger, $J$. Am. Chem. Soc., 113, 7437 (1991).

b) D. A. Frankel and D. F. O'Brien, J. Am. Chem. Soc., 116, 10057 (1994).

c) J. M. Schnur, B. R. Ratna, J. V. Selinger, A. Singh, G. Jyothi, and K. R. K. Easwaran, Science, 264, 945 (1994).

d) A. F. Drake, P. Udvarhelyi, D. J. Ando, D. Bloor, J. S. Obhi, and S. Mann, Polymer, 30, 1043 (1989).

24. a) M. Salmon and G. Bidan, J. Electrochem. Soc., 132, 1897 (1985).

b) D. Delabouglise and F. Garnier, Synth. Met., 39, 117 (1990).

25. a) M. R. Majidi, L. A. P. Kane-Maguire, and G. G. Wallace, Polymer, 35, 3113 (1994).

b) P. C. Innis, I. D. Norris, L. A. P. Kane-Maguire, and G. G. Wallace, Macromolecules, 31, 6521 (1995).

c) S.-J. Su and N. Kuramoto, Chem. Lett., 504 (2001).

d) S.-J. Su and N. Kuramoto, Macromolecules, 34, 7249 (2001).

26. R. Fiesel and U. Scherf, Acta Polym., 49, 445 (1998).

27. a) M. Oda, H.-G. Nothofer, G. Lieser, U. Scherf, S. C. Meskers, and D. Neher, Adv. Mater, 12, 362 (2000).

b) M. Oda, S. C. J. Meskers, H. G. Nothofer, U. Scherf, and D. Neher, Synth. Met., 111, 575 (2000).

c) H.-Z. Tang, M. Fujiki, and T. Sato, Macromolecules, 35, 6439 (2002).

d) M. Leclerc, J. Polym. Sci., A: Polym. Chem., 39, 2867 (2001).

e) D. Neher, Macromol. Rapid Commun., 22, 1365 (2001).

f) H.-Z. Tang, M. Fujiki, and M. Motonaga, Polymer, 43, 6213 (2002).

28. a) J.-M. Lehn, A. Rigault, J. Siegel, J. Harrowfield, B. Chevrier, and D. Moras, Proc. Natl. Acad. Sci. U.S.A., 84, 2565 (1987).

b) U. Koert, M. M. Harding, and J.-M. Lehn, Nature, 346, 339 (1990).

c) E. C. Constable, Angew. Chem., Int. Ed. Engl., 30, 1450 (1991).

d) Y. Dai, T. J. Katz, and D. A. Nichols, Angew. Chem., Int. Ed. Engl., 35, 2109 (1996).

e) T. Takata, Y. Furusho, K.-I. Murakawa, T. Endo, H. Matsuoka, T. Hirasa, J. Matsuo, and M. Sisido, J. Am. Chem. Soc., 120, 4530 (1998).

f) A. Williams, Chem. Eur. J., 3, 15 (1997). 
g) T. Yokozawa, R. B. Prince, and J. S. Moore, J. Am. Chem. Soc., 121, 2643 (1999).

h) R. Fiesel, C. E. Halkyard, M. E. Rampey, L. Kloppenburg, S. L. Studer-Martinez, U. Scherf, and U. H. F. Bunz, Macromol. Rapid Commun., 20, 107 (1999).

i) E. Yashima, K. Maeda, and T. Yamanaka, J. Am. Chem. Soc., 122, 7813 (2000).

j) R. B. Prince, L. Brunsveld, E. W. Meijer, and J. S. Moore, Angew. Chem., Int. Ed., 39, 228 (2000).

29. For reviews on chiral polymers including polypeptides,

a) P. Urnes and P. Doty, Adv. Protein Chem., 16, 401 (1961).

b) "Poly- $\alpha$-Amino Acids", G. D. Fasman, Ed., Marcel Dekker, New York, N.Y. 1967.

c) A. Teramoto and H. Fujita, Adv. Polym. Sci., 18, 65 (1975).

d) A. Teramoto and H. Fujita, J. Macromol. Sci., Rev. Macromol. Chem., C15, 165 (1976).

e) A. Chakrabartty and R. L. Baldwin, Adv. Protein Chem., 46, 141 (1995).

f) T. Nakano and Y. Okamoto, Chem. Rev., 101, 4013 (2001). g) E. Lacroix, A. R. Viguera, and L. Serrano, J. Mol. Biol., 284, 173 (1998).

h) J. J. L. M. Cornelissen, A. E. Rowan, R. J. M. Nolte, and N. A. J. M. Sommerdijk, Chem. Rev., 101, 4039 (2001).

30. For optically active poly(dialkylsilane)s,

a) K. Matyjaszewski, J. Inorg. Organomet. Polym., 2, 5 (1992).

b) H. Frey, M. Möller, and K. Matyjaszewski, Macromolecules, 27, 1814 (1994).

c) H. Frey, M. Möller, A. Turetskii, B. Lots, and K. Matyjaszewski, Macromolecules, 28, 5498 (1995).

d) M. Fujiki, J. Am. Chem. Soc., 116, 6017 (1994).

e) M. Fujiki, J. Am. Chem. Soc., 116, 11976 (1994).

f) M. Fujiki, Appl. Phys. Lett., 65, 3251 (1994).

g) M. Fujiki, Polym. Prepr. (Am. Chem. Soc., Div. Polym. Sci.), 37(2), 454 (1996).

h) K. Obata, C. Kabuto, and M. Kira, J. Am. Chem. Soc., 119, 11345 (1997).

i) K. Shinohara, T. Aoki, T. Kaneko, and E. Oikawa, Chem. Lett., 361 (1997).

j) K. Obata and M. Kira, Macromolecules, 31, 4666 (1998).

k) M. Fujiki, S. Toyoda, C.-H. Yuan, and H. Takigawa, Chirality, 10, 667 (1998).

1) T. Ichikawa, Y. Yamada, J. Kumagai, and M. Fujiki, Chem. Phys. Lett., 306, 275 (1999).

m) K. Terao, T. Terao, A. Teramoto, N. Nakamura, I. Terakawa, T. Sato, and M. Fujiki, Macromolecules, 34, 2682 (2001)

n) J. Watanabe, H. Kamee, and M. Fujiki, Polym. J., 33, 495 (2001).

o) K. Terao, Y. Terao, A. Teramoto, N. Nakamura, M. Fujiki, and T. Sato, Macromolecules, 34, 4519 (2001).

p) K. Terao, Y.-I. Terao, A. Teramoto, N. Nakamura, M. Fujiki, and T. Sato, Macromolecules, 34, 6519 (2001).

q) T. Natsume, L. Wu, T. Sato, K. Terao, A. Teramoto, and M. Fujiki, Macromolecules, 34, 7899 (2001).

r) T. Sanji, T. Takase, and H. Sakurai, J. Am. Chem. Soc., 123, 12690 (2001).

s) T. Sato, K. Terao, A. Teramoto, and M. Fujiki, Macromolecules, 35, 2141 (2002). t) K. Okoshi, H. Kamee, G. Suzaki, M. Tokita, M. Fujiki, and J. Watanabe, Macromolecules, 35, 4556 (2002).

31. For helix-helix transition poly(dialkylsilane)s,

a) M. Fujiki, J. Am. Chem. Soc., 122, 3336 (2000).

b) M. Fujiki, Macromol. Rapid Commun., 22, 669 (2001).

c) M. Fujiki, J. R. Koe, H. Nakashima, M. Motonaga, K. Terao, and A. Teramoto, J. Am. Chem. Soc., 123, 6253 (2001). d) A. Teramoto, K. Terao, Y. Terao, N. Nakamura, T. Sato, and M. Fujiki, J. Am. Chem. Soc., 123, 12303 (2001).

e) M. Fujiki, M. Motonaga, H.-Z, Tang, K. Torimitsu, Z.-B. Zhang, J. R. Koe, J. Watanabe, K. Terao, T. Sato, and A. Teramoto, Chem. Lett., 1218 (2001).

f) M. Fujiki, H.-Z. Tang, M. Motonaga, K. Torimitsu, J. R. Koe, J. Watanabe, T. Sato, and A. Teramoto, Silicon Chem. 1, 67 (2002).

32. For optically active poly(alkylarylsilane)s,

a) D. Terunuma, K. Nagumo, N. Kamata, K. Matsuoka, and

H. Kuzuhara, Chem. Lett., 681 (1998).

b) S. Toyoda and M. Fujiki, Chem. Lett., 699 (1999).

c) H. Nakashima, M. Fujiki, and J. R. Koe, Macromolecules, 32, 7707 (1999).

d) D. Terunuma, K. Nagumo, N. Kamata, K. Matsuoka, and H. Kuzuhara, Polym. J., 32, 113 (2000).

e) H. Nakashima, M. Fujiki, J. R. Koe, and M. Motonaga, J. Am. Chem. Soc., 123, 1963 (2001).

f) S. Toyoda and M. Fujiki, Macromolecules, 34, 640 (2001). g) H. Nakashima, J. R. Koe, K. Torimitsu, and M. Fujiki, J. Am. Chem. Soc., 123, 4877 (2001).

h) P. Dellaportas, R. G. Jones, and S. J. Holder, Macromol. Rapid Commun., 23, 99 (2002).

33. For optically active poly(diarylsilane)s,

a) J. R. Koe, M. Fujiki, and H. Nakashima, J. Am. Chem. Soc., 121, 9734 (1999).

b) J. R. Koe, M. Fujiki, M. Motonaga, and H. Nakashima, Chem. Commun., 389 (2000).

c) J. R. Koe, M. Fujiki, M. Motonaga, and H. Nakashima, Macromolecules, 34, 1082 (2001).

34. For optically active poly(dialkoxysilane), J. R. Koe, M. Motonaga, M. Fujiki, and R. West, Macromolecules, 34, 706 (2001).

35. For a review and books of optically active polysilanes,

a) M. Fujiki, Macromol. Rapid Commun., 22, 539 (2001).

b) M. Fujiki and J. R. Koe, in "Silicon-Containing Polymers: The Science and Technology of Their Synthesis and Applications", R. G. Jones, W. Ando, and J. Chojnowski, Ed., Kluwer Academic Publisher, Dordrecht, NL, 2000, chapt. 24.

c) J. R. Koe, M. Fujiki, H. Nakashima, and M. Motonaga, in "Synthetic Macromolecules with Higher Structural Order", I. M. Khan, Ed. ACS Advance in Chemistry Series No. 812, US (2002, March, Oxford).

36. For reviews of electronic structure of Si-based material.

a) K. Takeda, J. Phys. Soc. Jpn., Suppl. B, 1 (1994).

b) K. Takeda and K. Shiraishi, Comments Cond. Mat. Phys., 18, 91 (1997).

c) N. Matsumoto, Jpn. J. Appl. Phys., 37, 5425 (1998).

37. a) S. Abe, J. Phys. Soc. Jpn., 58, 62 (1989).

b) H. Tachibana, Y. Kawabata, S. Koshihara, T. Arima, Y. Moritomo, and Y. Tokura, Phys. Rev., B, Condens. Matter, 44, 5487 (1991). 
c) S. Abe, M. Schreiber, W. P. Su, and J. Yu, Phys. Rev., B, Condens. Matter, 45, 9432 (1992).

d) R. Nötzel and K. H. Ploog, Int. J. Mod. Phys. B, 7, 2743 (1993).

e) S. Glutsch and F. Bechstedt, Phys. Rev., B, Condens. Matter, 47, 4315 (1993).

f) M. Schreiber and S. Abe, Synth. Met., 55, 50 (1993).

g) X.-F. He, Phys. Rev., B, Condens. Matter, 43, 2063 (1991).

38. a) K. Sakamoto, K. Obata, H. Hirata, M. Nakajima, and H. Sakurai, J. Am. Chem. Soc., 111, 7461 (1989).

b) K. Sakamoto, M. Yoshida, and H. Sakurai, Macromolecules, 23, 4494 (1990).

c) H. Sakurai and M. Yoshida, in "Silicon-Containing Polymers: The Science and Technology of Their Synthesis and Applications", 2nd ed, R. G. Jones, W. Ando, and J. Chojnowski, Ed., Kluwer Academic Publisher, Dordrecht, NL, 2000, chapter 13 .

39. For reviews and books of polysilanes,

a) R. West, J. Organomet. Chem., 300, 327 (1986).

b) R. D. Miller and J. Michl, Chem. Rev., 89, 1359 (1989).

c) J. M. Ziegler and F. W. G. Fearson, Ed., "Silicon-based Polymer Science", Advances in Chemistry Series, 224, American Chemical Society, Washington, D.C., 1990.

d) R. West, in "Comprehensive Organometallic Chemistry II", 2nd ed, E. W. Abel, F. G. A. Stone, and G. Wilkinson, Ed., A. G. Davies, chapter Ed., Pergamon, Oxford, 1995, pp 77-110. e) R. G. Jones and S. J. Holder, in "Silicon-Containing Polymers: The Science and Technology of Their Synthesis and Applications", 2nd ed, R. G. Jones, W. Ando, and J. Chojnowski, Ed., Kluwer Academic Publisher, Dordrecht, NL, 2000, chapt. 12.

f) J. Michl and R. West, in "Silicon-Containing Polymers: The Science and Technology of Their Synthesis and Applications", 2nd ed, R. G. Jones, W. Ando, and J. Chojnowski, Ed., Kluwer Academic Publisher, Dordrecht, NL, 2000, chapt. 18.

40. a) S. Yamaguchi and T. Tamao, in "Silicon-Containing Polymers: The Science and Technology of Their Synthesis and Applications", 2nd ed, R. G. Jones, W. Ando, and J. Chojnowski, Ed., Kluwer Academic Publisher, Dordrecht, NL, 2000, chapt. 17.

b) H. Suzuki, S. Hoshino, K. Furukawa, K. Ebata, C-H. Yuan, and I. Bleyl, Polym., Adv. Technol., 11, 460 (2000).

41. a) F. S. Kipping, J. Chem. Soc., 119, 830 (1921).

b) F. S. Kipping, J. Chem. Soc., 125, 2291 (1924).

42. M. Fujino and H. Isaka, J. Chem. Soc., Chem. Commun. 466 (1989).

43. a) M. Sisido, S. Egusa, A. Okamoto, and Y. Imanishi, J. Am. Chem. Soc., 105, 3351 (1983).

b) J. P. Riehl and F. S. Richrdson, Chem. Rev., 86, 1 (1986). c) S. H. Chen, D. Katsis, A. W. Schmid, J. C. Mastrangelo, T. Tsutsui, and T. N. Blanton, Nature, 397, 506 (1999).

d) H. P. J. M. Dekkers, in "Circular Dichroism: Principles and Applications", 2nd ed, N. Berova, K. Nakanishi, and R. W. Woody, Ed., Wiley-VCH, New York, N.Y., 2000, chapt. 7.

44. a) N. Harada and K. Nakanishi, Ed., "Circular Dichroic Spectroscopy: Exciton Coupling in Organic Chemistry", University Science Books, Oxford, 1983.

b) N. Berova and K. Nakanishi, in "Circular Dichroism: Principles and Applications", 2nd ed, N. Berova, K. Nakanishi, and R. W. Woody, Ed., Wiley-VCH, New York, N.Y., 2000, chapt. 12.

45. a) R. Zink, T. F. Magnera, and J. Michl, J. Phys. Chem. A, 104, 3829 (2000).

b) C.-H. Ottosson and J. Michl, J. Phys. Chem. A, 104, 3367 (2000).

c) J. Michl and R. West, Acc. Chem. Res., 33, 821 (2000).

d) J. Michl and R. West, in "Silicon-Containing Polymers: The Science and Technology of Their Synthesis and Applications", 2nd ed, R. G. Jones, W. Ando, and J. Chojnowski, Ed., Kluwer Academic Publisher, Dordrecht, NL, 2000, chapt. 18.

46. M. Fujiki, J. Am. Chem. Soc., 118, 7424 (1996).

47. P. A. Lovell, "Comprehensive Polymer Science", C. Booth and C. Price, eds, Pergamon, Oxford, 1989, Vol. 1, chapt. 9.

48. a) H. G. Hansma, J. Vesenka, C. Siegerist, G. Kelderman, H. Morrett, R. L. Sinsheimer, V. Elings, C. Bustamante, and P. K. Hansma, Science, 256, 1180 (1992).

b) B. Samori, C. Nigro, A. Gordano, I. Muzzalupo, and C. Quagliariello, Angew. Chem. Int. Ed. Engl., 35, 529 (1996).

c) J. Kumaki, Y. Nishikawa, and T. Hashimoto, J. Am. Chem. Soc., 118, 33213 (1996).

d) U. B. Steiner, M. Rehahn, W. R. Caseri, and U. W. Suter, Macromolecules, 27, 1983 (1994).

e) K. Shinohara, S. Yasuda, G. Kato, M. Fujita, and H. Shigekawa, J. Am. Chem. Soc., 123, 3619 (2001).

49. a) K. Ebihara, S. Koshihara, M. Yoshimoto, T. Maeda, T. Ohnishi, H. Koinuma, and M. Fujiki, Jpn. J. Appl. Phys., 36, L1211 (1997).

b) K. Ebata, K. Furukawa, and N. Matsumoto, J. Am. Chem. Soc., 120, 7367 (1998).

c) K. Ebata, K. Furukawa, N. Matsumoto, and M. Fujiki, Polym. Prepr. (Am. Chem. Soc., Div. Polym. Sci.), 40(2), 157 (1999).

d) K. Furukawa, K. Ebata, and M. Fujiki, Adv. Mater, 12, 1033 (2000).

e) K. Furukawa and K. Ebata, Appl. Phys. Lett., 77, 4289 (2000).

50. a) K. Mislow and P. Bickart, Isr. J. Chem., 15, 1 (1977).

b) P. W. Fowler, Nature, 360, 626 (1992).

c) Z. Zabrodsky, S. Peleg, and D. Avnir, J. Am. Chem. Soc., 115, 8278 (1993).

51. H. Teramae and K. Takeda, J. Am. Chem. Soc., 111, 1281 (1989).

52. a) F. M. Pohl and T. M. Jovin, J. Mol. Biol., 67, 375 (1972).

b) F. M. Pohl, Nature, 260, 365 (1976).

53. E. M. Bradbury, B. G. Carpenter, and H. Goldman, Biopolymers, 6, 837 (1968).

54. a) H. Toriumi, N. Saso, Y. Yasumoto, S. Sasaki, and I. Uematsu, Polym. J., 11, 977 (1979).

b) J. Watanabe, S. Okamoto, and A. Abe, Liq. Cryst., 15, 259 (1993).

c) J. Watanabe, S. Okamoto, K. Satoh, K. Sakajiri, H. Furuya, and A. Abe, Macromolecules, 29, 7084 (1996).

d) A. Abe, H. Furuya, and S. Okamoto, Biopolymers, 43, 405 (1997).

e) K. Sakajiri, K. Satoh, S. Kawauchi, and J. Watanabe, J. Mol. Struct., 476, 1 (1999).

55. Y. Okamoto, T. Nakano, E. Ono, and K. Hatada, Chem. Lett., 525 (1991). 
56. S. Mahadevan and M. Palaniandavar, Chem. Commun., 2547 (1996).

57. Unpublished result.

58. a) E. K. Wilson, Chem. Eng. News (Nov. 6), 35 (2000).

b) R. Feynman, Science, 254, 1300 (1991).

c) K. E. Drexler, Ed., "Nanosystems: Molecular Machinery, Manufacturing and Computing", JohnWiley \& Sons, Inc., New York, N.Y., 1992.

59. For examples,

a) B. L. Feringa, W. F. Jager, B. de Lange, and E. W. Meijer, J. Am. Chem. Soc., 113, 5468 (1991).

b) H. Murakami, A. Kawabuchi, K. Kotoo, M. Kunitake, and N. Nakashima, J. Am. Chem. Soc., 119, 7605 (1997).

c) T. Yamaguchi, K. Uchida, and M. Irie, J. Am. Chem. Soc., 119, 6066 (1997).

d) S. Zahn and J. W. Canary, Angew. Chem. Int. Ed., 37, 305 (1998).

e) K. S. Burnham and G. B. Schuster, J. Am. Chem. Soc., 121, 10245 (1999).

f) C. Mao, W. Sun, Z. Shen, and N. D. Seeman, Nature, 397, 144 (1999).

g) N. Koumura, R. W. Zijistra, R. A. van Delden, N. Harada, and B. L. Feringa, Nature, 401, 152 (1999).

h) T. R. Kelly, H. De Silva, and R. A. Silva, Nature, 397, 150 (1999).

i) R. Schmieder, G. Hübner, C. Seel, and F. Vögtle, Angew. Chem. Int. Ed., 38, 3528 (1999).

j) H. Engelkamp, R. Middelbeek, and R. J. M. Nolte, Science, 284, 785 (1999).

60. a) S. Lloyd, Sci. Am., 273(4), 140 (1995).

b) N. Gershenfeld and I. L. Chuang, Sci. Am., 278(6), 66 (1998).

c) T. H. Oosterkamp, T. Fujisawa, W. G. van der Wiel, K. Ishibashi, R. V. Hijman, S. Tarucha, and L. P. Kouwenhoven, Nature, 395, 873 (1999).

d) Y. Nakamura, Y.-A. Pashkin, and J. S. Tsai, Nature, 398, 786 (1999).

61. a) J. A. Cina and R. A. Harris, J. Chem. Phys., 100, 2531 (1994).

b) R. A. Harris, Chem. Phys. Lett., 223, 250 (1994).

c) J. A. Cina and R. A. Harris, Science, 267, 832 (1995).

d) R. A. Harris and L. Stodolsky, Phys. Lett., Sect. B, 78, 313 (1978).

62. D. H. Rouvray, Sci. Am., 255(3), 36 (1986).

63. a) F. Hund, Z. Physik, 43, 805 (1927).

b) R. Janoschek, in "Chirality-From Weak Bosons to the $\alpha$-Helix”, R. Janoschek, Ed., Springer-Verlag GmbH\&Co., Berlin, 1991, chapt. 2.

64. a) J. Watanabe, Y. Fukuda, R. Gehani, and I. Uematsu, Macromolecules, 17, 1004 (1984).

b) J. Watanabe, H. Ono, A. Abe, and I. Uematsu, Macromolecules, 18, 2141 (1985).

c) H. Kosho, Y. Tanaka, S. Ichizuka, S. Kawauchi, and J. Watanabe, Polym. J., 31, 199 (1999).

d) J. Watanabe, in "Ordering in Macromolecular Systems", A. Teramoto, M. Kobayashi, and T. Norisue, Ed., SpringerVerlag GmbH\&Co., Berlin, Heidelberg, 1994, pp 99-108.

65. a) K. Takeda, H. Teramae, and N. Matsumoto, J. Am. Chem. Soc., 108, 8186 (1986). b) L. A. Harrah and J. M. Zeigler, Macromolecules, 20, 601 (1987).

c) M. Kakimoto, H. Ueno, H. Kojima, Y. Yamaguchi, and A. Nishimura, J. Polym. Sci., Part A: Polym. Chem., 34, 2753 (1996).

d) T. J. Cleij, J. K. King, and L. W. Jenneskens, Macromolecules, 33, 89 (2000).

e) H. Nakashima and M. Fujiki, Macromolecules, 34, 7558 (2001).

66. a) R. West, J. Organomet. Chem., 300, 327 (1986).

b) G. Yenca, Y. L. Chen, and K. Matyjaszewski, Polym. Prepr.

(Am. Chem. Soc. Div. Polym. Sci.), 28(2), 222 (1987).

c) J. P. Banovetz, Y.-L. Hsiao, and R. M. Waymouth, J. Am. Chem. Soc., 115, 2540 (1993).

d) Y.-L. Hsiao and R. M. Waymouth, J. Am. Chem. Soc., 116, 9779 (1994).

67. U. Herzog and R. West, Macromolecules, 32, 2210 (1999).

68. K. Matyjaszewski, Makromol. Chem., Macromol. Symp., 42/43, 269 (1991).

69. J. R. Koe, D. R. Powell, S. Hayase, J. J. Buffy, and R. West, Angew. Chem., 37, 1441 (1998).

70. a) Y. Okamoto, S. Honda, I. Okamoto, H. Yuki, S. Murata, R. Noyori, and H. Takaya, J. Am. Chem. Soc., 103, 6971 (1981). b) W. H. Pirkle and T. C. Pochapsky, Chem. Rev., 89, 347 (1989).

c) G. Blaschke, J. Liq. Chromatogr., 9, 341 (1986).

d) E. Yashima, E. Kasashima, and Y. Okamoto, Chirality, 9, 63 (1997).

e) Y. Okamoto and E. Yashima, Angew. Chem. Int. Ed. Engl., 37, 1020 (1998).

71. a) H. Motoyama, K. Takeda, and K. Shiraishi, Mat. Res. Soc. Symp. Proc., 486, 385 (1998).

b) T. Endo, Y. Sugimoto, K. Takeda, and K. Shiraishi, Synth. Met., 98, 161 (1999).

c) K. Takeda and K. Shiraishi, Solid State Commun., 85, 301 (1993).

72. E. Fossum and K. Matyjaszewski, Macromolecules, 28, 1618 (1995).

73. L. A. Harrah and J. M. Zeigler, Macromolecules, 20, 601 (1987).

74. H. Nakashima and M. Fujiki, Macromolecules, 34, 7558 (2001).

75. a) J.-M. Guenet, H. S. Jeon, C. Khatri, S. K. Jha, N. P. Balsara, M. M. Green, A. Brûlet, and A. Thierry, Macromolecules, 30, 4590 (1997).

b) S. Yue, G. C. Berry, and M. M. Green, Macromolecules, 29, 6175 (1996).

76. a) R. D. Miller and R. Sooriyakumaran, J. Polym. Sci., Polym. Lett. Ed., 25, 321 (1987).

b) R. D. Miller and R. Sooriyakumaran, Macromolecules, 21, 3122 (1988).

c) P. Cotts, R. D. Miller, and R. Sooriyakumaran, in "SiliconBased Polymer Science", J. M. Zeigler and F. W. G., Fearon, Ed., American Chemical Society, Washington D.C., 1990, chapt. 23, p 397.

d) J. Michl, Synth. Met., 49, 367 (1992).

77. a) Y. E. Ovchinnikov, V. E. Shklover, Y. T. Struchkov, V. V. Dement'ev, T. M. Frunze, and B. A. Antipova, J. Organomet. Chem., 335, 157 (1987). 
b) Y. E. Ovchinnikov, V. V. Dement'ev, V. E. Shklover, Y. T. Struchkov, T. M. Frunze, B. A. Antipova, and V. A. Igonin, Makromol. Chem., 190, 3195 (1989).

78. Unpublished work. Due to the noisiness of the CPPL signal, it was difficult to accurately ascertain the absolute intensity.

79. J. J. L. M. Cornelissen, M. Fischer, N. A. J. M. Sommerdijk, and R. J. M. Nolte, Science, 280, 1427 (1998).

80. a) S. J. Holder, R. C. Hiorns, N. A. J. M. Sommerdijk, S. J. Williams, R. G. Jones, and R. J. M. Nolte, Chem. Commun., 1443 (1998) b) N. A. J. M. Sommerdijk, S. J. Holder, R. C. Hiorns, R. G. Jones, and R. J. M. Nolte, Macromolecules, 33, 8289 (2000).

81. a) H. Tachibana, H. Kishida, and Y. Tokura, Langmuir, 17, 437 (2001).

b) H. Tachibana, H. Kishida, and Y. Tokura, Appl. Phys. Lett., 77, 2443 (2000).

c) M. Yoshida, M. Mori, S. Yokokawa, F. Nakanishi, and H. Sakurai, Mol. Cryst. Liq. Cryst., A322, 135 (1998).

d) M. Yoshida, Mol. Cryst. Liq. Cryst., A327, 71 (1999).

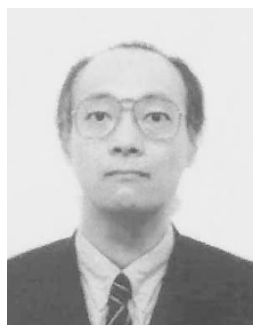

Michiya Fujiki was born in Fukuoka, Japan, in 1954. He received B.S. and M.S. degrees in chemistry of organic synthesis from Kyushu University, Fukuoka, Japan in 1976 and 1978, respectively, and received his Ph.D. degree from Kyushu University, Fukuoka, Japan, in 1993. Since 1978, he has worked for Nippon Telegraph and telephone Cooperation (NTT). From 1978 to 1982, he studied low-loss optical plastic fibers and from 1983 to 1987, he studied the preparation and thin film properties of semiconducting phthalocyanine derivatives. In 1987, he joined the Basic Research laboratories of NTT. From 1987 to 1991, he initiated the study on synthesis and photophysical properties of polysilylenes bearing aryl groups. From 1992 to 2002, he has been engaged in the study of synthesis and photophysical properties of optically active polysilanes and $\pi$-conjugating polymers. Since May 2002, he is a full professor at advanced polymer science laboratory, graduate school of materials science, Nara institute of science and technology (NAIST). Prof. Fujiki is a member of the Society of Polymer Science Japan, the Chemical Society of Japan, the Society of Silicon Chemistry Japan, and the American Chemical Society.

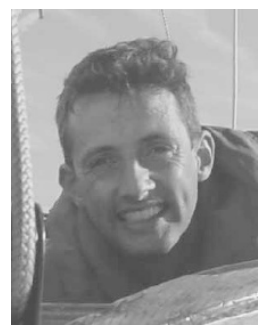

Julian Robert Koe was born in the U.K. in 1964. For his undergraduate study, he read a combined honours degree in Chemistry and Law at Exeter University, graduating in 1986, and then received his Ph.D. degree from the same university in 1989 for research in platinum metal complex chemistry. He was a Japan Society for the Promotion of Science post-doctoral researcher at Tohoku University from 1989-1991 (silylene iron complexes), a Royal Society Return Fellow 1992-1993 (platinum metals chemistry), Toshiba Fellow 1993-1995 (novel alkoxy polysilanes), Research Assistant at the University of Wisconsin 1996-1998 (polysilane and polysiloxane chemistry) and post-doctoral fellow at Nippon Telegraph and Telephone Cooperation (NTT) Basic Research Labs 1998-2000 (chiral polysilanes). He joined the faculty at the International Christian University in Mitaka, Tokyo in 2001 as Assistant Professor in the Department of Chemistry. His current research interests include the study of the chemistry, photophysics, chirality, and fluxionality in small and large molecule silicon chemistry and transition metal chemistry. His favorite pastime is sailing.

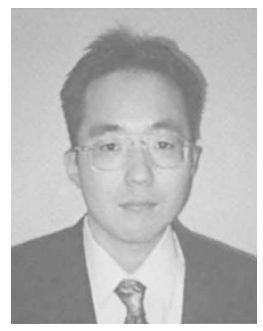

Ken Terao, born in Osaka in 1971, graduated from the Department of Macromolecular Science, Osaka University in 1994, and received his Dr. of Science degree from Osaka University in 1999. He studied solution properties of regular-branched polymers during his doctoral course research. From 1999 to 2001, he worked at Ritsumeikan University for Japan Science and Technology Corporation (JST) as a researcher and studied helical structure of optically active polysilanes in solution. In 2001, he joined the Department of Biological and Chemical Engineering, Gunma University as an Assistant Professor. His research interests include solution properties of polymers and synthesis and properties of microcapsules.

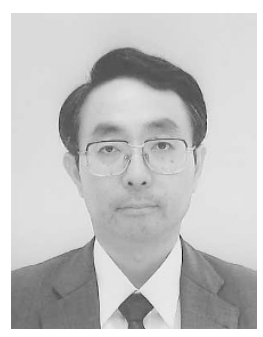

Takahiro Sato was born in Osaka in 1957. He graduated from the Department of Macromolecular Science, Faculty of Science, Osaka University in 1980, and received his Ph.D. from Osaka University in 1985. He started working in 1985 as a guest scientist at the National Bureau of Standards (now the National Institute of Standards and Technology) in the United States, and then joined the Department of Macromolecular Science, Osaka University as a research associate in 1987. He promoted to an Associate Professor in 1996 and a Full Professor in 2002 at Osaka University. His major research field is polymer solution properties, and he is now interested in the interrelation between the polymer interaction and various solution properties, e.g., rheological properties, liquid-crystalline structure, and phase or aggregation behavior. 


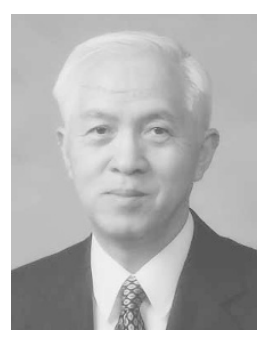

Akio Teramoto, born in 1933 (Ishikawa, Japan), received his B.S. in 1956 and Ph.D. in 1965 (Kyoto Univ.). He worked at Osaka University from 1961 to 1997, where he is Professor Emeritus, and is currently Chair Professor at Ritsumeikan University and research staff of CREST of Japan Science and Technology Corporation. He received The Award of the Society of Polymer Science, Japan (1974) and SPSJ Award for Outstanding Achievement in Polymer Science and Technology (1995). His research interests includes chain conformations and conformational transitions in dilute solution of polypeptides, polyisocyanates, polysaccharides, and polysilylenes, thermodynamics and dynamics of stiff polymer solutions including polymer liquid crystals, and water structures in aqueous biopolymer solutions.

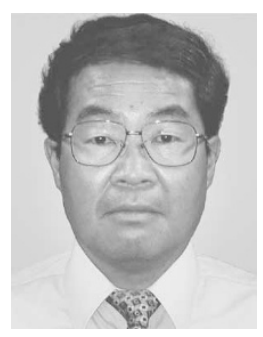

Junji Watanabe, born in 1948 (Ehime, Japan), received his B.S. in 1970 and Ph.D. in 1976 (Tokyo Institute of Technology). Since 1976, he worked as research associate, assistant professor, and full professor at Department of Polymer Chemistry in Tokyo Institute of Technology. His research interest is the structure and properties of polymer liquid crystals based on polypeptides, polysaccharides, polysilanes, and polyesters. 